\section{LA-8488-T}

Thesis
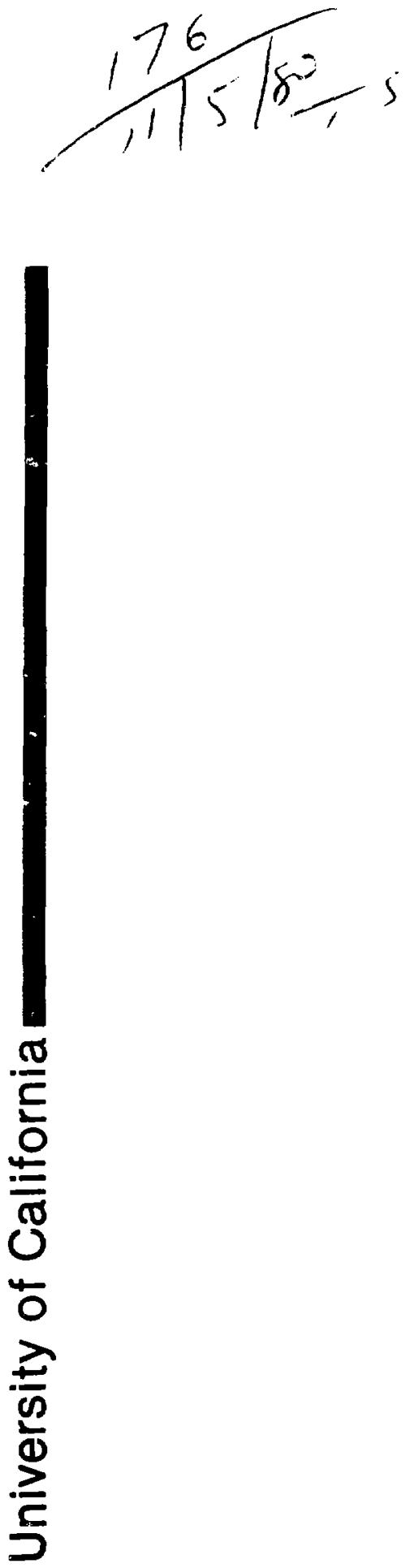

Theory of the Arc Discharge in Air Blast Breakers

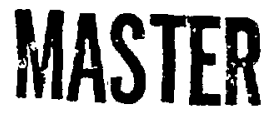

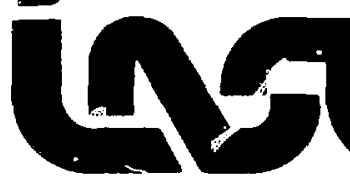




\title{
Theory of the Arc Discharge in Air Blast Breakers
}

\author{
Herbert Friedrich Vogel
}

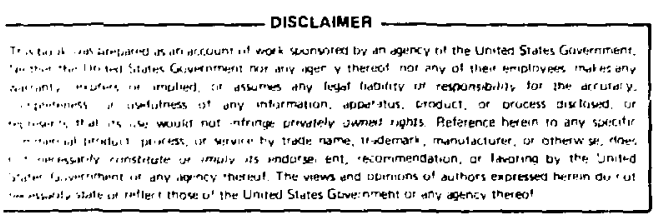


CHAPTER

1. INTRODUCTION

1.1 State of the Art 4

1.2 Scientific Motivation 8

1.3 Objectives 8

1.4 Method and Conventions 10

2. CHARACTEKIZATION OF ARC PLASMA 14

3. HYDRODYNAMIC EQUATIONS OF MILTICOMPONENT PLASMAS 18

3. J Introduction 18

3. 2 Selection of the Solution Method 19

3. 3 The Multicomponent Plasma 21

3.3.1. Multicomponent Averages 21

3.3.2. Derivation of the Maxwe11 Equation etc. 22

3.3.3. The Moment Equations 28

4. SAHA'S EqUATION FOR A THREE CONiPONENT NONISOTHERMAL PLASMa 32

5. ELASTIC COLIISIONS 40

6. CYLINIRICAL ARC ENVELOPE FUNCTION 43

7. CURRENT EQUATION AND SUMMARX OF EQUATIONS 49

7.1 Current Equation 50

7.2 Energy Flow Equation 55

7.3 Summary of Equations 56

8. STATIONARY CONDITIONS 60

9. LAGRANGIAN FUNCTION 61

9.1 Interaction Free Energy (particle-particle) 61

9. 2 Field Free Energy (particle-external field) 65

10. RADIATIVE ENERGY TRANSPORT 68

11. HEAT CONDUCTIVITY 69

11.1 Atomic Nitrogen $\quad 69$

11.2 Thermal Conductivity of the Lorentz Gas 70

11.3 Enhancement of Thermal Conduction by Magnetohydrodynamic Turbulence $\quad 70$ 
12. TURBULENCE

13. ClOSURE AND STUDY OF THE STEADY STATE 77

14. SOME CONSIDERATIONS FUR THE TRANSIENT STATE

15. CONCLUSIONS 84

16. SUMMAKY 87

APPENDIX

A. CUNVERSIUN FROM CGS TU MKS UNITS

B. ENSKOG-CHAPHAN METHOD FUR SOLVING THË BOLTZMANN AND
MAXWELL TRANSPORT EQUATIUNS

1. Outline of the Method 91

2. The Zeroth Urder Approximation 98

3. The First Order Correction 99

4. Calculation of the Quantities A and B 108

5. Scattering Cross Section 113

$\begin{array}{ll}\text { C. LIOUVILLE'S EQUATION } & 120\end{array}$

ACKNOWLEDGIENTS $\quad 122$

REFERENCES 123 
THEORY OF THE ARC DISCHARGE

IN AIR BLAST BREAKERS

Herbert Friedrich Vogel, Ph.D.

\begin{abstract}
We study the airblast breaker, ncted for its large current interrupring capacity. Our theoretical model is for the so-called double jet configuration in which a quasicylindrical arc develops during current interruption between the parting electrodes. The arc is cooled by a jet stream of air expanding from a pressurized reservoir, flowing radially inward in the space opening between the parting electrodes, and finally ejecting axially through the center bore of the electrodes.

In developing arc plasma models, recourse is generally taken to heuristic formulations, entirely on the macroscopic level. There is one notable exception, in which a study of the hydrodyamic aspects is reported in an application of a 13 moment metnod for approximating
\end{abstract} Boltzmann's kinetic equation.

Our own formalism is based on the normal Enskog-Chapman approximation in deriving the hydrodynamic equations from the Boltzmann equation. This method is much simpler to present than a 13 moment metnod. Because single charged positive nitrogen ions and atoms, in addition tu electrons, are the predominant species, we consider such a three-component plasma. We use a reported simple parameterization of the heat transport by turbulent air and study the are plasma's evolution, from the end of one lelaxation time interval.

We show that the Boltzmann approximation applies and the arc plasma is in thermodynamic equilibrium, with characteristic imes of less than a few tens of nanoseconds. We minimize the Helmholtz free energy on the microscopic and macroscopic scales to determine the arc's stable equjlibrium and obtain relationships for closing our system of hydrodynamic equations. Using reported experimental values of pressure and air velocity to test our equations, we calculate the voltage 
gradient, arc radius, and temperature. Our calculations agree with observation, when we use two fitting parameters. Those are the flow density of the surface heat dissipated by the turbulent air and the opacity. Our results indicate those two as being the dominant heat transport mechanisms.

The radial cumponent in the temperature gradient is negligible in the arc's interior, because of the high degree of transparency and the magnetohydrodynamic $m=0$ turbulence. In thermodynamic equilibrium, the $m=0$ turbulence is shown to evolve on the time scale of one half to a jew microseconds. Because the external gas pressure exceeds the electromagnetic pressure by at least one order of magnitude, the arc is wall confined. tiessure gradients deriving from intense surface couling can cause the wall confinement to become upsat when the Kadomtsev stability criterion is locally no longer satisfied, the $m=0$ instability this resulting. The set of equations obtaining in the arc's length element is thus developed, taking into account the significant boundary effects. The arc length is determined by the evolution of the state variables in the external circuit equations, closed by our relationships for the radius and voltage gradient, and an expression for the inductance variation as a function of radius and length.

We minimize the Helmholtz free energy on the microscopic and macroscopic scales to obtain relationships for closing our system of differential equations. Using the reported experimental fressure and air velocity vs the axial coordinate to test our equations, we calculate the voltage gradient, arc radius and temperature. Our calculations evidently agree with observation when we use two ficting parameters. These are the heat flow parameter for the turbulent transport, and the opacity of gray body radiation. Our results seem to indicate that these two are the dominant heat transport mechanisms.

Regarding the microscopic basis for our formalism, we show that the Boltzmann approximation is applicable to provide us with a microscopic picture of the arc plasma. We snow that the stationary condition is asymptotically stablə, with the characteristic times of typically less than a few tens of nanoseconds. 
In summary, the complete set of equations obtaining in the arc's length element are given. The arc length is determined when the external circuit equations are closed by an expression for the arc inductance as a function of the radius and length, in addition to our relationships for the adius and voltage gradients. 


\section{CHAPTEK 1}

INTRODUCTIUN

\subsection{State of the Art}

The electric arc is one of the phenomena of great importance in the operation of practical electrical sys'ems. Yet, its underlying physical mechanisms are still mystery to most engineers. That observation is taken verbatim from the two pertinent texts, that is W. Riede:, Plasma and Arc (1967, in German), and T. H. Lee, Physics and Enginering of High Power Switching Devices (1975). The two authors make a laudable attempt to introduce some of the important physics background to an engineering audience. A well written overview is offered in Chap. 3 of the book of A. Erk and M. Schmelzle, Elementary Switching Iechnology (1974, in German) which is worth reading, partly because it is free of the many inaccuracies encountered in the other texts. No closed mathematical form seems to be presented in any of the material. mentioned thus far. Not surprisingly, therefore, A. Grütz is led to conciute in his doctoral dissertation on A Computer Study of Circuit Breakers, Using a Generalized Arc Theory (Technical University Aachen, 1970) that $a)$ it is impossible to make an adequate estimate of the current interrupting capacity of a given circuit breaker interacting witr a given circuit; b) the microscopic processes elude mathematical analysis for reasons of their complexity and multitude; and c) a useful heuristic method is developed, not considering the microscopic processes. We mention all this because of the great significance which the empirical methods have gained over the years, while the microscopic processes have consistently been regarded as elusive of mathematical treatment. From a practical point of view, that emphasis of the heuristic methods is not necessarily a disadvantage, because one is interested primarily il: the resistance changes as a function of time, as in the electric spark, when the voltage between the electrodes breaks dokn. When air and airblast circuit breakers are concerned, one can take the presence of hot arc spots for granted as the source of an ample supply of charge carriers. The arc resistance follows, thus, from 
certain empirical assumptions on the arc column radius, length, and temperature, which are the bases of the frequently cited arc models such as those of 0 . Mayr, A. M. Cassie, and other more refined ones.

In a way, one might compare this state of affairs with that in thermodynamics of times past, before the Austriar. physicist Ludwig Boltzmann had published the first microscopic, i.e., kinetic, equation in 1872. James Clerk Maxwell had published his macroscopic, i.e., hydrodynamic, equation a few years earlier, immediately leading to the fluid dynamic equations of change as they had been known at the time (Euler, Navier, Stokes), as well as to those developed later (Burnett). Thus, the two greatest physicists of the century developed the mathematical relationships between the microscopic world of Hamilcon's equations of motion, governirg the trajectories of gas molecules, and the macroscopic fluid dynamic equations. These are known as Euler's equations and, to a higher order, the Navier-Stokes and Burnett equations. Realizing the complete hopelessness ever to integrate the set of equations of motion of the cosmological number cf nolecules contained in a practical volume, Boltzmann developed the statistical formalism by which the unique equivalence between Hamilton's and Boltzmann's equations is established, and which exhibits the important Eurther property of time irreversibility. Thus, the Boltzmann equation defines the evolution of the statistically defined quantity called the distribution function which tends toward the equilibrium of maximum randomess and entropy, according to the equation, which was expected from thermodynamics. The equilibrium distribution is known as the Maxwe11-Boltzmann velocity distribution which has the form of a Gaussian distribution of molecular speeds. The microscopically conserved quantitles, called the dynamical functions, are averaged against that distribution function (whether equilibrium or not). Maxwell's hydrodynamic equation is a differential equation relating the velocity-averaged dynamical functions to the configuration space and time. The microscopically conserved quantities are the mass, momentum, and energy. The distribution function is defined as a number density in a six-dimensional space similar to the phase space in mechanics. Such a 
six-dimensional volume is shown, generaily, to be conserved in an ensemble or "cloud" of molecules evolving in that space, with gains and losses in the ensemble's 6-volume being caused by the collective effects of collisions. Because of the collisional gain versus loss integral, the Boltzmann equation is nonlinear and its integral is obtainable, in its exact form, only in two special cases. One is the noncollisional limit, which exists in the incompressible fluid. The other is that of the Maxwell Molecules for which Maxwell could derive the Boltzmann equation from his hydrodynamic equation. Maxwell Molecules are hypothetical, interacting according to the special force law, $F \propto r^{-5}$, where $r$ is the separation between two isolated molecules. The difficulty in the case of the electric arc, and plasma physics in general, lies in the Coulomb interactions, with their force law $F \times r^{-2}$, for which the kinetic equations are difficult to integrate.

Much of the matters discussed, thus far, is generally familiar from undergraduate studies at the level of Reif, Fundamentals of Statistical Physics and Thermal Physics, which are a part of most physics, mechanical, and chemical engineering curricula. The more challenging problems in the electric arc lie in the selection of an appropriate kinetic equation, determining whether there is statistical equilibrium, finding an appropriate integrating formalism, and closing the system of equations, considering the boundary conditions. In summary, the state of the art may be described by a variety of heuristic equations, as contrasted by a lack of closed systems of integrated kinetic equations. One important exception exists in the latter category, namely the work of Suchy and Thiel, in which the Boltzmann Equation is integrated, as discussed in the paragraph immediately following.

Instead of integrating both sides of the Boltzmann Equation, one can derive the integral of the left hand side (l.h.s.), which is the set of terms called the streaming function and take for the collision integral [that is the right hand side (r.h.s.)] the collision frequency formulas. The latter are known for certain idealizations, such as the fully ionized plasma. Formulas of that kind are related to the Spitzer 
resistivity and thermal conductivity, published in many plasma physics texts. One part of our work is to develop the $1 . \mathrm{h} . \mathrm{s}$. , done in Chaps. 3 and 7. Another part is to develop the integral of the r.h.s., done in App. B, and compare our results for the fully ionized plasma with the Spitzer formulas. In extensive papers, Grad (1949), Weitzsch, and Suchy (1964) have reported a formalism known as the Thirteen Moment Method: The distribution function is expanded in a complete set of orthogonal functions, in the velocity, with coefficients $a_{m}(\underset{r}{,} t)$, which are functions of the spatial coordinate $\underline{r}$ and time. The Maxwe11-Boltzmann distribution is contained as a component in the expansion. By substituting the expansion in the Boltzmann equation, one can obtain an infinite set of nonlinear differential equations for $a_{m}$. The various

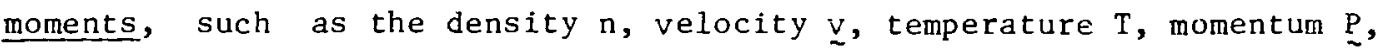
and current density $\mathrm{J}$, are simple functions of the coefficients $a_{m}$. In his dissertation, Thiel applies Suchy's coefficients to the special case of the arc plasma, developing the transport coefficients for it. The Thirteen Moment Method is applicable for the equilibrium and nonequilibrium case.

Let us return to a short introductory discussion of our uwn formalism, relating it to the state of the art. We show that the arc plasma is in statistical equiliorium. We can use, therefore, the normal solution of Enskog and Chapman in the hydrodynamic approximation which permits the much simpler presentation in our App. B, when compared with the more complicated Thirteen Moment Method. In summary, the distribution function is expanded in the uniformicy parameter $\varepsilon$, measuring the departure of the state from that of local equilibrium, $\mathrm{f}=\mathrm{f}(0)+\varepsilon f(1)+\cdots$. Near the local equilibrium, one can define the number density $n(\underset{\sim}{r}, t), f l u x ~ n \underset{\sim}{v}(\underline{r}, t)$, and energy density $3 / 2 n k T(\underline{r}, t)$, as slowly varying functions of their arguments $(\underset{r}{r}, t)$ and show that $\varepsilon$ is proportional to the spatial gradient of tnose quantities. We show the system of differential equations developed in Chaps. 3 and 7 can be closed, which we do by minimizing the free energy defined in thermodynamics. 


\subsection{Scientific Motivation}

The microscoplc processes are generally considered to be beyond the nathernatical scope of a reasonable arc model. Ohe takes recourse to heuristic: formulations, entirely on the macroscopic level. Celebrated are the early empirical models of A. M. Cassie (1939), and 0. Mayr (1943). A noted exception is the important work by H. G. Thiel (1970), i which is a thorough study of the hydrodynamic aspects and an application of Suchy's 13-moment method for solving the Boltzmann kinetic equation. Aside from this particular application, use of the Weitzsch-Suchy methc. ${ }^{\star}$ scems uncomnon, at least in the English language literature. ${ }^{2}$ Yet, familiarity with Suchy's extensive treatise ${ }^{3}$ is necessary if one is intent on penptrating Thiel's work. However, deviations from statistical equilibrium are small in the electric arc. It follows that only the zeroth and first order of $i$ are needed in the Enskog-Chapman approximation, which is far more amenable to being presented in a transparenc and compact formo by closing the equations, considering boundary and energy conditions, we can fill in another gap in the present literature.

\subsection{Dbjectives}

As in Thiel's dissertation, our focus is on the air blast breaker, noted for its encrmous interrupting capacity and the resulting popularity among users in the electric utility industry, second only to the $\mathrm{SF}_{6}$ breaker. Because of the large number of ionized species present in the $\mathrm{SF}_{6}$ arc, we choosa the air blast breaker, being mors tractable as a three component plasma device. The configuration of the parting electrodes causes a quasi-cylindrical arc to evolve, as shown in the three configurations of Fig. la-c. An actuating mechanism drives the electrodes. The arc is cooled by a jet stream of air expanding with

\footnotetext{
*Weitzsch was probably first in extending Grad's well known 13-moment method mentioned in Sec. 3.2 to pronounced nonequilibrium. Suchy's paper offers a thorough analysis of the 13 -moment methods.
} 

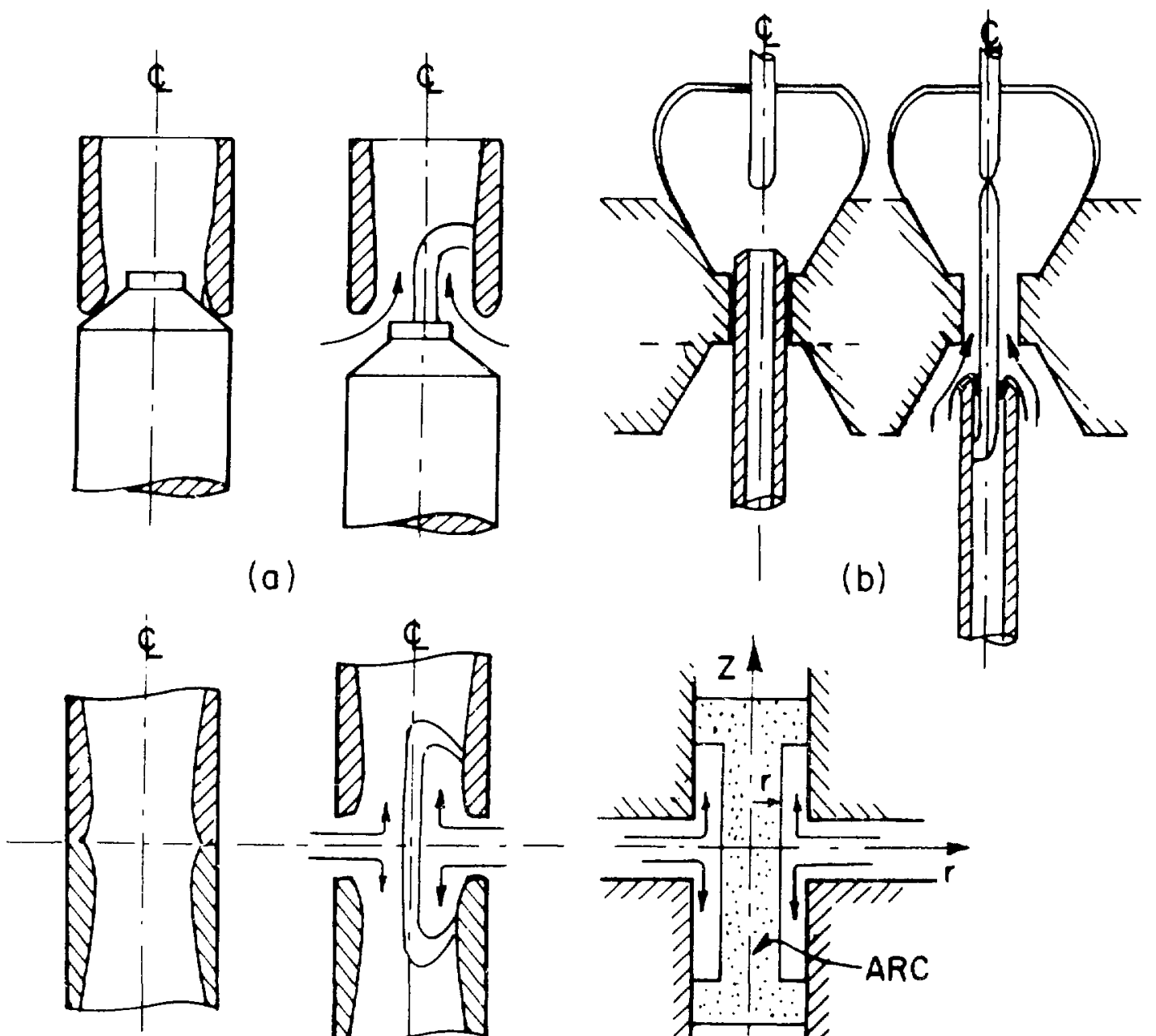

(a)

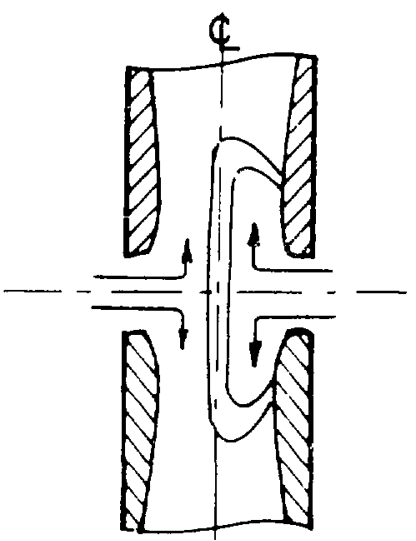

(c)

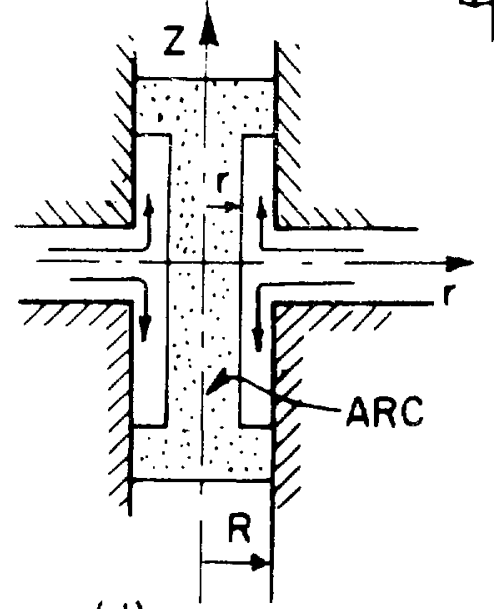

(d)

Fig. 1 Contacts in air blast breakers.

a) Single jet contacts

b) Single jet contacts with one tubing type contact

c) Double jet contacts

d) Idealization of double jet contacts, for model 
nearly sonic velocity from a pressurized reservoir into the atmosphere. The arrangement in Fig. Ic is symmetrical about the midplane, with the compressed air streaming radially lnward, forming a stagnation point in the center, and ejecting axially through the center bore of the electrodes. The reservoir pressure is approximately 30 to 50 bar in a modern airblast breaker.

It is our objective to derive, for the configuracion of Fig. 1c, a set of equations from first principles and in a well understood formalism and then, to simplify the equations as drastically as possible, still yielding agreement with known observation. For the stationary arc, our results are compared with observation.

Because the voltage drop at the electrode spots is negligible when compared with that in the arc column, we neglect all electrode voltage drops. The great magnitude of the arc drop in compressed air is indeed one of the air blast breaker's attractive features. It serves in the field to limit the current in many applications. We shall not consider afterglow phenomena.

\subsection{Method and Conventions}

From Fig. 1c we get, by idealization, tie axisymmetric geometry depicted in Fig. Id, where we assume the arc radius $*$ to be fairly constant along the arc's center portion. Our idealization of neglecting the electrode drop is indicated schematically in Fig. ld, by the two fictitious disks of arc plasma.

We discuss the Boltzmann equation. Its application is justified in our plasma parameters. We develop the hydrodynamic equations, using the normal solution method for the collision integral. Because singly charged positive ions and neutral atoms, in addition to the electrons, are the predominant species, we are considering such a three component plasma. We use Thiel's parameterization of the heat transport by turbulent air.

Because of the prevailing air pressure being at least atmospheric in arc chambers, the attendant electron densities are such as to imply Debye lengths of 0.5 to $2.5 \mu \mathrm{s}$, and relaxation times of 
subnanosecond duration. One of the important criteria for the validity of the Boltzmann equation is thus satisfied in our application, i.e., the existence of a time interval $\Delta t$, long compared with the collision time ${ }^{\tau_{c}}$, and short compared with the relaxation time $\tau_{r}$, where $\tau_{r}$ is on the order of the mean time interval separating subsequent collisions. We will show in Chap. 2 that ${ }^{\tau_{c}} \ll \Delta t \ll \tau_{r}$ is indeed satisfied. Physically, this condition provides the assurance that the collisions are well defined in space and time, with no overlapping between successive collisions of the same particle. This is an essential prerequisite for the idealization made in Boltzmann's molecular chaos assumption. It permits the treatment by simple binary collisions and evaluation of the loss-gain balance by simply counting collisions. Another important consequerce of this two-body picture is its Markovian character. In other words, the evolution of the quantities of interest, depends only on their instantaneous values and not on previous history--as it would if many body collisions had to be considered. Hence, the system evolves asymptotically as long as it is not far removed from equilibrium. Knowledge of the arc ignition phase is, therefore, redundant to the study of the evolution.

As a final prerequisite ${ }^{4}$ for validity in applying the Boltzmann equation, we fino the Debye length to be small, being certainly several orders of magnitude less than the smallest linear dimension of practical interest in the arc column. Hence, the distribution function does not change appreciably over a range of distances travelled by an average particle in a time interval $\Delta t$. As a further consequence of this condition, the generic properties of the arc are those of a plasma and not an ionized gas. The electron and ion temperatures must therefore be expected to be nearly the same.

Moment equations are not closed. They are the transport equations, i.e., relationships between hydrodynamic variables, leaving us with too many unknown functions. Additional relationships are needed for closure. One equation is created by the assumption that the plasma as a whole be stationary, which means that a stationary state is rapidly attained in which the sums of the electrons and ions possess equal, but 
oppositely direrted, momenta. Another relationship is contributed by the boundary condition that, the total current equal that of the external circult. Two additional relationships are obtained from the requirement of a stationary free energy. The free energy has an extremum for a thermodynamic equilibrium system of fixed volume and mass, i.e., fixed density or specific volume. Entrema are determined with respect to an ionization process and the Coulomb interactions. The former yieids the Saha equation, 1.e., the law of mass action for the chemical equilibrium, and the latter results in two separate conditions, i.e., a microscopic and a macroscopic one. In the microscoptc system, the Debye theory is usually used with the result that each electron and ion is effectively surrounded by a cloud of shieldirg charges with a spatlal distribution function such that the free energy of the Coulomb interaction is an extremum. In the macroscopic system, we gain a relationship between the thermodynamic variables of random and drift velocities, i.e., between the electron temperature and the current. We determine the locus for this condition in Sec. 9.2, and Chap. 13.

We are thus left with only three external quantities, i.e., the gas kinetic pressure, the current, and the external heat dissipation parameter, complemented by our presumed existence of a constant current density profile within a cylindrical arc envelope. We show in Chap. 6 that a cylindrical arc envelope is indeed associated with a constant current density, provided the heat transfer function within the arc column is 1nd. ndent of the radius. We can show, in Sec. 11.3, that the radial ten $\iota_{f}$ ature gradient is indeed negligible, because of magnetohydrodynamic turbulence evolving on the time srale of 0.5 to a few microseconds, under the existing boundary conditions on the arc surface. Consequently, we have a quasi-cylindrical arc envelope and a nearly flat current profile.

In summary, we study the arc's evolution, starting at the end of one relaxation time interval. Because we can close the set of hydrodynamic relationships, we obtain, in the stationary condition, a determined system of algebraic relationships, with characteristic times $\tau \simeq 0.1 \mathrm{~ns} \gg \tau_{\mathrm{r}}$. 
We use Gauss' symmetrical units or mks units throughout. Gaussian units in the case of our equations are identical with the esu. In App. A we show the conversion from cgs to mks. In addition, esu or mks is indicated in front of each chapter.

Only when referring to some $\mathrm{Eq}$. (j) in a different chapter or appendix, $i$, we denote the reference as Eq. $(i-j)$. We omit $i$ in references within the same chapter or appendix, simply saying Eq. (j). 
CHAPTER 2

CHARACTERIZATION OF ARC PLASMA

(esu)

Boltzmann's molezuiar chaos assumption means that the collision frequency can be calculated as long as time intervals at are considered for which the mean flight time $>\Delta t$. > average collision time. In other words, the dynamic relaiions between successive collisions are easily lost in chaos because the scatterers are so numerous and randomly distributed in space. This fundamental assumption imposes a limitation on the validity of many calcuiations in plasma physics, especially when low density plasmas are concerned. It imposes no limitation of consequence on arc plasma calculations. Three types of Coulomb interaction are considered in plasma physics, i.e., the short range, long range, and very long range interactions. In the case 0 : the short range interactions, particles appruach one another with an impact parameter of

$$
b_{0}=e^{2 / 3 k T}
$$

or less, so they will be scattered through large angles, of at least $90^{\circ}$, in any single encounter. The cross section for this type of event is given by $\sigma_{S}=\pi b_{0}^{2}$ and $i t$ is easy to verify that the corresponding collision Erequency is given by

$$
v_{s}=\pi n e^{4} m_{e}^{-1 / 2}\left(3 k T_{e}\right)^{-3 / 2} \text {. }
$$

The long range interactions are limited by the shielding distance

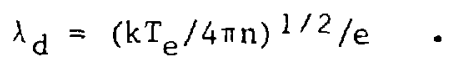

The corresponding interaction time in a single encouncer, $\tau_{c}=\lambda_{d} / \bar{v}$ is 
determined by the distance $\lambda_{d}$ over which particles may interact by their Coulomb fields. Hence, it is easily verified that

$$
i_{c}=\frac{1}{e}\left(\frac{m_{e}}{12 \pi n_{e}}\right)^{1 / 2} .
$$

Rutherford scattering determines the distant collisions between electrons and ions. It is discussed in Chap. 5 and App. B-5. The corresponding collision frequency $\nu_{r} \equiv v_{e i}$ is shown in Table $I$ to be murh greater than the short range collision frequency.

Interactions over the very distant range $i>\lambda_{d}$ do not occur, because of shielding, in a plasma with Maxwellian particle distribution in velocity space, as shown by the Debye-hückel theory. In cases where encounters over the very distant range do have to be considered, the Vlasov equation provides a good model. In Table I we indicate that for extremely small currents, especially if the pressure is relatively small, the relative drift velocity may assume values close to 1 , which would mean the electric field gradient was too great for the theory to be applicable. The energy gained in the electric field would then be comparable with the initial, i.e., the thermal energy. It would then be possible for electrons to accelerate to very high velocities and not be scattered at all, because the Rutherford scattering cross section decreases like (energy) ${ }^{-2}$. This is the phenomenon studied by Giovanelli and nreicer.5,6 our discussion is then inadequate because it is based on small deviations from equilibrium, i.e., on elastic collisions. Observe tha' in some recent arc related work ${ }^{7}$ it seems to be assumed that the drift velocity were of a magnitude comparable with the thermal velocity of the particle.

Finally, we make two important assumptions, i.e.,

a) that the arc plasma is homogeneous, which has been emphasized already; and 
TABLE I

CHARACTERISTIC DATA FOK ARC. PLASMA

\begin{tabular}{|c|c|c|c|}
\hline Gas kinetic pressure & bar & 1 & 24 \\
\hline Equilibrium temperature & $k k$ & 16 & 20 \\
\hline Electron density & $m^{-3}$ & $1.3 .10^{23}$ & $2.6 \cdot 10^{24}$ \\
\hline Neutral particle density & $m^{-3}$ & $1.8 \times 10^{23}$ & $4 \cdot 0 \cdot 10^{24}$ \\
\hline Total current & $h \dot{H}$ & $0.1|1.3| 2.4$ & $1.3 / 2.4$ \\
\hline Current density & $10^{8} \mathrm{Am}^{-2}$ & 1.910 .810 .7 & $1.6 \mid 1.31$ \\
\hline Impact parameter, $90^{\circ}$ scattering $b_{0}$ & $\AA$ & 3.5 & 2.8 \\
\hline Short range scattering cross section $\sigma_{S}$ & $x^{2}$ & 38 & 24 \\
\hline Long range scattering cross secton $o_{d}$ & $\AA^{2}$ & $1.8 \times 10^{5}$ & $1.2 \times 10^{4}$ \\
\hline$\Lambda=r_{d} / r_{s}$ & 1 & 70 & 22 \\
\hline 2. $n \Lambda$ & 1 & $4 \cdot 2$ & 3.1 \\
\hline Colliston (i.e., interaction) time ${ }^{\top} \mathrm{c}$ & ns & $2.8 \times 10^{-5}$ & $0.64 \times 10^{-5}$ \\
\hline Relaxation time ${ }^{T} r$ & ns & $71 \times 10^{-5}$ & $6.7 \times 10^{-5}$ \\
\hline Rat 10 $\tau_{c} / \tau_{r}$ & 1 & $4.0 \times 10^{-2}$ & $9.5 \times 10^{-2}$ \\
\hline No. cf particles in a Debye space & 1 & 7.7 & 2.4 \\
\hline Mean random speed $\bar{v}_{\mathrm{e}}=\left(\frac{8}{\pi} \frac{\mathrm{kT}_{\mathrm{e}}}{\mathrm{m}_{\mathrm{e}}}\right)^{\mathrm{i} / 2}$ & $\mathrm{~m} / \mathrm{s}$ & $8 \times 10^{5}$ & $9 \times 10^{5}$ \\
\hline $\bar{v}_{i}=\left(\frac{8}{\pi} \frac{k T_{i}}{m_{1}}\right)^{1 / 2}$ & $\mathrm{~m} / \mathrm{s}$ & $5 \times 10^{3}$ & $6 \times 10^{3}$ \\
\hline Drift velocity $\mathrm{v}_{\mathrm{de}}=\mathrm{J}_{\mathrm{e}} / \mathrm{en}$ & $\mathrm{m} / \mathrm{s}$ & $9 \times 10^{3} 14 \times 10^{2} 13 \times 10^{3}$ & $350 / 400$ \\
\hline$v_{d i}=J_{i} / e n$ & $\mathrm{~m} / \mathrm{s}$ & $20 / 50$ & $2 / 5$ \\
\hline Cinaracteristic lengths: $\sigma_{0}=\mathrm{e}^{2} \mathrm{kT}$ & A & 10.4 & 8.4 \\
\hline$d=n^{-1 / 3}$ & $\AA$ & 200 & 73 \\
\hline$L=\left(n \sigma_{0}^{2} \ell n \Lambda\right)^{-1}$ & $\AA$ & 170 & 18 \\
\hline Debye $\mathbf{r}_{\mathbf{d}} \equiv \lambda_{\mathrm{d}}$ & $\AA$ & 242 & 61 \\
\hline Arc radius & $\mathrm{mm}$ & 2 & 0.5 \\
\hline
\end{tabular}


b) that a sufficient flux of electrons and ions is supplied by the cathode.

Condition b) means there will be no arc unless the cathode is sufficiently hot. Small (needle type) cathodes may sustain small-current arcs, whereas large, cooled, cathodes require great current to sustain the arc.

The most important difference between the arc studied in the present work and the spark is in the mechanism serving as carrier supply. In sparks, it is a cathodic feedback avalanche, whereas in arcs, one has a simple avalanche supply. In the feedback avalanche, the photun $f^{-} u x$ emanating from the arc creates new charges by photoemission from the cathode. Once the photon flux is sufficiently intense and energetic to overcompensate the loss of carriers, the emitted flux is driven to saturation ty the feedback avalanche. In arcs, on the other hand, one has thermoemission. Atoms and single-charged ions are the predominart species." We confine ourselves to these. 


\section{CHAPTEK 3}

HYDRODYNAMIC EQUATIUNS OF MULIICOMPONENT PLASMAS

(mks)

\subsection{Introduction}

The streaming terms, of which the left side of all kinetic equations are composed, originate with the Liouville equation. The differences among the kinetic equations rest in the collision integral. The Vlasov equation is the simplest kinetic equation of plasmas. Because it is reversible in time, it is not suited to describe an irreversible approach to equilibrium. Consequently, the Vlasov squation is of parlicular use in treating phenorena with very short characteristic times, such as Landau damping, and free plasma oscillations in which collisional damping (responsible for the relixation co equilibrium) plays an insignificant role. Another important kinetic equation is the Fokker-rlanck equation in which the effect of molecular collisions is treated stochastically. Being itself nonlinear, this equation is most often used in a iinear approximation. The divergence at larye interparticle distances in the field of the Coulomb interaction potential is solved by the same cut-off procedure as in the Boltzmann equation. the collision term in the Fokker-Planck equation is calculated on the assumption that there are simultaneous random changes of small vector length in the momentum of the particle, due to its interaction with the other particles of the gas. Consequently, the equation is used for some plasmas in which treatment by a binary collisions formalism may not be justified.

For seven decades after 1872, the Boltzmann equation remained the equation with which to describe irreversible processes in a gas. When one applies the Boltzmann or the Fokker-Planck equation to plasmas, a divergence difficulty arises from the long range of the Coulomb interactions. To avoid it, one inserts a correction for the electric charges' collective effect. We do this by introducing a cut-off equal to the Debye-Hückel length, thus imposing an upper limit on the impact parameter. 
Let us determine whether Boltzmann's binary collision assumption is justified in the present case. We consider the following characteristic. lengths in a plasma with the mean temperature $T$ and mean particle number density $n=d^{-3}$, as in Chap. 6.1 in Wu's book ${ }^{8}(q \cdot v \cdot)$ :

$$
\begin{aligned}
& \sigma_{0}=\frac{e^{2}}{k T} ; \quad d=n^{-1 / 3} ; \quad r_{D}=\left(k T / 4 \pi n e^{2}\right)^{l / 2} \\
& L=\left(n \sigma_{0}^{2} \ln \Lambda\right)^{-1} .
\end{aligned}
$$

These quantities are listed in Table $i$; they confirm the existence of interparticle distances $r$ such that $\sigma_{0}<r<\sigma$, where $\sigma_{0} \ll \sigma \ll d$. This indicates that any two particles "in a Debye sphere" do not "see" other particles between one another, hence their interaction may be considered a binary one for which the molecular chaos assumption is indeed justified. For interparticle distances $r$ such that $\sigma<r<r_{D}$, there are many particles in a sphere of radius $r$ surrounding the particle; but at $r \geqslant r_{D}$, Debye shielding prevails. We have thus snow that it is useful and physically justified for us to proceed with the Boltzmain equation. Methods for solring it were developed by Hilbert (1912), Enskog (1911, 1917), Chapman (1911, 1916), Grad (1949), and others.

\subsection{Selection of a Solution Method}

Already Boltzmann recognized in his papers on viscosity that his equation admits convenient solutions only in the case of Maxwell molecules (Maxwe11, assuming molecules to interact according to his $\mathrm{r}^{-5}$ law, showed that all collision integrals can be solved for $s=5$, with no distribution function being given). Hilbert showed it is possible to obtain the solutiun by solving an infinite series of linear integral equations of the second kind; although this is not a convenient form for the distribution function $f$. Enskog determined $f$ from the Boltzmann equation by a modification of Hilbert's method. He succeeded in 
deriving general formulas for the three coefficients of viscosity, conduction and diffusion in gases of one or several constituents; and calculated the stress tensor up through the third approximation. Chapman, by a different method, arrived at the same results for the same three coefficients. The details of the Enskog-Chapman method are discussed in App. B and in Chapnan and Cowling's book. 9 Important additional reviews are presented in Ferziger and Kaper, 10 Jancel and kahan, 11 and in wu's book. Further contributions on the subject are conseyed in the book of Hirschfelder, Curtiss and Bird. ${ }^{3}$ By the method of Enskug and Chapman, the Boltzmann equation is expanded, in powers of a scaling quantity for the number density, to a set of nonhomogeneous linear integral equations which are solved by way of the hydrodynamic equations for the macruscopic quantities of density, velocity, and temperature. In effect, the initial value problem of the Boltzmann equation ts thus replaced by a series of spatial derivatives of various orders.

In an important paper, 12 crad introduces a different method, which consists in expanding the distribution function in a complete set of orthogonal functions (either Hermite or Sonine polynomials, depending on the coordinate geometry) in the velocity, with coefficients that are functions of the spatial coordinates and time. When one substitutes this expansior in the Boltzmann equation and forms various velocity moments, he obtains for the mo sent equations an infinite set of first order linear differential equations for the coeffiicents $a_{m}(\underline{r}, \underline{t})$. In solving this system, one truncates such as to be left with 13 moments. Because no functional dependence of $f(\underset{\sim}{r}, \underline{v}, t)$ on $n, \underline{v}$, and $T$ is assumed, this method is in principle not restricted, like the Enskog-Chapman method, to small deviations from local equilibrium. However, since the coefficients $a_{\mathbb{m}}(r, t)$ are linear combinations of the inoments of the distribution function, it follows that the set of equations for $a_{m}$ is one of order $\mathrm{m}$, which one would obtain otherwise by operating with the dynamic functions of order in on the Boltzmann equation. Once the expansion for the collision operators $\mathrm{J}_{\mathrm{m}}$ is substituted, one has obtained an infinite set of differential equations for the coefficients 
$a_{m}$, the latter being time derivatives (representing the distribution function). The description of a gas by thirteen variables* (10 in the $2-D$ case, 4 in the 1-D case) is characteristic for Grad's method, as distinguished f:om the Enskog-Chapman method, the latter operating with only the five parameters $n, \underset{\sim}{v}, T$. Grad's method is more effective, therefore, in obtaining good accuracy, though one encounters difficulties in obtaining a solution of the $m$ equations, because the left side contains all the Hermitian coefficients up to, and including, the order $m+1$, whereas the r.h.s. contains the $m$ coefficients in the expansion of $J_{m}$.

A detailed account of the Enskog-Chapman method is presented in App. B, where the hydrodynamic equations of the Lorent? gas are derived, and its transport coefficients calculated. The form of the streaming operator in the multicomponent plasma with magnetic field is developed in the section immediately following.

Let us agree that characters with the diacritical denote tensors, their order** being determined by the physical nature. Velocities, for example, arefirst order tensors (i.e., vectors) denoted $\stackrel{\mathrm{v}}{\mathrm{V}}$.

\section{3 The Multicomponent Plasma}

\subsubsection{Multicomponent Averages}

Let $f_{i}(\underset{\sim}{r}, \underset{v}{v}, t)$ be the distribution function of the $i-t h$ component, $v_{i}$ the actual velocity of an $i-t h$ component particle, a bar denote the average value of a dynamic function, and $d \underset{\sim}{ }$ a differential

\footnotetext{
* One counts 20 equations for the first through third moment, but he reduces the set to 13 by dropping the linearly nearly dependent ones; hence the name "13-moment method".
}

${ }^{* *}$ Order is sometimes called valence, or rank, there being no convention. 
volume in velocity space $d \underset{\sim}{v}=d v_{x} d v_{y} d v_{z}$. One may thus define for the i-th component the following quantities.
a) Number density................. $n_{i}=\int E_{i} d v_{i}$;
b) Average velocity ............... $\bar{z}_{i}=\frac{1}{n_{i}} \int z_{i} f_{i} d v_{i}$;
c) Mass uverage velocity.......... $v_{0}=\frac{{ }_{1}}{p}{ }_{0}{ }^{2} n_{i} m_{i} \bar{l}_{i}$
d) Overall density of the gas at a given point.............
D) Peculiar velocity of Chapman $\ldots . . v_{i}=\underline{v}_{\underline{i}}-\underline{v}_{0}$ and cowling where one has........ $i_{i} \rho_{i} V_{i}=0$
f) Diftusion velocity w.r.t.
the mass average velocity of
the gas................ $\bar{v}_{\underline{i}}=\bar{v}_{\dot{i}}-v_{0}=\frac{1}{n} ;\left(v_{i}-v_{0}\right) f d v$;

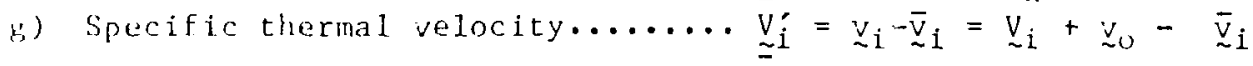 reduced to $\bar{v}_{i}$ where one has....... $\bar{v}_{i}=0$

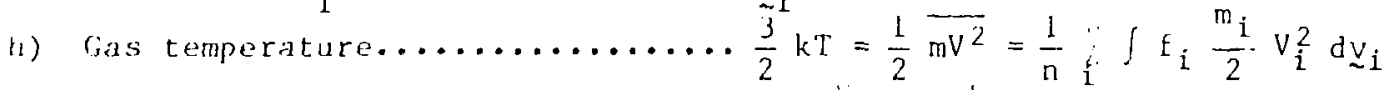
i) Total pressure tensor..........

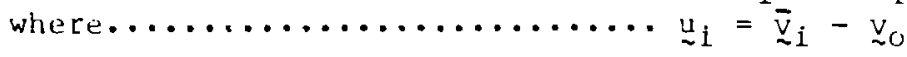
j) Partial pressure tensor $\ldots \ldots \ldots . e_{i}^{\prime}=n_{i} m_{i} v_{i}^{i} v_{i}^{\prime}$
k) Thermal flux vector $\ldots \ldots \ldots \ldots \ldots q q_{i}^{\prime}=\frac{1}{2} \rho_{i} \frac{v_{i}^{2}}{v_{i}}$
The quantities defined $i: j$ ) and $k$ ) are calculated in App. B.

The definitions a) - f) are the customary ones, given in Chap. 7.2 a of Hirschfelder, Curtiss and Bird's book. 'is The quantities a) - k) are discussed also in Chap. 6.2 of Jancel and Kahan's book. 11

3.3.2. Derivation of the Maxwel 1 Equation for a Huticumponent plasma in the Presence of an E.r. Field

Consider encounters with impact parameter b between particles of the species $i$ and $j$. The probable number of collisions in $d b d t$ between particles having $\underline{v}_{i}$ in $d \underline{\sim}_{i}$ and those having ${\underset{\sim}{j}}_{j}$ in $d \underline{\sim}_{j}$, $b$ db being a scattering cross section, is given by the collision integral. The collisions change $\underset{\sim}{ }$ and hence decrease or increase $f$ to $f^{\prime}$. We follow the formalism developed in Jancel and Kahan's book ${ }^{1}$ (Chaps. 5 
and 6) and write the Boltzmann equation for the i-th component in terms of the streaming operator $/ t$ and the collision integral:

$$
\frac{\not f_{i}}{\not t}=i_{j} \iint\left(f_{i} f_{j}-f_{i} f_{j}\right) g_{i j} b d b d v_{i} \equiv i_{j}\left(\frac{\delta f_{i}}{\delta t}\right)_{j} \cdot
$$

Maxwell's transport equation is a macroscopic equation, obtained by multiplying Eq. (l) by the dynamic function $\psi_{i}(r, \underset{v}{v}, t)$ and integrating over velocity space:

$$
\int \Phi_{i} \frac{\partial f_{i}}{\delta t} d v_{i}=\int d_{i} \frac{\delta f_{i}}{\delta t} d v_{i}
$$

where $\delta \mathrm{f}_{\mathbf{i}} / \delta \mathrm{t} \equiv !\left(\delta \mathrm{f}_{\mathbf{i}} / \delta \mathrm{t}\right)_{\mathbf{j}}$ is a sum over all the species. We have from Liouville's equation, discussed in App. C (q・v•):

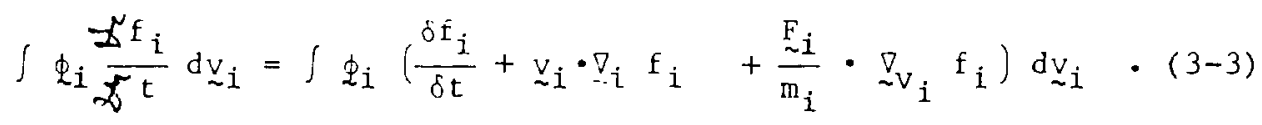

With the chain rule, we easily obtain the following identities:

$\int \Phi_{i} \frac{\partial f_{i}}{\partial t} d v_{i}=\frac{\partial}{\partial t} n_{i} \overline{\Phi_{i}}-n_{i} \overline{\frac{\partial}{\partial t} \Phi_{i}}$

$\int \Phi_{i} v_{i x} \frac{\partial f_{i}}{\partial x} d v_{i}=\frac{\partial}{\partial x} n_{i} \overline{\Phi_{i} v_{i x}}-n_{i} v_{i x} \frac{\partial \Phi_{i}}{\partial x}$

$\frac{F_{i}}{m_{i}} \int \Phi_{i} \frac{\partial f_{i}}{\partial v_{i x}} d \underline{v}_{i}=\iint\left[\Phi_{i} f_{i}\right] \sum_{v_{i x}=-\infty}^{c} d v_{i y} d v_{i z}-\int f_{i} \frac{\partial f_{i}}{\partial v_{i x}} d \underline{v}_{i}=-n_{i} \frac{\overline{\partial \Phi_{i}}}{\partial v_{i x}} \cdot(3-3 c)$ 
By collecting, the three identitias, we can write Maxwell's transfort equation in the form

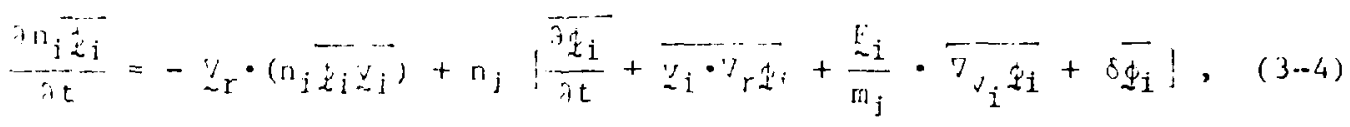

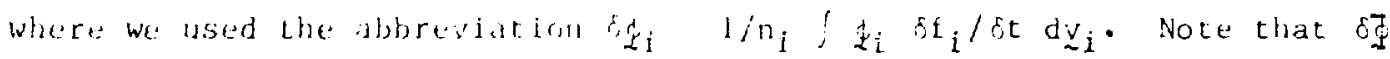
is just the albreviation defined, not os be confused with the abbreviation: $\lambda_{t}$ ilite $\lambda_{x}$ diax uscd elsewhere in the treatise.

In the end, we will necd lie herat flow equation, therefore the variablos should be changed from the present $\underline{v}_{i}$ to a new $\underline{V}_{i}$ dependency, where we recali $\underline{\sim}_{i}(\underline{r}, t)=\underline{v}_{i}(t)-\ddot{\sim}_{0}(r, t)$. It follows that the time derivatives in $\mathrm{Eq}$. (4) transform like

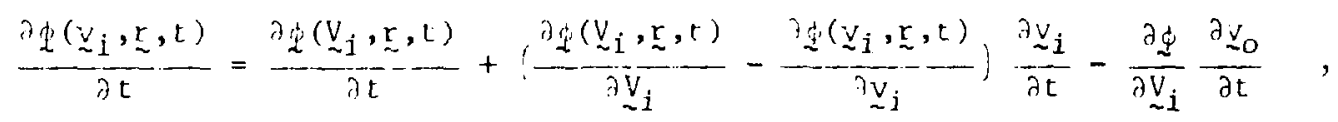

where $\partial{\underset{\sim}{v}}_{j} / \partial t=0$ is needed so the middle term on the r.h.s. may vanish and not complement to the total derivatives on both sides of the equation, which is consistent with the text statement immediate y following Eq. (8). Thus we replace

$$
\begin{aligned}
& \frac{\partial \Phi\left(\underline{\sim}_{i}, \underset{\sim}{r}, t\right)}{\partial t}+\frac{\partial \Phi_{i}\left(\underline{\sim}_{i}, \underset{\sim}{r}, t\right)}{\partial t}-\left[\nabla_{\underset{\sim}{V}} \Phi_{i}\left({\underset{\sim}{\mathrm{V}}}_{i}, \underset{\sim}{r}, t\right)\right] \cdot \frac{\partial{\underset{\sim}{o}}_{0}}{\partial t} \\
& \nabla_{r} \Phi_{i}\left({\underset{\sim}{i}}_{i}, r, t\right)+\nabla_{r} \Phi_{i}\left({\underset{\sim}{i}}_{i}, \underset{\sim}{r}, t\right)-\left[\nabla_{v_{i}} \Phi_{i}\left({\underset{\sim}{i}}_{i}, \underset{\sim}{r}, t\right)\right] \cdot \nabla_{r \sim_{0}} \cdot
\end{aligned}
$$

With these substitutions, Eq. (4) becomes 


$$
\begin{aligned}
& \frac{\partial}{\partial t} n_{i} \overline{\phi_{i}}=-\nabla_{r} \cdot\left\{n_{i}\left[\Phi_{i}\left(\underline{v}_{0}+v_{i}\right)\right]\right\}+n_{i}\left\{\frac{\partial \Phi_{i}}{\partial t}-\left(\nabla_{v_{i}} \Phi_{i}\right) \cdot \frac{\partial \underline{v}_{0}}{\partial t}+\underline{v}_{0} \cdot \overline{\nabla_{r} \Phi_{i}}+\right. \\
& \left.+\overline{v_{i} \cdot \nabla_{r} \Phi_{i}}-\overline{\left(\nabla_{v_{i}} \Phi_{i}\right.}\right) \cdot\left(\underline{v}_{0} \cdot \nabla_{r}\right) \underline{v}_{0}-\overline{\nabla_{v_{i}} \Phi_{i} \cdot\left(v_{i} \cdot \nabla_{r}\right)} \underline{v}_{0}+ \\
& +\frac{F_{i}}{m_{i}} \cdot \overline{v_{v_{i}}} \Phi_{i}+\delta \overline{\Phi_{i}} !
\end{aligned}
$$

Let us introduce a special notation for the ubstantial derivative or derivative following the motion, $D / D t: \partial / \partial t+\underline{v}_{0} \cdot v_{r}$, by which any ambiguity with the total derivative $d / d t$ (i.e., one w.r.t. all the independent variables, $\underline{r}, \underline{v}, t)$ will be avoided.

We recal1 ${ }^{*}$ the tensor identity $\underline{\sim}_{\mathrm{V}} \Phi \cdot\left(\underset{\sim}{V} \cdot \nabla_{\mathrm{r}}\right) \underset{\sim}{\mathrm{V}} \equiv\left(\underline{\sim}_{\mathrm{V}} \Phi L \mathrm{~V}:{\underset{\sim}{\mathrm{r}}}_{\sim}^{\mathrm{V}}\right.$ so we may write Eq. (5) in the following form

$$
\begin{aligned}
& *\left(\begin{array}{c}
\frac{\partial \phi}{\partial v_{x}} \\
\cdot \frac{\partial \phi}{\partial v_{y}}
\end{array}\right)\left(\begin{array}{c}
v_{x} \frac{\partial v_{x}}{\partial x}+v_{y} \frac{\partial v_{x}}{\partial y} \\
v_{x} \frac{\partial v_{y}}{\partial x}+v_{y} \frac{\partial v_{y}}{\partial y}
\end{array}\right)=\frac{\partial \phi}{\partial v_{x}}\left(v_{x} \frac{\partial v_{x}}{\partial x}+\frac{\partial \phi}{\partial v_{y}} v_{y} \frac{\partial v_{x}}{\partial y}\right)+\frac{\partial \phi}{\partial v_{y}}\left(v_{x} \frac{\partial v_{y}}{\partial x}+v_{y} \frac{\partial v_{y}}{\partial y}\right) \\
& \equiv v_{x}\left(\frac{\partial \phi}{\partial v_{x}} \frac{\partial v_{x}}{\partial x}+\frac{\partial \phi}{\partial v_{y}} \frac{\partial v_{y}}{\partial x}\right)+v_{y}\left(\frac{\partial \phi}{\partial v_{x}} \frac{\partial v_{x}}{\partial y}+\frac{\partial \phi}{\partial v_{y}} \frac{\partial v_{y}}{\partial y}\right) \equiv \\
& \equiv T_{r}\left\{\left(\begin{array}{ll}
\frac{\partial \phi}{\partial v_{x}} v_{x} & \frac{\partial \phi}{\partial v_{y}} v_{y} \\
\frac{\partial \phi}{\partial v_{y}} v_{x} & \frac{\partial \phi}{\partial v_{y}} v_{y}
\end{array}\right)+\left(\begin{array}{cc}
\frac{\partial v_{x}}{\partial x} & \frac{\partial v_{y}}{\partial x} \\
\frac{\partial v_{x}}{\partial y} & \frac{\partial v_{y}}{\partial y}
\end{array}\right\}\right.
\end{aligned}
$$




$$
\begin{aligned}
& \frac{D\left(n_{i} \Phi_{i}\right)}{D t}+n_{i} \phi_{i}{ }^{7} \cdot V_{0}+\nabla_{r} \cdot\left(n_{i} \overline{\phi_{i} V_{i}}-n_{i} \overline{\frac{J_{\phi_{i}}}{D t}}+\overline{V_{i} \cdot \nabla_{r} \Phi_{i}}\right. \\
& \left.+\left(\frac{F_{i}}{m_{i}}-\frac{D v_{0}}{D t}\right) \cdot \overline{7_{V_{i}} t_{i}}-\overline{\left(\nabla_{V_{i}}\right) V_{i}}: \nabla_{r} v_{0}\right\}=n_{i} \delta \bar{\phi}_{i} .
\end{aligned}
$$

'hius far, we have been using for the external torce function the $v$ locity independent form ${\underset{\sim}{F}}_{i}(\underset{r}{r} \underline{\sim})$. This is because, originally, ${\underset{\sim}{i}}_{i}$ was defined by the Liouville equation, where $\mathrm{F}_{\mathrm{i}}=\mathrm{m} \mathrm{d}_{\mathrm{i}} / \mathrm{dt}$. In the Lorentz force $Z$ e $(\underset{\sim}{E}+\underset{\sim}{v} \times \underset{\sim}{B})$, however, the electrodynamic term $\underset{\sim}{v} \times \underset{\sim}{B}$ is a velonity

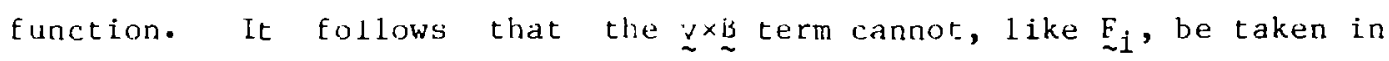
rront of the integral in the Maxwell transport equation. Let us proceed with the integration. Consider the streaming terms in the Boltzmann $(\vec{B})$ and Maxwe $11(\boldsymbol{M})$ equations, i.e.,

$$
\begin{aligned}
& \tilde{B} \equiv \frac{\widetilde{\gamma f_{i}}}{\partial t}=\frac{\partial f_{i}}{\partial t}+v_{i} \cdot \nabla_{n} f_{i}+\frac{F_{i}}{r_{i}} \cdot \nabla_{v_{i}} f_{i} ;
\end{aligned}
$$

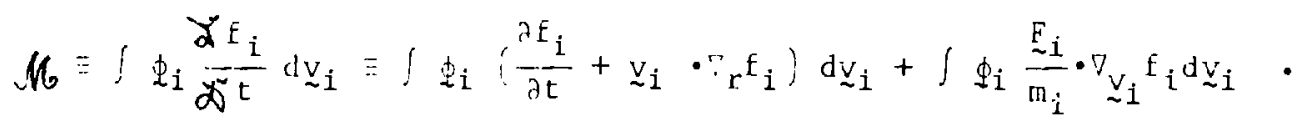

Substitute for $\underset{\sim}{F_{i}} / m_{i}$ the electrodynamic force $\psi_{i}$ e/m $\underset{\sim}{A} \equiv Z_{i}$ e/m $\cdot(\underset{\sim}{z} \times \underset{\sim}{b})$. The source term of $\mathcal{M}$ may thus be written

$$
\int \phi_{i} \sum_{x} A_{x} \frac{\partial f_{i}}{\partial v_{i x}} d{\underset{\sim}{i}}_{i},
$$

where the summation over the ccordinate related three algebraic terms of $\stackrel{A}{\sim} \underset{\sim}{\underset{\sim}{i}} \mathrm{~F}_{\mathbf{i}}$ is denoted. For any of these three terms, we gei 


$$
\int \Phi_{i} A_{x} \frac{\partial f_{i}}{\partial v_{i x}} d v_{i}=\iint\left[\Phi_{i} A_{x} f_{i}\right]_{\underline{v}_{i x}=-\infty}^{\infty} d v_{i y} d v_{i z}-\int f_{i} \frac{\partial A_{x} \phi_{i}}{\partial v_{i x}} d v_{i}
$$

where the first term on the r.h.s. vanishes at the boundaries $\left(f_{i}=0\right.$ for $v \rightarrow \infty)$. Hence,

$$
\int \Phi_{i} A_{x} \frac{\partial f_{i}}{\partial v_{i x}} d \underline{v}_{i}=-n_{i} A_{x} \frac{\partial \Phi_{i}}{\partial v_{i x}}-n_{i} \Phi_{i} \overline{\frac{\partial A_{x}}{\partial v_{i x}}} ;
$$

where, in turn, the last term is zero identically, by definition [i.e., $A_{x}=A_{x}\left(v_{y}, v_{z}, B_{y}, B_{z}\right)$ is independent of $\left.v_{x}\right]$. We are thus left with

$$
\frac{z_{i} \mathrm{e}}{m_{i}} \int \Phi_{i}(\underset{\sim}{v i} \times \underset{\sim}{B}) \cdot \nabla_{v_{i}} f_{i} d v_{\sim}=-n_{i} \frac{z_{i} e}{m_{i}}\left(\underset{\sim}{v_{i}} \times \underset{\sim}{B}\right) \cdot \nabla_{v_{i}} \Phi_{i}
$$

Hence, if the Lorentz force is the only external force, the Maxwell equation in the $\bar{v}_{i}$-dependent form of Eq. (4) becomes

$$
\begin{aligned}
& \frac{\partial n_{i} \overline{\Phi_{i}}}{\partial t}=-\nabla_{r} \cdot\left(n_{i} \phi_{i} \underline{\sim}_{i}\right) \\
& \quad+n_{i}\left[\frac{\partial \Phi_{i}}{\partial t}+\overline{v_{i} \cdot \nabla_{r} \Phi_{i}}+\frac{z_{i} e \underset{\sim}{e}}{m_{i}} \cdot \overline{\nabla_{v_{i}} \Phi_{i}}+\frac{z_{i} e}{m_{i}} \overline{\left(\underline{v}_{i} \times B\right) \cdot \nabla_{v_{i}} \Phi_{i}}+\delta \bar{\delta}\right] \cdot(3-7)
\end{aligned}
$$

Similarly, for $V_{i}$ as an independent variable, where we replace in Eq. (6) the term ${\underset{\sim}{i}}_{i} / m_{i} \cdot \overbrace{V_{i}} \Phi_{i}$ by the new expression 


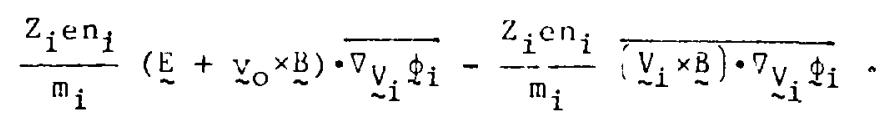

With this substitution, Eq ( 6 ) becones:

$$
\begin{aligned}
& \frac{D n_{i} \overline{Q_{i}}}{D t}+n_{i} \Phi_{i} \nabla_{r} \cdot \underline{v}_{0}=-\nabla_{r} \cdot\left(n_{i} \overline{\Phi_{i} v_{i}}\right)+n_{i} \frac{J_{\Phi_{i}}}{\overline{D t}}+\overline{V_{i}} \cdot \overline{\nabla_{r} \Phi_{i}}+ \\
& +\left[\frac{Z_{i} e}{m_{i}}\left(\underset{\sim}{E}+{\underset{\sim}{0}}_{0} \times \underline{\sim}\right)-\frac{D v_{0}}{D t}\right] \cdot \overline{\nabla_{v_{i}} x_{i}}+
\end{aligned}
$$

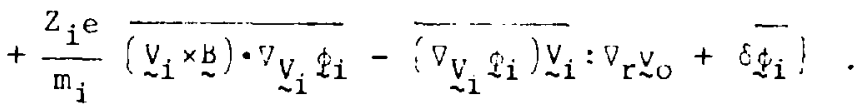

We can now substitute the privileged quantitits, formally denoted $\$$, to obtain the moment equations. Because $\phi_{i}$ represents a set of velocity functions, the terms in $\partial \phi_{i} / \partial t$ vanish in Eqs. (7) and (8), the average peculiar velocity being zero and the rate of change in the average velocity being small, cf. the footnote in the section immediately following.

\subsubsection{The Moment Equations}

$\underline{\phi_{i}}=\mathrm{m}_{i}$ leads to the continuity equation. Because $\nabla_{v_{i}} \Phi=0$ in this case, the Lorentz force is inconsequential and the continuity equation for the $i-t h$ constituent reads

$$
\frac{\partial n_{i}}{\partial t}+\nabla_{r}\left(n_{i} \bar{v}_{i}\right)=\int d \underline{v}\left(\frac{\partial f}{\partial t}\right)_{c} .
$$

The integral on the right is the rate of ionization of the positive and 
negative chargis. The rate is negative for the neutral atorns. Because $\mathrm{F}_{\mathrm{i}} \mathrm{m}_{\mathrm{i}}=\mathrm{E}_{\mathrm{i}}^{\prime} \mathrm{m}_{i}^{\prime}$, in elastic collisions no rollision integral appears.

$\Phi_{i}=m_{i \sim i} \underline{i}_{i}$ sy substitutirg $\Phi_{i}$ in each term in $E_{q} \cdot(7)$, the following expressions appear:

$$
\begin{aligned}
& \frac{\partial}{\partial t} n_{i} \Phi_{i}=n_{i} m_{i} \frac{\partial V_{i}}{\partial t}-\nabla_{r} \cdot\left(n_{i} \Pi_{i} \bar{V}_{i}\right) ; \quad(c f \cdot E q \cdot 9) \\
& \nabla_{r} \cdot\left(n_{i} \overline{\Phi_{i} \bar{v}_{i}}\right)=\nabla_{r} \cdot\left(n_{i} m_{i} \overline{\left(\underline{v}_{i}+V_{i}^{\prime}\right)\left(\underline{v}_{i}+V_{i}^{\prime}\right)}\right)=\nabla_{r} \cdot\left(n_{i} m_{i} \bar{v}_{i} \bar{v}_{i}\right)+\nabla_{r} \cdot\left(n_{i} m_{i} \overline{V_{i} V_{i}}\right) ; \\
& \nabla_{r} \cdot\left(n_{i} m_{i} \underline{v}_{i} \underline{\sim}_{i}\right)=n_{i} m_{i}\left(\underline{v}_{i} \cdot \nabla_{r}\right) \bar{v}_{i}+n_{i} m_{i} \bar{v}_{i}\left(\nabla_{r} \cdot \underline{\sim}_{i}\right)+\underline{v}_{i}\left(\bar{v}_{i} \cdot \nabla_{r} n_{i} m_{i}\right) ; \\
& v_{r} \cdot\left(n_{i} m_{i} \overline{V_{i} V_{i}}\right)={ }^{\prime} \cdot R_{i}^{\prime}
\end{aligned}
$$

By collecting terms, we obtain from Eq. (7)

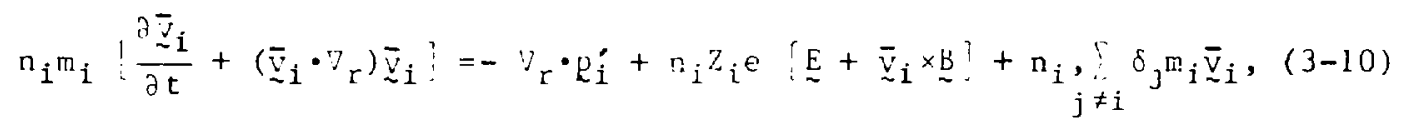

where the collision integral

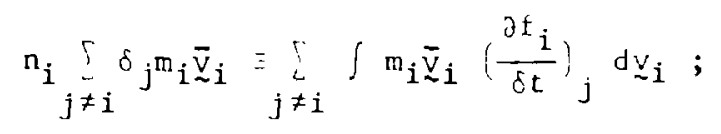

and where

$$
\sum_{i} \sum_{j \neq i} n_{i} \delta_{j} m_{i} \bar{v}_{1}=0
$$

For instance, in a plasma composed of the components $j \equiv$ (e) (i.e., 
electrons), and $i$ : (i) (i.e., ions), we have $n_{e} \hat{j}_{i}\left(m_{e} \bar{v}_{e}\right)+n_{i}$ $f_{e}\left(m_{i} \bar{v}_{i}\right)=0$ in the stationary state.

$$
\underline{\Phi_{i}}=\mathrm{m}_{i} / 2 \mathrm{v}_{i} \cdot 2 \text {. We have } \overline{\overline{7}_{V_{i}} \Phi_{i}}=\overline{m_{i} V_{i}^{\prime}}=0 \text { and obtain from }
$$

Eq. (7) the heat balance equation, where the following term needs to be evaliated.

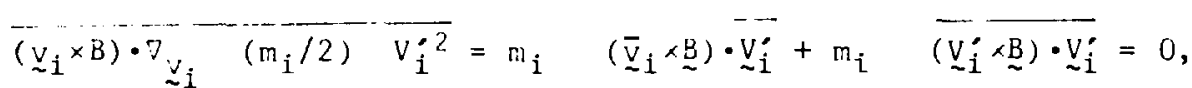

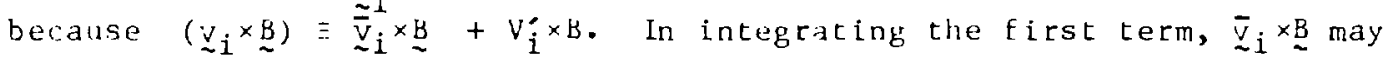
be treated as a constant, whereas the second term must be integrated in its entirety. The first term vanishes, because $\bar{\sim}_{i}$ does, and the second term is zero by vector identity. We may thus write the heat balance equation:

$\left.\frac{1}{2} \frac{\partial\left(\rho_{i} \overline{v_{i}^{2}}\right)}{\partial t}+\nabla_{r} \cdot \frac{1}{2} \rho_{i} \overline{v_{i}^{2} \bar{v}_{i}}\right)+\nabla_{r} \cdot q_{i}^{j}+p_{i}^{i}: \nabla_{r \sim i} \bar{v}_{i}=\sum_{j \neq i} n_{i} \delta_{j} \frac{n_{i}}{2} \overline{v_{i}^{\prime 2}} \cdot(3-13)$

In the first two terms, we have $\left.\left(\rho_{i} / 2\right) \overline{v_{i}^{2}}=3 / 2\right) k I_{i}$. The heat flux

$$
\underline{q}_{i}^{\prime}=\left(\rho_{i} / 2\right) \overline{v_{i}^{2}} \underline{v i}_{i}^{\prime}=-1 \gamma_{r} T_{i},
$$

as shown in App. B. The last term on the left is easily recognized as $R_{i}^{\prime}: V_{r} \bar{v}_{i}=p V_{r} \cdot \bar{v}_{i}$, because $p_{i}^{\prime}=p^{\prime}$, where ${ }_{\sim}$ is the unit tensor, $p$ being the hydrostatic pressure. ${ }^{*}$ For the collision term, the sime argurent applies as for the equation of motion. We nay thus write

*It is shown in App. B (Eqs. 23 and 33) that the quantities on the 1.h.s. of _our transport Eqs. (9), (10), and (13) represent zeroth order

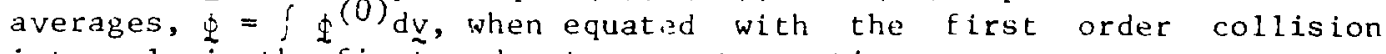
integrals in the first order transport equations. 


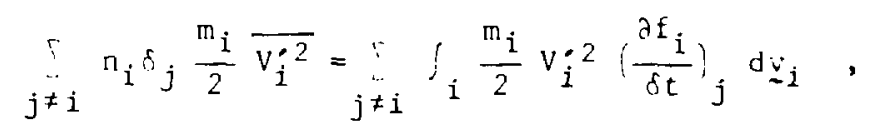

where

$$
\underset{i}{\therefore \neq i} \Omega_{i} \& \frac{m_{i}}{2} \overline{v_{i}^{2}}=0 .
$$

If one develops the heat balance equation from $\phi_{i}=m_{i} V_{i}^{\prime} v_{i}^{\prime}$ as the dynamic function, then one obtains a moment equation with more than twelve terms, many of which are functions of the Lorentz force. Most of the terms cancel or they are tensor identities of zero. The remaining tensor equation reduces to Eq. (13) identically. 
CHAPLEK 4

SAHA'S TRLLATINE FUR A THREL COMPUNENT NOLISOTHEKLAL. PLASMA

(mks units)

Saha's equation results fror extremizing the free energy with respect to the number density varied in an ionization process which changes

$$
n_{i}, n_{e}, n_{0} t 0 n_{i}+5 n_{i}, n_{e}+5 n_{e}, n_{0}-\delta_{0} .
$$

Consider a sufficiently dilute gast consisting of the thrte components: electrons, ions, and neutral gas. Each component is an ideal system is the Boltzmann approximation, in the absence of external fields, with chemical reactiuns among the thres compontents, i*e.,

$$
\begin{aligned}
& (\ddot{z}+1)+u=\% \\
& y_{\ell+1}=1 ; v_{u}=1 ; v_{\ell}=-1 \text {, }
\end{aligned}
$$

where the $v_{i}$ ar" the coefficients of the reaction (stoechiometric coefficients). We need the free energy for the component $r$ of the reaction, i.e.,

$$
A_{Y}(T, v, n)=-n_{\gamma} k T_{Y}\left[\ln \frac{v}{n_{Y} h^{3}}\left(2 \pi m_{\gamma} k T_{\gamma}\right)^{3 / 2} z_{i \gamma}+1\right] \text {, }
$$

which derives from the partition function

\footnotetext{
${ }^{*}$ We assume canonical distribution, hence not cryogenic temperature and not very high density.
} 


$$
Z_{Y}=Z_{\text {IrY }} Z_{I Y}
$$

where ${ }^{2} \operatorname{Tr}$ is the translational partition function

$$
{ }^{2} \mathrm{Tr}=\mathrm{A}^{-3}
$$

related to the de Brörlie wavelength $\Lambda$, and the incerna! fartition tunction

$$
z_{I Y}=\ddot{i}_{i} s_{i Y} e^{-\varepsilon} i \gamma / k I_{Y}
$$

is the sum over the $i$ internal energy Ievels, $\varepsilon_{i}$, deterinined by quantum mechanics with biy being the statistical weintint of the degeneracy of the $i^{\text {th }}$ level. The law of mass action is a statement of conservation of energy and requires tinat the Helmholcz free energy $A(t, v, n)$ be an invariant of the ceaction. We calcilate, therefore,

$$
\frac{\partial A}{\partial n}=\frac{\partial A}{\partial n_{Y}} \frac{\partial n_{Y}}{\partial n}
$$

which is obtained from Eqs. (2) and (5) for the constituent $\gamma$, i.e.,

$$
\frac{\partial A}{\partial n_{Y}}=-\ell n\left[\frac{v}{n_{\gamma} h^{3}}\left(2 \pi m_{\gamma} T_{\gamma}\right)^{3 / 2}{\underset{i}{i} g_{i \gamma}}^{-\varepsilon_{i \gamma} / k T}\right\} ? .
$$

For the sum over the corstituents, in thermodynamic equilibrium, we obtain 


$$
\frac{\partial A}{\partial n}=-\ln \prod_{\gamma}\left[f\left(n_{\gamma}, m_{Y}, T_{\gamma}\right)\right]^{\partial n_{\gamma} / \partial:}
$$

where the expunents can be expressed by the reaction coefficients, i.e., $\delta n_{\gamma}=v_{\gamma} \delta n$. We set Eq. (7) equal to zero and obtain

$$
l=\pi ! \frac{v}{n_{\gamma} h^{3}}\left(2 \pi m_{\gamma} k T_{\gamma}\right)^{3 / 2} \zeta g_{i j} e^{-\varepsilon_{i \gamma} / k T} ! v_{\gamma} .
$$

To bring Eq. (8) in the conrentional form of Guldberg and Waage, I4 we take the pressure $p_{Y}=k n_{Y} T_{Y} / V$ to the 1.h.s. and get

$$
\left.\prod_{Y} p_{\gamma}^{U_{Y} T_{Y}}=\prod_{\gamma} \frac{k T_{Y}}{h^{3}}\left(2 \pi m_{\gamma} k T_{Y}\right)^{3 / 2} \sum_{i}^{r} g_{i \gamma} e^{-E_{i \gamma} / k T}\right]^{{ }_{\gamma} T_{Y}}
$$

where each component has been raised to the power $T_{\gamma}$. If we now extend the sim and product indices $i$ and $y$ only over electrons with $\mathrm{p}_{\mathrm{e}}, \mathrm{m}_{\mathrm{e}}, \mathrm{T}_{\mathrm{e}}$, $v_{e}$; neutral gas with $\mathrm{p}_{z}, \mathrm{~m}_{z}, T_{z}, v_{z}$; and ions with $\mathrm{P}_{z+1} ; \mathrm{m}_{z+1}=\mathrm{m}_{z}=\mathrm{m}_{\mathrm{h}}$, $\mathrm{T}_{z+1}=\mathrm{T}_{z}=\mathrm{T}_{\mathrm{h}}$, $\mathrm{T}^{*}$ for the excited state, and $\nu_{z+1}$, we get after raising the entire equation to the power $-\mathrm{T}_{\mathrm{e}}^{-1}$,

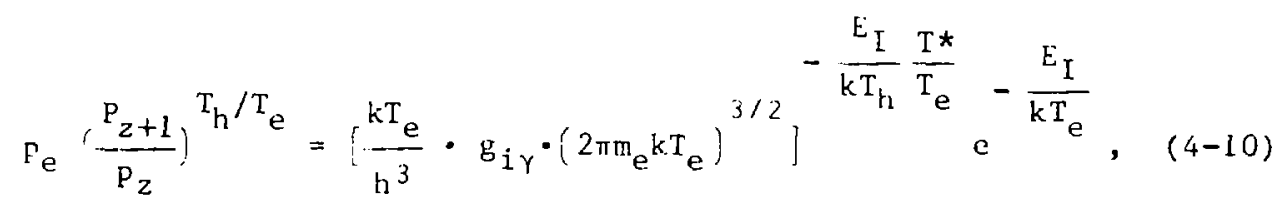

where $E_{I}=E_{z+1}-E_{z}$ is the ionization potential. To write $E_{i}$. (1) in the form of the Saha equation, we introduce the degree of ionization 


$$
x=\frac{n_{Z+1}}{n_{Z}+i} \cdot
$$

In addition to the variation of the free internal energy of the corstituent components of the reaction, one must consider the corresponding external variation due to Coulomb interactions, because changes in the Debve potentials are being induced by the raction. It has been shown ${ }^{15}$ that this effect in terms of an equivalent, hypothetical, change in ionization potential, amounts to $E_{I}-\Delta E_{I}$ where $\Delta E_{I}=(z+1) e^{2} / r_{d}(e s u)$. For the ionization of neutrai atoms, $z=0$, and $\Delta E_{i}$ may presently be neglected, because one obtains $\Delta E_{I} / E_{1}=10^{-6}$ with the numbers from Table 1 .

Observe that. the system considered consists of one sub-system for each constituent. Each subsystem is an ensemble of particles with a canonical distribution, where tempezature, volume, and number of particles in the system are the independent variables. The system is submerged in a reservoir whose thermodynamic variables are supposed to be known. For this reason, the reservoir provides the flow balance of particles.

It has been postulated ${ }^{16}$ that the total gaskinetic pressure is invariant. We tried to impese a different invariant of the reaction by assigning invariance to the partial pressure of the neutral particles: We believed that only the neutrals' pressure was maintained by the gas reservoir surrounding the arc. We found this assumption would lead to the nonphysical condition, where in the limit of vanishing spatial gradients in the energy flow balance, an identity in $\partial \mathrm{T}_{0} / \partial t$ results. Hence, we take the total pressure to be the invariant of the reaction. By writing Eq. (10) for the three-component nonisothermal plasma, and not allowing for excited states, we get 


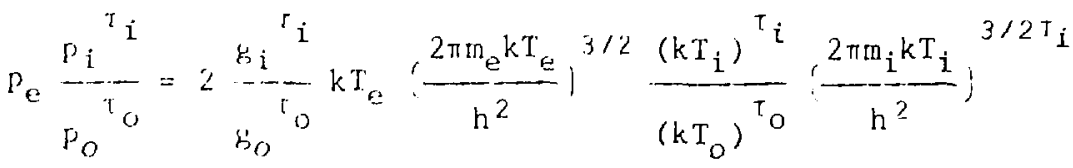

$$
\begin{aligned}
& \frac{2 \pi m_{0} k T_{0}}{h^{2}} j^{-3 / 2 \tau_{0}} e^{-E_{1} / k T_{e}},
\end{aligned}
$$

where we use the abbreviations $\mathrm{T}_{i}=\mathrm{T}_{i} / \mathrm{T}_{e} ; \mathrm{I}_{0}=\mathrm{T}_{0} \mid \mathrm{T}_{e}$. The invariance condition, in themodynamic equilibrium, is given by

$$
p_{0}^{0}=p_{0}+p_{0}+p_{i}=n_{0} k T_{0}+n_{e} k T_{e}+n_{i} k I_{i}=\frac{n_{0}^{0}}{r_{i}}\left|(I-x) k T_{0}+x\left(k T_{e}+k T_{i}\right)\right|
$$

where $r$ is the parameter fixed by the invariance condition and where the superscript o denotes an ininital value. We get

$$
\begin{gathered}
\left.n=\frac{T_{0}^{0}}{T_{0}^{0}}: 1-x+x \frac{T_{e}+T_{i}}{T_{0}}\right), \\
\frac{n_{0}}{n_{0}^{0}}=\frac{T_{0}^{0}}{T_{0}} \cdot \frac{1-x}{1-x+x \frac{T_{e}+T_{i}}{T_{0}}},
\end{gathered}
$$

and 


$$
\frac{n_{e}}{n_{0}^{0}}=\frac{n_{i}}{n_{i}^{0}} \frac{x}{1-x+x \frac{T_{e}+T_{i}}{T_{0}}},
$$

where we confined ourselves to considering neutral and singly charged particles.

The invariance condution is substituted in Eq. (12) by the relationship

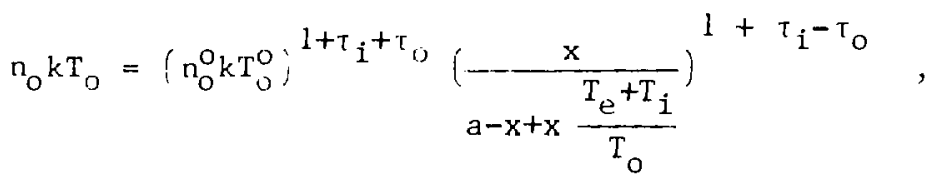

where the Eirst expression in parentheses is identified with $\mathrm{p}_{\mathrm{O}}^{\mathrm{o}}$, which is the invariant total pressure. With this substitution, one can write

$$
\begin{gathered}
\left(\frac{n}{n_{0}}\right)^{\tau_{0}}\left(\frac{p_{0}^{0}}{1+\tau_{i}+\tau_{0}} \frac{n_{0}}{n}\right)^{1+\tau_{i}-\tau_{0}}=g\left(\frac{2 \pi}{h^{2}}\right)^{3 / 2\left(l+\tau_{i}-\tau_{0}\right)}\left(\frac{m_{0} m_{0}}{m_{0}}\right)^{3 / 2} e^{-E_{I} / k T_{e}} \\
\frac{\left(k T_{i}\right)^{3 / 2 \tau_{i}}}{\left(k T_{0}\right)^{3 / 2 \tau_{0}}}\left(k T_{e}\right)^{5 / 2+\tau_{i}-\tau_{0}} .
\end{gathered}
$$

Henceforth, we use the ionization ratio $x=n / n_{0}$ instead of the ionization degree defined earlier. The invariance condition requires a flow such that 


$$
\mathrm{n}_{0}=\frac{p_{0}^{O}}{x\left(k T_{e}+k T_{i}\right)+\overline{k T_{0}}}
$$

Equation (18) is Saha's equation extended to the three-component nonisothermal plasma. It establishes the thermodynamic equilibrium relationship among number densteles of three species (electrons, single-charged ions, and neutrals), dependent of the temperatures. Because we use Saha"s equation later as one of the relationships to close the system of hydrodynamic equations, we need the differential dependency among the variables of the Saha equation. After differentiating Eq. (18) and setting $T_{e}=T_{i}, n_{e}=n_{i}$, we can write the relationship in the following convenient form

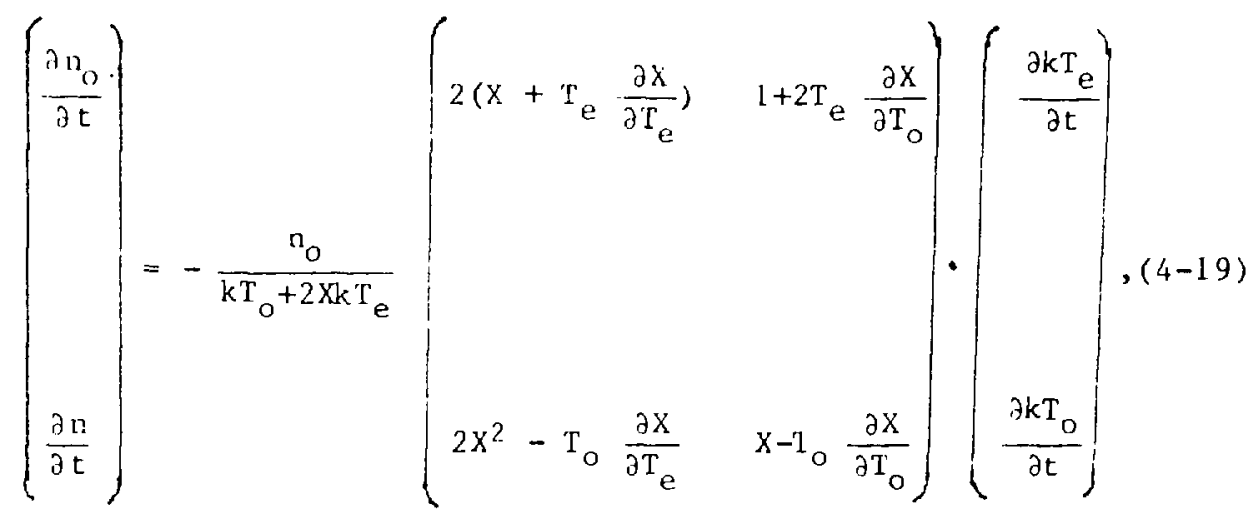

where the derivatives of $X$ are obtained from the following two relationships derived directly frum $E q$. (18)

$$
\left(1+\frac{2-\tau_{o}}{2 X+\tau_{o}}\right) \frac{k T_{o}}{X}\left(\frac{\partial X}{\partial k T_{e}}\right)=5-2 \tau_{0} \frac{X+1}{2 X+\tau_{0}}+\frac{E_{I}}{k T_{e}}+\tau_{o} \text { znk, }
$$




$$
\left(1+\frac{2-\tau_{0}}{2 X+\tau_{0}}\right) \frac{k T_{0}}{X}\left(\frac{\partial X}{\partial k T_{0}}\right)=\frac{-3}{2}+\frac{2-\tau_{0}}{2 X+\tau_{0}}-\ell n K,
$$

where

$$
k=\left(\frac{2 \pi m_{0} k T_{o}}{h^{2}}\right)^{3 / 2} \frac{k T_{e}}{p_{o}^{o}}\left(2 x+t_{o}\right) .
$$

Finally, we determine the total statistical weight $g$ for the quantum mechanical degeneracies of the atomic nitrogen system. From Herzberg, 17 we find $g_{0}=4, g_{i}=3$ (ortho), and one has of course $g_{e}=2$, yie]ding

$$
g=\frac{g_{e} g_{i}^{T_{i}}}{g_{0}^{T_{0}}}
$$

The ground state ionization potential is given as $15.6 \mathrm{eV}$. In our numerical work, we used $g=2$ and $E_{I}=16 \mathrm{eV}$. 
CHAPTEK 5

F.ASSTL COLLLSIUNS

(esu, exceptions noted)

We confine ourseives to those renarks on Rutherford scattering that may complement Chap. 3, and to the topic of encounters between electrong and neutral particles. As regards the latter, we refer to the work by ingelhardt et $a 1 .,{ }^{1 /}$ where the effective momentum transfer collision frequency is triven by

$$
v_{(0)} / n_{0}=2 \cdot 10^{-9} \mathrm{cos}^{3} / \mathrm{s} \text {, at }>1.4 \mathrm{ev} \text {. }
$$

A synopsis of Spitzer's treatise 25 on Rutherford scattering in the following, form may be useful. Let the local equilibrium distribution in velocity of the test particles--iee., the ones being, scattered by the field particles ${ }^{*}$--be given by the one-particle reduced distribution function

$$
f^{(o)}(v)=-\frac{n}{\pi^{3 / 2}} \frac{1}{\bar{v}^{3}} c^{-(v / \bar{v})^{2}}
$$

where $n$ is the local number density per unit volume, and $\bar{v}$ the most probable test particle speed. We assume, by $\mathrm{Eg}$. (1), spatial homogeneity. Interaction with the field particles is introduced by way of a new phase space in which the spatial independence of the distribution function, Eq. (1), is augmented to a new dependence, which is one of the impact parameter. In other words, the impact parameter

\footnotetext{
* "Field particles" have been naned in analogy with the astrophysics term "field star"; they are stacionary, whereas the test particles are to test effects of their own motion.
} 
assumes the place of the sparial variables; the field particles themselves are not represented by their distribution function. We simply have constructed a phase space in the variables $(b, v)$. We can thus define a spectal Debye cell in $(b, r)$ space, $1 . e \cdot$, a cylindrical shell with length $v$ and cross section $2 \pi b \mathrm{db}$. The number of field particles found in the shell is thus $2 \pi b$ db. The number of field particles found in the shel1 is thus $2 \pi b v n_{i} d b$, where $n_{i}$ is the number density per unit phase space volume, of the field particles.* The integral over the cylinder diverges for $b \rightarrow \infty$. Spitzer sets an upper and a lower limit corresponding to the Debye radius and the short range interaction radius, thus defining the parameter $\Lambda$.

Time is still a complementary independent variable, needed to describe the evolution of the interaciton. Hence, one may calculate the characteristic times of the evolution. Spitzer obtains, cf. Eq. (8-25) in Rose and Clark,

$$
\tau_{e i}=\frac{\left(2 m_{e}\right)^{1 / 2} W_{e}}{4 \pi e^{4} n_{i}} \frac{1}{\ell n \Lambda} \text {, }
$$

where $w_{e}$ is the total kinetic energy (corresponding to the total velocity square average). Because the drift speed is small, compared with the random speed, Spitzer equates

$$
\mathrm{w}_{\mathrm{e}} \simeq \frac{3}{2} \mathrm{k}_{\mathrm{e}}
$$

Hence, the collision frequency is defined by

\footnotetext{
*our derivation of this classical cross section offers a useful alternative to the one ordinarily given.
} 


$$
v_{e i}=1.379 \cdot 10^{-12} \frac{n_{i} Z^{2} \ln \Lambda}{\left(\frac{k T_{e}}{\epsilon}\right)^{3 / 2}}(M K S)
$$

whe re

$$
\Lambda=1.539 \cdot 10^{13} \frac{\left(\frac{\mathrm{kT}_{\mathrm{e}}}{\mathrm{e}}\right)^{3 / 2}}{\mathrm{n}_{\mathrm{e}}^{1 / 2}} \text { (MKS) }
$$

We emphasized our identification of the independent variables in Spitze:"s interaction phase space because the same end result may be obtained by the following alternative argument inviting a possible misconception.

The momentum change of the test particles has been expressed in a relationshipl9 that includes not only the spatial length of an interaction volume in phase space, but also the impact parameter, both being independent variables. Hence, one excessive independency has entered into the argument. The spatial length is then used to define a collision cross section. The correct relaxation time, i.e., Eq. (2), is obtained by correlating the two over the random kinetic energy. A relationship is in use 20 for the resistivity, which relates the correct cross section erroneously by the drift velocity, to the collision Erequency, the latter being wrong. Refer to App. B-5 for a more rigorous treatise. 


\section{CHAPTEK 6}

CYLINDRICAL ARC ENVELOPE FUNCTIUN

(mks units)

We will see that the Lagrangian of the charge labeled $i$ in an external EM field is given by

$$
L=-\frac{1}{2} m_{i} v_{i}^{2}-q_{i} \phi+q_{i} \vec{A} \cdot v_{i}
$$

where $\phi$ and $\vec{A}$ are the external electric and magnetic potentials, respectively, evaluated at the position of the particle with mass $m_{i}$, velocity $v_{i}$, and charge $q_{i}$. We have thus written the Lagrangian for one of the charges of the system--under a given motion of the other charges. From this, one can easily find the Lagrangian of the whole system. By taking the sum over the system, one obtains

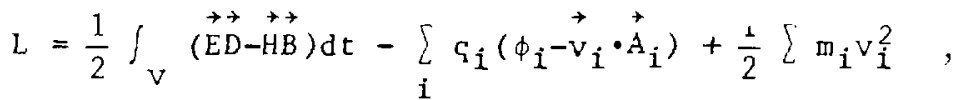

where

$$
\vec{E}=-\nabla \phi=\frac{\partial}{\partial t} \vec{A}
$$

and

$$
\vec{B}=7 \times \vec{A},
$$

where $B$ and $D$ are the flux densities, and $H$ and $E$ the field gradients (sometimes called intensities) in the magnetic and electric field, 
respectively. The three terms on the r.h.s. of Eq. (1) are the Lagrangians, from left to right, of the field, the field-particle interaction, and the particles.

To operate with Euler's derivative $(\partial / \partial q-d / d t \partial / \partial \dot{q})$ on the lagrangian, three choices of generalized coordirates $(q, \dot{q})$ are possible, i.e.,

$$
\begin{aligned}
& \phi, \dot{\phi} \\
& \vec{A}, \dot{A} \\
& x, \dot{x}=v .
\end{aligned}
$$

Two of Maxwell's equations, i.e.,

$$
\begin{aligned}
& \nabla \cdot \vec{D}=\rho \quad(\text { from }, \dot{\phi}), \\
& \nabla \times \vec{H}-\frac{\partial}{\partial t} \vec{D}=\vec{J} \quad(\text { from } \vec{A}, \dot{A}),
\end{aligned}
$$

are thus obtained; the other two having been used to define the potentials in Fqs. (2) and (3). Let us turn to the kinetic coordinates $x_{\gamma}, \dot{x}_{Y} \equiv v_{Y}$ to obtain the desired kinetic equations. Cylindrical coordinates are suitable for the general geometry of the arc chamber and contacts. We assume azimuthal symmetry and thus obtain two expressions for each of which d'Alembert's principle obtains. Hence they vanish independently, i.e.,

$$
\frac{\partial L}{\partial z}-\frac{d}{d t} \frac{\partial L}{\partial v_{z}}-Q=0
$$




$$
\frac{\partial L}{\partial r}-\frac{d}{d t} \frac{\partial L}{\partial v_{r}}-Q=0
$$

The terms $Q$ are required by d'Alembert's principle for nonconservative systems with frictional forces present. We set $Q=0$, perhaps in analogy with zero on the r.h.s. of the Boltzmann equation leading to the conservation equations of fluid dynamics.

We assume that the current $i$ is impressed so the current density be position-independent within the arc column, i.e.,

$$
j \equiv j_{z}=i / \pi r^{2}
$$

and the electrostatic gradient

$$
\frac{d}{d z} \phi=j_{z} / \sigma=i / \pi \sigma r^{2}
$$

We calculate the radius and length of the cylindrical column shown cross-hatched in Fig. Id and neglect the disk-like (shaded) ends. Because the magnetic field at radii exceeding the electrodes' inner radius $R$ is an invariant of the arc column's linear dimensions, that field is not being considered. We set

$$
\begin{aligned}
& \phi=\frac{d \phi}{d z} \ell=i \ell / \pi \sigma r^{2}, \\
& B_{\theta}=B(\rho=\bar{r})=\frac{\mu_{i}}{2 \pi \bar{r}}\left(\frac{\bar{r}}{r}\right)^{2}=-\frac{\partial A}{\partial \bar{r}},
\end{aligned}
$$

from which we get 


$$
A(\rho=\bar{r})=\frac{\mu_{i}}{4 \pi} \mathbf{i}\left(\frac{\bar{r}}{r}\right)^{2} .
$$

By substituting this in Eq. (1), we get the Lagrangian in cylindrical coordinates, i.e.,

$$
L=\frac{i \mu}{4 \pi}\left(\frac{\varepsilon}{\mu} \frac{4}{\sigma^{2}} i \ell\left(\frac{R^{2}}{r^{4}}\right)-\frac{i \ell}{4}-i \ell \ell n \frac{R}{r}+\frac{4 \ell}{n^{2} j \mu}-\frac{1}{r^{2}} \varphi_{i}-\vdots q_{i} \vec{v}_{i} \frac{r_{i}^{2}}{r^{2}} ! .\right.
$$

To be consistent with the assumption of a position independent current density within the arc, we require $r_{i} / r \equiv n=$ constant. We thus get the two conjugate derivatives

$$
\begin{aligned}
& \frac{\partial L}{\partial r}=\frac{i \mu}{4 \pi}\left[i \ell\left(-\frac{16 \varepsilon R^{2}}{\mu \sigma^{2} r^{5}}+\frac{1}{r}\right)-\frac{8 \ell}{\mu^{2} \sigma \mu r^{3}} \vdots q_{i}\right] \\
& \frac{\partial L}{\partial \ell}=\frac{i \mu}{4 \pi}\left[i\left(\frac{\varepsilon}{\mu} \frac{4}{\sigma^{2}} \frac{R^{2}}{r^{4}}-\frac{1}{4}-\ln \frac{R}{r}\right)+\frac{\vdots q_{i}}{n^{2} \sigma \mu r^{2}} !\right.
\end{aligned}
$$

$(6-14)$

Furthermore,

$$
\frac{\partial L}{\partial \vec{v}_{i}}=\frac{i \mu}{4 \pi} n^{2} G_{i}
$$

hence

46 


$$
\int \frac{d}{d t} \frac{\partial L}{\partial \vec{v}_{i}}=\frac{\mu n^{2}}{4 \pi} \frac{d}{d t}\left(i \sum q_{i}\right) .
$$

The vector notation on the 1.h.s. of Eq. (15) is to indicate that derivatives with respect to the velocity components $v_{r}$ and $v_{z}$ are meant, there being two equations in all. Because the signs of the products $q_{i} v_{r i}$ and $q_{i} v_{z i}$ are intrinsically positive (positive charge going right or negative charge going left), we may substitute in Eq. (15) $q_{i}=e\left(n_{e}+n_{i}\right)$ to obtain

$$
-\frac{d}{d t} \frac{\partial L}{\partial v_{z}}=-\frac{d}{d t} \frac{\partial L}{\partial v_{r}}=-\frac{\mu n^{2} e}{4 \pi} \frac{d}{d t} i\left(n_{e}+n_{i}\right) .
$$

By summing Eqs. (13), (16), and (14), (16), respectively, we obtain the zero variations w.r.t. $(r, \dot{r})$ and $(\ell, i)$ :

$$
\begin{aligned}
& 0=i \ell\left(\frac{1}{r}-\frac{16 \varepsilon R^{2}}{\mu \sigma^{2} r^{5}}\right)-\frac{8 \ell e\left(n_{e}+n_{i}\right)}{n^{2} \sigma \mu r^{2}}-\frac{n^{2} e}{i} \frac{d}{d t}\left(n_{e}+n_{i}\right) i, \quad(0-17) \\
& 0=i\left(\frac{\varepsilon}{\mu} \frac{4}{\sigma^{2}} \frac{R^{2}}{r^{4}}-\frac{1}{4}-\ell n \frac{R}{r}\right)+\frac{e\left(n_{e}+n_{i}\right.}{n^{2} \sigma \mu r^{2}}-\frac{\eta^{2} e}{i} \frac{d}{d t}\left(n_{e}+n_{i}\right) i,(6-18)
\end{aligned}
$$

which are two equations in the four variables $r, \ell, i$, and $\left(n_{e}+n_{i}\right)$. Equation (18) is an ODE containing only $r, i$, and $\left(n_{e}+n_{i}\right)$ as variables. It may be rewritten for $i=I=$ const in a more convenient form, i.e.,

$$
\alpha\left(n_{e}+n_{i}\right)-\beta \cdot \frac{d}{d t}\left(n_{e}+n_{i}\right)=-\gamma .
$$


Euler's rquation returns the function defining the arc envelope for which the laigonfiar. is statinnary. Because we use cylindrical coordinates, the minimun is represented by $r=$ const, 1 = const. The Euler-Lagrange formalism requires a constant current density profile in Eq. (8) to yield the nbserved right cylindrical arc envelope. 
CHAPTER ?

CURRENT EQUATLAN AND SUMMARY UF EQUATLUNS

(raks units)

Let us rewrite the hydrodynamic kqs. $(3-9),(3-10)$, and (3-13) in a corm suitable for numerical evaluation in cyli.idrical coordinates. It is easily verified the equations are identical in cys and mks units, the factor of transformation from one to the other being 1 or $\sqrt{4 \pi \varepsilon_{0}}$ depending on the equation and not the individual tern. As regards conservation of momintum, it may be written either as a momentum cr current equation. We use the latter. Let $a$ denote the unit positive charge; the electron and ion currents are then given by ${ }^{\star} j_{e}=e n \mathbb{v}_{e}$, $\dot{j}_{i}=e n \bar{v}_{i}$, with the total current

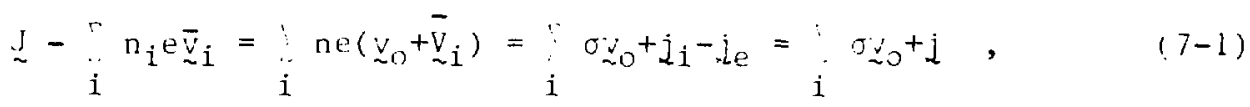

where o $\mathrm{V}_{0}$ is the electronic convection current due to a collective motion of the ionized gas, and $i$ is the conductica current of the electrons and ions relative motion. There being no chance of confusion, we use the subscrif ${ }^{2} i$ as a general label for the specier, as well as for ions specifically when $e$ is used for the electrons, and o

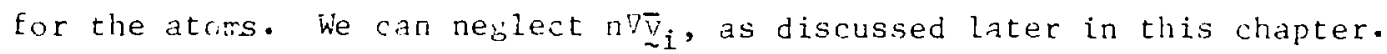
The mass conservation $\ddot{\mathrm{Eq}} \cdot(3-9)$ becones therefore

$$
\frac{\partial n_{i}}{\partial t}+\vec{v}_{i} \cdot \nabla n_{i}: \frac{\delta n_{i}}{\delta t}
$$

\footnotetext{
${ }^{*} \mathrm{~V}_{\epsilon}$ and $\mathrm{v}_{\mathrm{i}}$ are defined to point in opposite directions; hence, because e is defined as a positive charge, $j=e n\left(y_{j}-y_{e}\right)$.
} 
where the r.h.s. is to account for the gains and losses from the inelastic collisions discussed in Chap. 4.

\subsection{Current Equation}

Let us proceed to the equation of currents obtained from the momentum conservation $\mathrm{Eq} \cdot(3-10)$. We move the pressure gradient term to the l.h.s. and proceed as follows:
a) Simplify the $1 . h . s_{.}$, reading $n_{i} n_{i}\left[\partial \bar{v}_{i} / \partial t+\left(\bar{v}_{i} \cdot \nabla\right) \bar{v}_{i}\right]+7 \cdot q_{i}^{\prime}$;
b) Simplify the set of all streaming terms;
c) Write the collision terms;
d) Combine the streaming and collision terms to the new form.

We recall the definition of Chapman and Cowling's peculiar velocity $\underline{v}=\underline{v}-\underline{v}_{0}, i \cdot e \cdot$,

$$
{\underset{v}{v}}_{i}={\underset{\sim}{v}}_{i}+\underline{v}_{0}={\underset{\sim}{V}}_{i}+\bar{v}_{i}
$$

and proceed with step (a). It is easy to show that

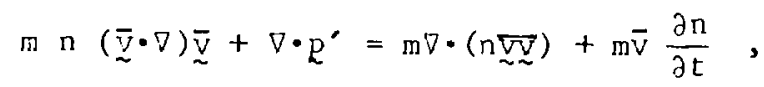

hence, Eq. (10) in Chap. 3 becomes

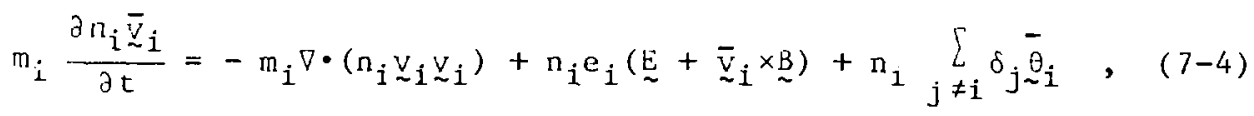

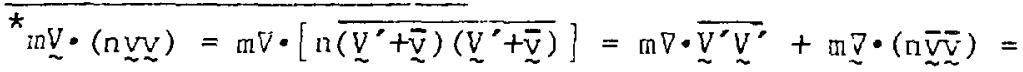

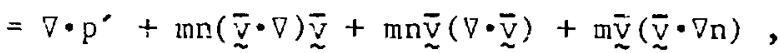

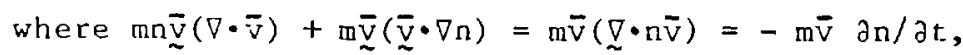
and where we substituted the continuity Eq. (2).
} 
where we abbreviated $m \bar{v} \equiv \overline{9}$. After multipiying Eq. (4) by $e_{i}$, one can show that the first term on the r.h.s. in F.q. (4) becomes

$$
e \nabla \cdot(n \underset{\sim}{v})=\nabla \cdot \frac{e}{u} p+\nabla \cdot\left(\underline{\sim}_{0}\right)+\nabla \cdot\left(\underline{v}_{0} J\right)
$$

where the last two terms may be neglected, because $v_{0}$ and $j$ are small, compared witk the peculiar velocity and its e-multiple; more so since the terms are quadratic by the products of $v_{0}$, $j$. The electrokinetic pressure tensor may be replaced by the scalar pressure $p_{i}$, cf., the text near the end of App. $\mathrm{B-4}$. It is justified to neglect $\mathrm{F}_{i} / \mathrm{m}_{i}$, which is small when compared with $\mathrm{p}_{\mathrm{e}} / \mathrm{m}_{\mathrm{e}}$. The set of all streaming terms may thus be written

$$
\frac{\partial j}{\partial t}=-\nabla \frac{c_{i}}{m_{i}} p_{i}+\frac{e_{i}^{2}}{m_{i}} n_{i} \underset{\sim}{E}+\frac{e_{i}^{2}}{m_{i}} n_{i} \vee_{0} \times \underset{\sim}{B}+\frac{e_{i}}{m_{i}} \underset{i}{j} \underset{\sim}{B} .
$$

The signs follow from $\operatorname{sgn}\left(e_{i} E\right)$, Eq - (5) being derived from the momentum equation multiplied by $e_{i}$. If $E q \cdot(5)$ is explicitly written for electrons and ions, then we obtain, by subtracting and adding that pair of equations, these two new relationships:

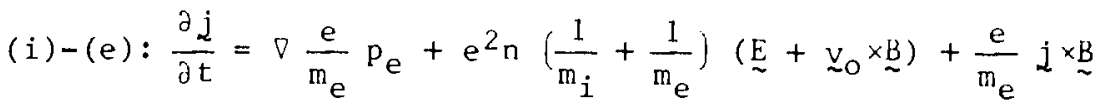

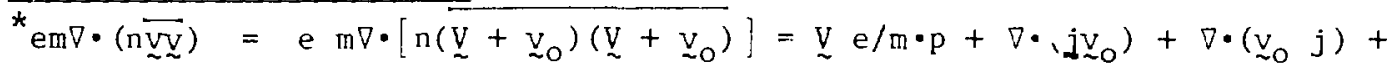
$\nabla \cdot\left(\sigma \underset{\sim}{\bigvee_{0}} \underset{0}{ }\right)$, where the first term on the r.h.s. is the electrokinetic pressure tensor. 


$$
\text { (i) }+(e): \quad m_{i} \frac{\partial \dot{\alpha}_{i}}{\partial t}+m_{e} \frac{\partial j_{e}}{\partial t}=0=-\gamma e p_{e}+e j_{e} \times \underset{\sim}{i} .
$$

The collision terms are still neglected in Eqs. (6) and (7), thus keeping only the streaming terms. The l.h.s. in Eq, (7) is zero identically, because $m_{e} n_{e} \bar{v}_{0}+m_{i} n_{i} \bar{v}_{i}=0$, and of $\bar{v}_{e}$ and of $\bar{v}_{i}$ being defined to point in opposite rirections.

Let us proceed tu step (c) in our outline and write the collision terms. * We have

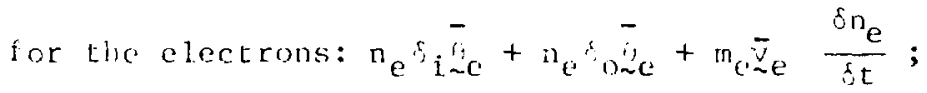

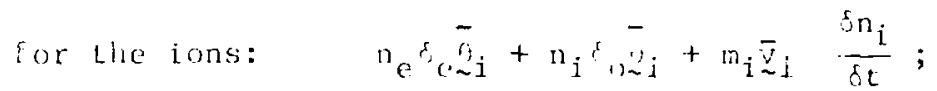

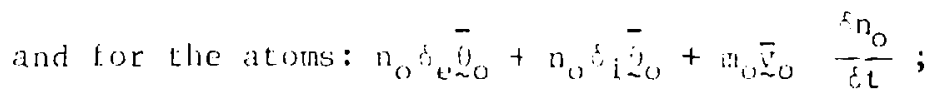

where $n_{e}=n_{i}=n$, and $x=n /\left(n+n_{0}\right)$. The first wo terms in each of the expressions (8)-(10) represent elastic interactions, the third terins the contributions from int lastic encounters. Because the sum of the elastic collision terins vanishes when taken over all three expressions $(8)-(10)$, tire rate-of-change operator $\delta_{k}$ must have the property

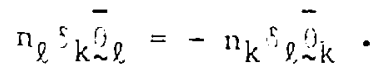

The operator is defined by

*Jancel and Kahan's book, $l$ ll Eqs. $(6-99)-(6-104)$, p. 302-303. 


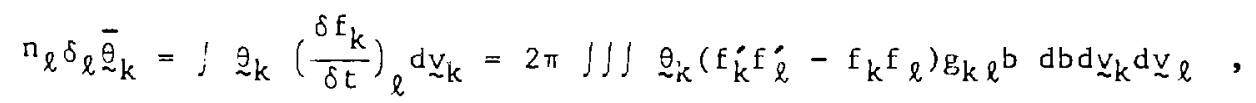

from which the antisymmetry just discussed follows, $g_{k l}=-g_{1 k}$ being the relative velocity. Hence the explicit form for our three component system:

$n_{e} \delta_{i \sim e} \bar{\theta}_{e}=-n_{i} \delta_{e} \overline{\underline{\theta}}_{i}=-n_{e} m_{e} \nu_{e i}\left(\underline{\sim}_{e}-\bar{v}_{i}\right)=-n_{e} m_{e} \nu_{e i}\left(\bar{v}_{e}-\bar{v}_{i}\right)=\nu_{e i} \frac{m_{e}}{e} j_{j}$

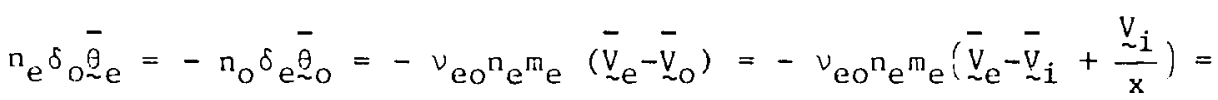

$$
\nu_{\text {eo }} \frac{m_{e}}{e} j-v_{e o} \frac{m_{e}}{e} \frac{j}{x}
$$

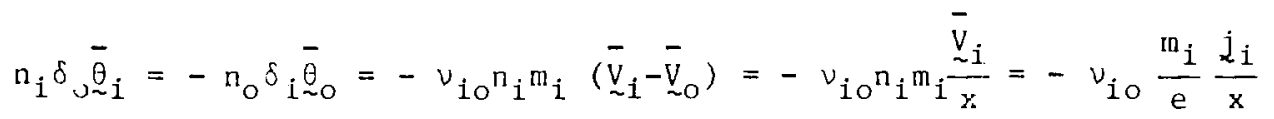

Following the same procedure of adding and subtracting from one another the streaming expressions for the ions and electrons, we derive new expressions for the collision terms. For electrons ninus ions, we get

$$
\text { en } \delta_{e} \stackrel{\bar{\theta}}{i}_{i}-e n \delta_{i \sim e} \bar{\theta}_{\sim}+e n \delta_{0 \sim i} \bar{\theta}_{i}-e n \delta_{o \sim e} \bar{\theta}_{e}+\left(e m_{e} \bar{v}_{e}-e m_{i \sim i} \bar{v}_{i}\right) \frac{\delta n}{\delta t},
$$

or, explicitly, by neglecting $m_{e} / m_{1}$ against 1 :

$$
-\left(2 v_{e i}+v_{e o}+\frac{v_{i o}}{x}+\frac{2}{n} \frac{\delta n}{\delta t}\right) m_{e j}
$$


Similarly, by adding electrons and ions, i.e.,

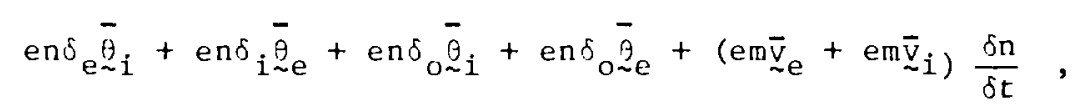

and neglecting $m_{e} / m_{i}$ against 1 , we finally have

$$
+v_{\text {eo }} m_{\mathrm{j}} \mathbf{j}
$$

We can thus proceed with the step (d) in our outline, combining the Eqs. (7) and (15):

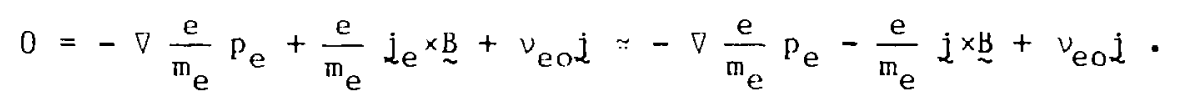

Similarly, with Eqs. (6) and (14), where we substitute Eq. (16) to eliminate the hydrostatic pressure gradient. We omit the inelastic collision term, because the inelastic events are dominated by thermal ionization, in our case. Hence,

$$
\frac{\partial \mathfrak{j}}{\partial t}=\frac{e^{2} n}{m_{e}}\left(\underset{\sim}{E}+\underline{\sim}_{0} \times \underset{\sim}{B}\right)-\left(2 v_{e i}+\frac{v_{i o}}{x}\right) m_{e} \mathfrak{j} .
$$

Under the stationary condition $\partial \mathbf{j} / \partial t=0$, we have Ohm's law in $\mathrm{Eq} \cdot(17)$. By having substituted Eq. (16), we put in Eq. (17) all the information from momentum conservation of electron and ion encounters. Convection current may be neglected in Eq. (17). This applies in an arc with cylindrical or slightly conical envelope, if we assume rotational symmetry (i.e., $\partial / \partial \phi=0$ ) in addition to the radial component being small, $v_{o r} \simeq 0$. From classical E.M. theory, we have 
$\nabla \cdot$ env $_{\sim_{0}}=0=$ en $\nabla \cdot v_{0}$, causing $\frac{\partial}{\partial z} \bigvee_{0 z}$ to vanish when $\frac{\partial}{\partial \phi} \underline{v}_{0} \phi$ does, as postulated, and $v_{\text {or }} \propto r$ as in a conical arc envelope.

\subsection{Energy Flow Equation}

Let us proceed to the energy flow equation. In the text following Eq. (3-13) (1.e., the energy flow equation) and near the end of App. B-4, we discussed the reduction of the double dot term. We showed that $\mathrm{R}: \nabla \underset{\sim}{\overline{\mathrm{q}}}=\mathrm{R}^{\nabla} \underset{\sim}{\overline{\mathrm{v}}}=\mathrm{nkT} \underset{\sim}{\mathrm{I}} \nabla \cdot \underset{\sim}{\bar{v}}$. At the same occasions we discussed the heat flow vector $g$, cf. the text following Eq. (62) in App. B. In conclusion, we can write, omitting the species subscripts,

$$
\nabla \cdot \mathrm{g}=-\nabla \frac{\lambda}{\mathrm{m}} \nabla \mathrm{kT}=-\frac{\lambda}{\mathrm{k}} \nabla^{2} \mathrm{kT}=-\underset{\sim}{\mathrm{E}} \cdot \underset{\sim}{\mathrm{J}}+\mathrm{P}_{\mathrm{rad}}+\mathrm{P}_{\text {turb }},
$$

where the heat source $\lambda / \mathrm{k} \nabla^{2} \mathrm{kT}$ has intuitively been decomposed into a term E.J for the ohmic heating, one for the radiation loss, and one for the heat loss by turbulent flow. The only term deserving further discussion is $\nabla \cdot(k T n \vec{v})$. For charged species, it follows from $\nabla \cdot J=0$ that $\nabla \cdot(\mathrm{kT} \mathrm{n} \underset{\sim}{\bar{v}})=\underset{\sim}{J} / \mathrm{e} \cdot \nabla \mathrm{kT}$. To sum up, the heat flow equation may be written in the form

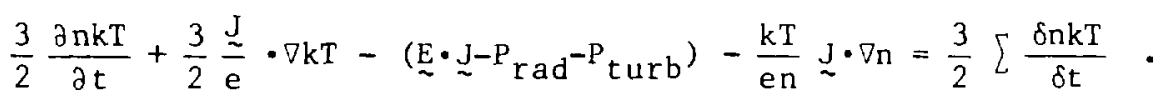

The collision term in Eq. (18) includes the contributions from elastic collistons and ionization, the sum over all species being zero. Hence,

$$
\begin{gathered}
\frac{\partial k T_{i}}{\partial t}=\frac{1}{n}[-\frac{J_{i}}{e} \cdot \nabla k T+\frac{2}{3}\left(\underset{-}{E} \cdot J_{i}-P_{i r a d}-P_{i} \text { turb }\right)+\frac{2}{3} \frac{k T_{i}}{e n} \underbrace{}_{i} \cdot \nabla n_{i}- \\
\left.\left(\frac{2}{3} E_{I}+k T_{i}\right) \frac{\delta n_{i}}{\delta t}-\sum_{j \neq i} \nu_{i j}\left(k T_{i}-k T_{j}\right)\right],
\end{gathered}
$$


where $E_{T}$ is the ionization energy, and $\delta n / \delta t$ the source term in the continuity equation. The terms J/e are positive for ions, negative for electrons, consistent with the equation's derivation. We assume $\nabla \mathrm{kT}$ to be small.

\section{3 Summary of Equations}

Writing Eqs. (17) and (19) in matrix form for the individual species, we get

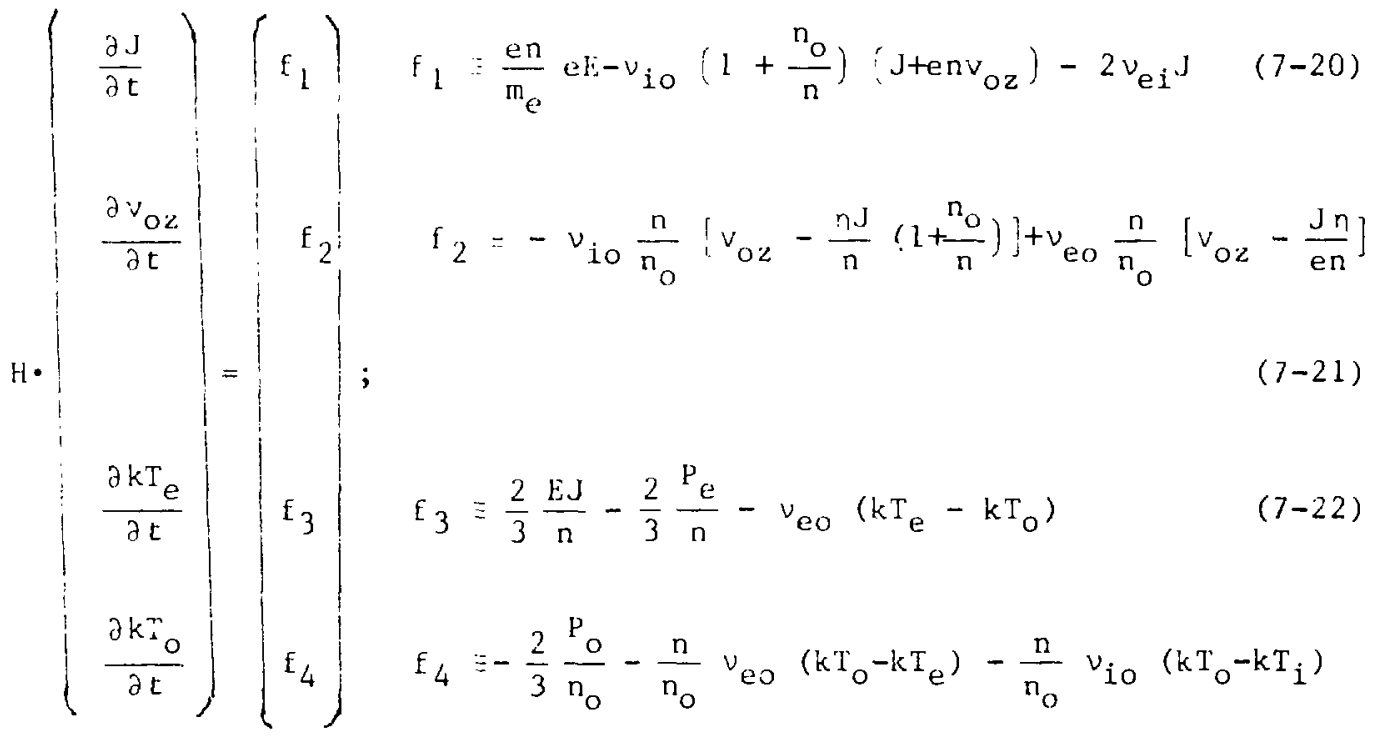

where $\eta \equiv \mathrm{m}_{\mathrm{e}} / \mathrm{m}_{i}$ and where all time derivatives have been moved to the l.h.s., yielding the matrix 


$$
H=\left\{\begin{array}{ccc}
1 & 0 & 0 \\
0 & 1 & a_{11} \frac{v_{O z}}{n_{O_{2}}} \\
0 & 0 & 1+a_{21} \frac{k T_{e}+\frac{2}{3} E_{i}}{n} \\
0 & 0 & a_{11} \frac{k T_{0}}{n_{0}}
\end{array}\right.
$$

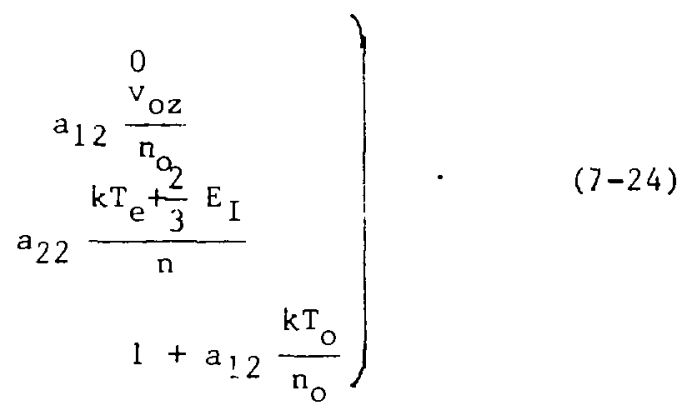

The matrix elements of $a_{i j}$ are defined in $E q \cdot(4-19)$ where, for example

$$
a_{12} \equiv-n_{0} \frac{1+2 T_{e} \frac{\partial x}{\partial T_{0}}}{T_{0}+2 X T_{e}}
$$

and where the derivatives of $X$ are given explicitly by Eqs. (4-20) and $(4-21)$. We have defined $J=-J_{e}+J_{i}$. We assume $T_{e}=T_{i}$ as before.

To gain an order of magnitude estimate of the collision frequencies, let us use the values from Table $I$, from which the following numbers derive.

$\begin{array}{llll}\mathrm{T} & \mathrm{kK} & 16 & 20 \\ \mathrm{n} & \mathrm{m}^{-3} & 1.3 \cdot 10^{23} & 2.6 \cdot 10^{24} \\ \mathrm{n}_{\mathrm{O}} & \mathrm{m}^{-3} & 1.8 \cdot 10^{23} & 4.0 \cdot 10^{24} \\ \ell \mathrm{n} \Lambda & & 4.2 & 3.0 \\ v_{\mathrm{ei}} & \mathrm{Hz} & 4.7 \cdot 10^{11} & 4.9 \cdot 10^{12} \\ v_{e 0} & \mathrm{~Hz} & 3.6 \cdot 10^{8} & 8.0 \cdot 10^{9} \\ v_{i 0} & \mathrm{~Hz} & 2.2 \cdot 10^{6} & 5.0 \cdot 10^{7}\end{array}$

It may be concluded, therefore, that $v_{i_{0}} \ll v_{e o} \ll v_{e i}$. Hence, because $0<\mathrm{n} / \mathrm{n}_{\mathrm{o}} \leq 50$, in general, we may omit $\mathrm{Eq} \cdot(21)$ from the system. Physically, the relative smaliness of the coefficients in Eq. (21), compared to those of the companion equations, means that the deviation of $v_{0}$ from the velocicy of the surrounding gas is small.

Likewise, we neglect $v_{\text {io }}$ henceforth. 
The matrix elements of $a_{i j}$ are defined in Eq. (4-19) where, for example

$$
a_{12}=-n_{0} \frac{1+2 T_{e} \frac{\partial X}{\partial T_{0}}}{T_{0}+2 X T_{e}}
$$

and where the decivatives of $x$ are biven explicitly by kqs. (4-20) and (4-21). We have defined $J=-J_{0}+J_{i}$. We a'sisume $T_{e}=T_{i}$ as before.

To gain an order uf magnitude estinate of the collision frequencies, let us use the values from Table I, from which the following numbers derive.

$\begin{array}{llll}T & k K & 16 & 20 \\ n & m^{-3} & 1.3 \cdot 10^{23} & 2.6 \cdot 10^{24} \\ n_{0} & m^{-3} & 1.8 \cdot 10^{23} & 4 \cdot 0 \cdot 10^{24} \\ \ell n \Lambda & 4.2 & 3.0 \\ v_{e i} & H z & 4.7 \cdot 10^{11} & 4.9 \cdot 10^{12} \\ v_{e 0} & H z & 3.6 \cdot 10^{8} & 8.0 \cdot 10^{9} \\ v_{i o} & H z & 2.2 \cdot 10^{6} & 5.0 \cdot 10^{7}\end{array}$

It may be concluded, therefore, that $v_{\text {io }} \ll v_{\text {eo }} \ll v_{e i}$. Hence, because $0<\mathrm{n} / \mathrm{n}_{\mathrm{o}} \leq 50$, in general, we nay onit kq. (2l) from the systen. thysically, the relative smallness of the coefficients in Eq. (2l), compared to those of the companion equations, means that the deviation of $v_{0}$ from the velocity of the surrounding gas is small.

Likewise, we neglect $v_{\text {io }}$ henceforth.

To summarize, we need the following functions, to be substituted in our system of Eqs. (20), (22), and (23). 
To summarize, we need the following functions, to be substituted in our system of Eqs. (20), (22), and (23).

$$
\begin{aligned}
& v_{\text {eo }}=2 \cdot 10^{-15} n_{0} \text {, as given in Chap. } 5 \text {; } \\
& v_{\text {io }}=\left(m_{e} / m_{i}\right)^{1 / 2} \cdot v_{\text {eo }} \simeq 0 \\
& v_{e i}=v_{e i}\left(n, k T_{e}\right) \text { from Chap. 5; } \\
& \mathrm{X}=\mathrm{X}\left(\mathrm{kT}_{\mathrm{e}}, \mathrm{kT}_{\mathrm{o}}\right) \text { from Chap. 4; } \\
& \mathrm{n}_{\mathrm{o}}=\mathrm{n}_{\mathrm{o}}\left(\mathrm{X}, \mathrm{kT} \mathrm{T}_{\mathrm{e}}, \mathrm{kT} \mathrm{T}_{\mathrm{o}}\right) \text { from Chap. 4; } \\
& \mathrm{n}=\mathrm{n}_{\mathrm{o}} \mathrm{X} \text { as in Chap. 4; } \\
& \mathrm{P}_{\mathrm{e}}, \mathrm{P}_{\mathrm{o}} \text { as given in Chap. } 10 \text { as function of } \mathrm{T}_{\mathrm{e}} \text {. } \\
& \text { One of the features of our system of ODE's is the large }
\end{aligned}
$$
magnitude of its coefficients. A very fast rate of change is prompted even by small deviations from the stationary set, as a consequence. For example, when verifying the stability, we observed that most ${ }^{\star}$ small deviations from the statisnary condition were corrected on a time scale of less than $v_{e}^{-1}$. This observation is only of numerical significance; physically, this violates one of our premises discussed in chap. 2 if one were to consider time scales on the order of vei or less. Numerically, the system behaves like an asymptotically stable one, thus implying the existence of at least one Liapunov function. The search for one is simple, because the entropy as well as the free energy are known to be good candidates. At the same time, one may close the system by that same function. In Chap. 9 we discuss the Helmholtz free energy in the Debye space's electrostatic field and on the macroscopic scale, for that purpose.

*except for small excursions of $\mathrm{kT}_{\mathrm{o}}$ whose characteristic times are $v_{\text {eo }}^{-1}$ 


\section{CHAPTEK 8}

S" TATIUNARY CONDITIUNS

(mks units)

The stationary subset of Eqs $\cdot(7-20),(7-22)$, and (7-23) yields the relationships

$$
\begin{aligned}
& J=e \frac{n}{2 m_{e} v_{1} i} p_{e}+p_{0} 1 / 2 ; \\
& E=\frac{1}{\epsilon} \frac{2 m_{e} v_{1} \cdot i}{n}\left(p_{e}+P_{0}, 1 / 2 .\right.
\end{aligned}
$$

We recognize the spitzer resistivity in the ratio b/J of Eqs. (1) and (2) .

From the discussion in chap. 6, we recall that $J$ is approximately constant over the arc cross section, $J \approx I / r^{2} \pi$, where $r$ is the are radius, $r$ const. The loss function $\mathrm{P}_{e}+\mathrm{P}_{\mathrm{o}}$ in gray body radiation is radius and temperature dependent, the radiated power being proportional to the surface area in addition to its $T$ dependency. We write the Eqs. (1) and (2) thus as functions of $I_{e}$ and $r$, replacing $J$. This leaves us with two equations in the unknowns $F, T_{e}$, and $r$. The system may be closed by the stationary condition in the free energy within a Debye space or, similarly, in macroscopic space, as mentioned in the chapter immediately preceding. 


\section{CHAPTER 9}

LAGRANGIAN FUNCTEON

(mks units)

Similar to the variation of the sum over quantum states in Chap. 4, we now minimize the variation of the potential energy in the classical system of particle-particle and particle-field interactions. We follow this procedure in our search for a Liapunov function, i.e., a function with a minimum at the equilibrium point. Longmire, in his $b_{0 o k}{ }^{2}(q \cdot v \cdot)$, gave inspiration for our formalism in Sec. 9.1.

\subsection{Interaction Free Energy (particle-particle) (esu)}

The work done against the interaction of the ion (electron) $\alpha$ against the Coulumb field of all the others in a Debye sphere is given by the derivative of the interaction ret eneriy $F_{\text {ei }}$

$$
\frac{\partial F_{e i}}{\partial e_{\alpha}}=\psi_{\alpha} .
$$

The average potential " $r$ around the wth ion (electron) is given by the Maxwell-Boltzmann statistics and Poisson's equation for the Couiomb field, i.e.,

$$
\begin{aligned}
v \psi(r) & =-\rho(r)=-\frac{1}{v} \sum_{\beta} e_{\beta} e^{-e_{\beta} \psi(r) / k \Gamma} \\
& =-\sum_{i} e_{i} n_{i} \exp \left[-e_{i} \psi(r) / k \tau\right] .
\end{aligned}
$$

Debye and Hückel assume $e \psi(r) \ll k T$ (thus keeping only the first term in the expansion of exp) and we know that the o-orifr term vanishes because of charge neutrality. Hence, 


$$
\nabla^{2} \psi(r)=\left(\frac{1}{k T} \sum_{i} e_{i}^{2} n_{i}\right) \psi(r)
$$

The integral of Eq. (1) is given by

$$
\psi(r)=\frac{A}{r} \exp (-\kappa r),
$$

where $k^{2}$ is the coefficient in the r.h.s. of $\mathrm{Eq} \cdot(1)$. The continuity condition of the electric displacement at $r=$ a determines the constant A, i.e.,

$$
-\left.\left.\frac{\partial \psi}{\partial r}\right|_{r=a} \frac{A}{r^{2}}(1+\kappa r) \exp (-\kappa r)\right|_{r=a}=\frac{e_{\alpha}}{a^{2}} \text {. }
$$

Hence, Eq. (2) may be rewritten with the boundary condition considered, i.e.,

$$
\psi(r)=\frac{e_{\alpha}^{2}}{r} \frac{\exp [-k(r-a)]}{1+k a}
$$

which yields $\psi \equiv \psi(a)=e_{\alpha}^{2} / a(1+\kappa a)$, where $\psi(a) \delta e_{\alpha}$ is tine work done by increasing the charge of the $\alpha$ th ion by $\delta_{2}$, and moving the charge against the Coulomb fields of the rest of the charges present in the Debye space. The interaction free energy is then,

$$
F_{e i}=\sum_{\alpha} \int_{0}^{1} \psi_{\alpha}(\lambda) e_{\alpha} d \lambda
$$


where $\|^{\prime \prime}(i)$ is the value $\psi_{\alpha}$ when all charges $e_{\alpha}, \epsilon_{\beta}, \ldots$ are replaced by $\lambda e_{a}, \lambda e_{3}, \ldots$. Hence,

$$
F_{e i}=-r_{x} n_{\alpha} e_{\alpha}^{2} k \int_{0}^{1} \frac{\lambda^{2} \mathrm{~d} \lambda}{1+\lambda \kappa a},
$$

as a comparison with Eq. (1) will verify. By integrating Eq. (3 and expanding for $k a<1$, one obtains

$$
F_{e i}=-\int_{\alpha} \frac{n_{\alpha} e_{\alpha}^{2}}{3} \times g(k z),
$$

with $g(x)=1-3 / 4 x+3 / 5 x^{2}+\ldots$ By neglecting all but the 0 -order term in Eq. (4), an expression for the free energy per unit volume is obtained, i.e.,

$$
F_{e i}=-\frac{1}{3}\left(\frac{1}{k T}\right)^{3 / 2}\left(\sum n_{\alpha} e_{\alpha}^{2}\right)^{3 / 2}
$$

We have to normalize this with respect to the gas kinetic partial pressure which is invariant under elastic encounters, i.e.,

$$
\frac{F_{e i}}{n k T}=\frac{2}{3} \pi^{l / 2}\left(\frac{n^{l / 3} e^{2}}{k T}\right)^{3 / 2} .
$$

Questioning the validity of the assumption just made, concerning $\mathrm{ka} \ll \mathrm{l}$, we obtain $\mathrm{Eq}$. (5) again from the following statistical argument. 
Let $N$ be the actual number of electrons in a Debye volume, and a be their averagc number, i.e.,

$$
z=n+1, v .
$$

The probability that there are $N$ electrons and $M$ ions is thus given by

$$
P_{C}(i, M)=\frac{a^{N+M}}{B ! M !} e^{-2 a}
$$

Equation (6) shows that $M-N=0$ on che avergge, as it must, for overall charge neatrality. The average square difference, i.e.,

$$
\overrightarrow{(m-n)^{2}}=\overrightarrow{m^{2}}+\overrightarrow{n^{2}} \cdot 2 \overrightarrow{m n}
$$

which is a formula for computing dispersion. Evidently, one may write

$$
\overrightarrow{M^{2}}=\overrightarrow{N^{2}}=e^{-a} \quad \sum_{n=0}^{\infty} \frac{N^{2} a}{N !}=e^{-a} \text { a } \frac{d}{d a} a \frac{d}{d a} ; \frac{a^{l i}}{l i !}=a(1+a)
$$

and

$$
\overrightarrow{M N}=\vec{M} \cdot \vec{N}=a^{2}
$$

Therefore, the mean square excess of ions over electrons in a Debye volume is given by

$$
\overrightarrow{(\mathrm{M}-\mathrm{N})^{2}}=2 \mathrm{a}
$$


The mean potential in a Debye cell is then at least

$$
F(N, M)=\frac{e^{2}}{2} \overline{(M-N)^{2}} / x
$$

Equation (7) has been written as if all the charges were concentrated on the surface of a spherical Debye cell, which yields the minimum potentiai energy. If one now averages $1 / r$ against $r^{2} d r$ over the Debye volume (as a parochial correction) one gains a factor $3 / 2$, i.e.,

$$
F(N, M)=\frac{3}{4} e^{2} \cdot \frac{2 a}{r}
$$

If we now insert in $\mathrm{Eq}$. (8) the Debye radius for $r$, equate $a=n \cdot(4 / 3) \pi r^{3}$, and normalize again over the partial pressure, then we get back Eq. (5) identically. Equation (5) exhibits a maximum w.r.t. the temperature, as plotted in Fig. 2. That maximum is an instable equilibrium.

\subsection{Field Free Energy (particle-external field)}

The potential energy of the interaction between the electrons and the external field is given by

$$
\mathrm{L}^{F}=(\mathrm{e} \phi-\overrightarrow{\mathrm{eA}} \cdot \overrightarrow{\mathrm{V}}) / \mathrm{kT}
$$

where the first term is the energy supplied by ohmic heating ( $\phi$ being the external electrostatic potential), which is converted to ionization energy or dissipated. Therefore, we consider only the second cerm in Eq. (9). For $J=$ const across the arc, one obtains 


$$
\overrightarrow{\Delta A} \cdot \vec{v}=\frac{e J}{e n}{ }_{2}^{r} B_{1} d r=\frac{1}{n}\left(\frac{I}{2 \pi r}\right)^{2},
$$

where $I$ is the total current, $I=\pi r^{2} \mathrm{~J}$. If we normalize Eq. (10) w.r.t. kT, as in kq. (9), we obtain

$$
\mathrm{eA} \cdot \dot{V}=\frac{1}{\mathrm{n}} \frac{\mathrm{I}}{2 \pi \mathrm{r}} !^{2}
$$

Our normalization w.r.t. k.T is the same as that w.r.t. nkT, which we usied in Eq. (5), if we consider that $\mathrm{F}_{\text {ei }}$ is an energy per unit volume. we recognize in Eq. (11) the external $\rho^{-1}$ as defined in plasina physics, $\mathrm{L}^{\mathrm{F}}: ?^{-1}$.

In Chap. 13, we will find the points where the two components of the macroscopic free energy, i.e., Eq. (5) in 9.1 and $\mathrm{Eq} \cdot(11)$ will be stationary.

In Fig. 2 are plotted $\mathrm{L}^{\prime}$ and $\mathrm{L}^{\mathrm{F}}$ from Eqs. (5) and (9), where it can be seen that for the pressure range 1 to 25 bar the instable maxima in $L^{\prime}$ are within $0.5 \mathrm{kK}$ of che stable minima in $\mathrm{L}^{\mathrm{F}}$. For the stationary arc, we have $\mathrm{T}_{e}=\mathrm{T}_{\mathrm{o}}=\mathrm{T}$, for which the minimum of the field free energy is given by the condition

$$
\frac{\mathrm{dI}^{\mathrm{F}}}{\mathrm{dkT}}=\frac{\mathrm{d}}{\mathrm{dkT}} \frac{H_{0}}{\mathrm{nkT}}\left(\frac{\mathrm{I}}{2 \pi \mathrm{r}}\right)^{2}=0 .
$$

We discuss in Chap. 13 the solution of Eq (12), presently confining ourselves to the observation that the naive assertion $\beta=1$, found in several publications on the electric arc, is wrong. 


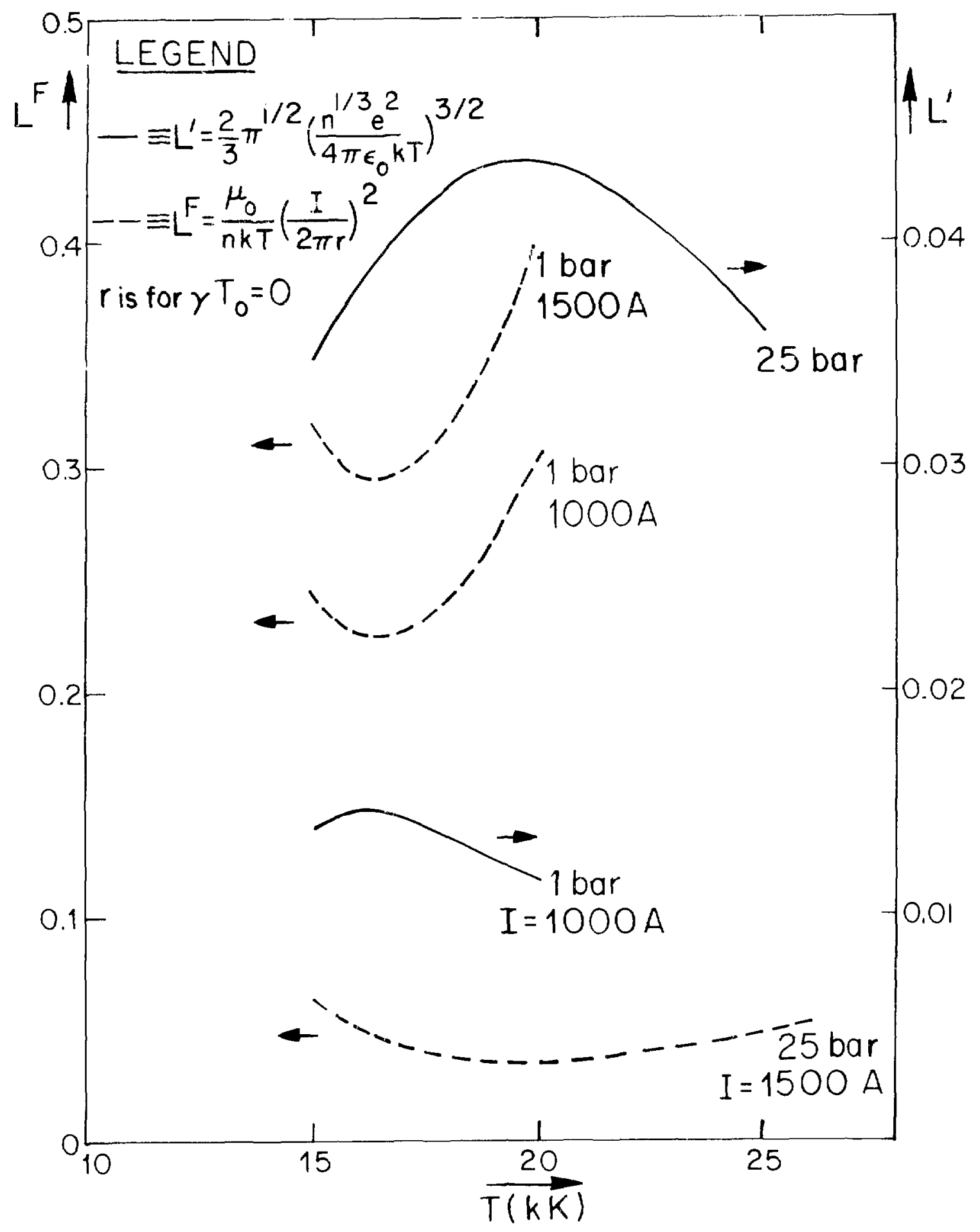

Fig. 2 Interaction free energy and field free energy vs temperature for $\mathrm{T}_{\mathrm{e}}=\mathrm{T}_{\mathrm{o}}=\mathrm{T}$ and $\gamma \mathrm{T}_{\mathrm{o}}=0$. 
CHAP'TER 10

RAIIATLVE: ENERCY TRANSPORT

(esi)

An electric are may be considered 22 to radiate like an opaque body with an absorption coefficient $\Gamma \simeq 0.14$ at $24 \mathrm{kK} ; 0.13$ at $20 \mathrm{kk}$; 0.09 at $15 \mathrm{kk}$. Follnwing are the balances in Planck's law.

If a body is assuned to be in equilibrium at temperature i with radiation inside an enclosure at this temperature, then the radiation power hw ${ }^{3} / \mathrm{c}^{2} \mathrm{f}(\mathrm{n})$ coss, is incident on an unit area of this body, per unit frequency and unit solid angle about the normal $k$ to its surface. A : raction a $(r)$ is absorbed, the rest of the radiation power reflected. The reverse process occurs with equal probability. The enitted power per unit area, in the frequency range $w$ to $\omega+d \omega$ is thus

$$
P_{e}(w) d(1)=i(u) \frac{2 \pi k\left(1,1^{3}\right.}{c^{2}} f(r) d(u) .
$$

Equilibrium considerations require that the mean power of the oscillators at temperature $T$ be given by a canonical distribution. The total power enitted per unit area of surface will be

$$
\left(P_{e}\right)_{t o t}=\int_{0}^{\infty} P_{e}(\omega) d \omega=\left(\frac{\pi^{2}}{60} \frac{k^{4}}{c^{2} h}\right) \quad a^{T} I^{4} \equiv \alpha T^{44}
$$

where the coefficient of aT is $5.7 \cdot 10^{-5}\left[\mathrm{erg} \mathrm{s} \mathrm{s}^{-1} \mathrm{cin}^{-2} \mathrm{~K}^{-4}\right]$, which is the Stefan-Boltzmann constant. We set $a=\Gamma$ to obtain the opaque body radiation power, so $a=1$ for black body radiation. Physically, $0<\Gamma<1$ means the black body property obtains over some parts of the frequency spectrum, transparency over the rest. 
CHAPTER 11

HEAT CONDUCTIVITY

(mks)

We estimate the heat conductivity of the monoatomic gas, using the hard core potential, and the heat conductivity due to electron thermal motion.

11.1 Atomic Nitrogen (esu)

Arcording to the Enskog-Chapman theory, the transport coefficients of a dense gas (cf. Chap. 9.3 in Hirschfelder, et al.'s book ${ }^{13}$ ) are given as functions of the dimensionless parameter $y=p \nabla / R T-1$, where $\nabla$ is the mol volume, and $R$ the universal gas constant. We get $g<0$ and therefore the low density limit applics, i.e.

$$
\lambda *=\frac{75}{64 \sqrt{\pi}} \frac{\sqrt{T^{\star}}}{\Omega(2,2)^{\star}} \frac{}{\left(T^{\star}\right)}
$$

(Hirschfelder 9.1-4́)

where $\lambda *$ is the reduced conductivity and $\mathrm{T} *$ the reduced temperature. The reduced quantities are defined as folluws

$$
\mathrm{T} * \equiv \mathrm{kT} / \varepsilon ; \lambda * \equiv \frac{\lambda \sigma^{2}}{\sqrt{\mathrm{m} \varepsilon}} \frac{\mathrm{m}}{\mathrm{k}}
$$

For air, we have a well depth $\varepsilon / k=87[\mathrm{k}]$, and a hard core radius $\sigma=3.3[\AA]$, so for our temperature range of 15 to $25 \mathrm{kK}$ we get $\Omega^{(2,2)^{*}}=1.0$ and $\lambda^{*}=8.7$ to 11.2 , resfectively. The corresponding conductivity values are $\lambda=2.5 \times 10^{4}$ to $3.2 \times 10^{4}\left[\mathrm{erg} \mathrm{s}^{-1} \mathrm{~cm}^{-1}\right.$ $\left.\mathrm{K}^{-1}\right]=2.5 . .3 .2[\mathrm{~mW} / \mathrm{cmK}]$ which are small, by an order of magnitude, compared with the electron heat conductivity. 
The effective coefficient of electron-ion thermal conductivity

$$
\lambda=\frac{\left(k T_{e}\right)^{5 / 2} k}{m_{e^{2}}^{l / 2} e^{4} \ln \lambda},
$$

which yields $0.3 \mathrm{~W} / \mathrm{kem}$ fur the Coulumb logarithen lnA $=4$ and $\mathrm{T}_{\mathrm{e}}=20 \mathrm{kK}$, with Spitzer's coefficient (cf. App. B-4).

\section{3 Enhancement of Thermai Conduct ion by Magnetohydrodynamic Turbulence}

A pressure balance may imply stability, such as that derived f rom

$$
\because P_{D}=\frac{1}{\mu_{C}}(\nabla \times \underset{B}{B}) \times \underline{B} .
$$

For ares of the type we are discussing, the manetic pressure is much smaller thari the gas peessure. We calculate, in Sec.9.2, the temperature for asymptotic stability. However, that calculation neylects the conditions at the boundary, that is at the arc surface. Actually, the plasma occupies only the finite region confined by a vacum magnetic field. our discussion, thus far confined to the inside of the plasma region, will, in the text immediately tollowing, be extended to the interface. We do this by the simplified assumption of a fluid film, that is a borentz gas, at the interface, cunducting the total current. Hence, recall the force balance (that is d'Alambert's prinicple applied at the fluid's outer surface), the continuity equation, the adiabatic condition, and Faraday's law, yielding the set 


$$
\begin{aligned}
& \rho \dot{\dot{\underline{v}}}=-\nabla p+\frac{1}{\mu_{0}}(\nabla \times \underset{\sim}{\underline{B}}) \times \underset{\sim}{\underline{B}} \\
& \frac{\partial \rho}{\partial t}+\nabla \cdot(\rho \underset{\sim}{\bar{v}})=0 \\
& \frac{d}{d t}\left(D^{-\gamma}\right)=0 \\
& \dot{\vec{B}}=\nabla \times(\underset{\sim}{\bar{v}} \times \underset{\sim}{b}),
\end{aligned}
$$

where $\gamma \equiv c_{v} / c_{p}=5 / 3$. In the usual manner, we get the second order OUE by differentiation and successive substitutions,

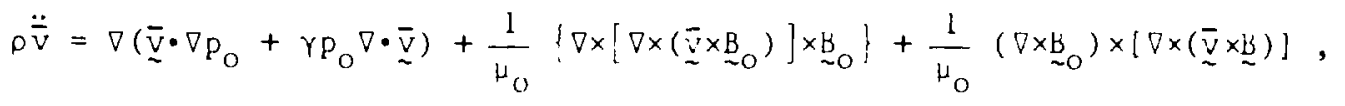

where $p_{O}$ and $B_{O}$ are defined at the outside surface of the current sheet, at which the terms enclosed in brackets[] are zero, similarly as $\nabla \mathrm{p}_{\mathrm{O}}$. Thus, only one term is left on the r.h.s. Integrating Eq. (7) yields, in our single-terin form,

$$
\rho_{0} \ddot{\xi}=\nabla \gamma \mathrm{P}_{\mathrm{O}} \cdot \nabla \cdot \xi=\gamma \mathrm{P}_{\mathrm{O}} \nabla^{2} \xi
$$

where $\xi_{z}=\underset{\sim}{x}-\underline{x}_{0}$ measures the departure, in configuration space, from the equilibrium at $\underset{\sim}{x}=\underline{\sim}_{0}$. One can separate $\left.\underline{z}_{\left(x_{0}\right.}, t\right)$ into a product of a space and a time-dependent part, $\xi=T(t) \cdot \xi\left(x_{0}\right)$, which yields $\ddot{\mathrm{T}}=-\omega_{\mathrm{k}}^{2} \mathrm{~T}$, and

$$
\rho_{o} \xi \omega^{2}=\gamma \rho_{O} \nabla^{2} \cdot \xi \cdot
$$


In cylindrical costáinales, to. (9), with

$$
\xi_{z}(r, t, z)=\xi_{z}(r) \in x_{1} \text { i }(n+t+k z)
$$

leads, for the z-component, ${ }_{2}$ to to bessel's equation

$$
\frac{d F}{d r^{2}}+\frac{1}{r} \frac{d f \cdot z}{d r}+x^{2}-\frac{\pi^{2}}{r^{2}} i r=0
$$

$w ! u \cdot r$

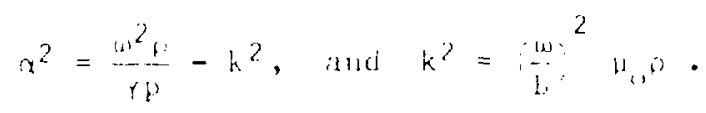

Obscrice tiat

$$
\frac{1}{Y P}<\frac{U_{O}}{B^{2}}
$$

because our $B \leq 0.5$.

$$
\text { With the b.c. } r=r_{0}+F_{r}=r_{0}+A / i k e^{i(m \phi+k z)} J_{m}^{\prime}\left(\alpha r_{0}\right) \text {, and }
$$

for the pinch instability with $m=0$, descriptively referred to as the sausage instability, the integral of $\mathrm{Fq}$. (10) becones 29

$$
-\frac{\omega^{2} r_{o} \rho}{2 p} \frac{J_{o}\left(\alpha r_{o}\right)}{J_{o}^{\prime}\left(\alpha r_{o}\right)}=1
$$


The solution values, $\omega^{2}$, of Eq. (11) are negative, that is $\omega^{2}<0$, indicating an instability, as expected. Using some numbers from Chap. 13, near the extremes shown in Figs. 4 and 5, we get

$\begin{array}{lllll}\mathrm{T}(\mathrm{kk}) & \mathrm{n}\left(\mathrm{cm}^{-3}\right) & \mathrm{r}_{0}(\mathrm{~cm}) & \mathrm{I}(\mathrm{A}) & |\omega|(\mathrm{rad} / \mathrm{s}) \\ 21 & 2.4 \cdot 10^{18} & 0.18 & 2000 & 12 \cdot 10^{6} \\ 18 & 0.34 \cdot 10^{18} & 0.28 & 2000 & 14 \cdot 10^{6}\end{array}$

The characteristic time for the sausate instability is therefore $\tau_{s}=0.2 \mu \mathrm{s}$.

Lindenth's two-dimensional calculations 30 show the sausage instability causes short, $T \ll T_{s}$, bursts of swirling motions of the plastia, startine at the boundary layer at intervals of a $\tau_{s}$ and convecing hot plasma to the surface. Uver time intervals $\tau \gg \tau_{s}$, one observes a plasma with vanishing radial temperature gradient.

From the $\mathrm{Eact}$ of $\mathrm{T}_{s}$ being the longest relaxation tine derives great significance in the dynamic arc: Phenomena evolving on the time scale of $1 \ldots 3 \mathrm{us}$, or shorter, are treaced inadequately in a onc-dimeusional formalism such as ours. Some heuristic approach is then sugrgested.

The plasma confined by a thin sideat', at surface current, that is the external $R=1$ condition, provides one of several possible preconditions for the $m=0$ instability. Fiat condition allows a siliple estinate of the characteristic lime for the sausage inscability, ds shown in Eqs. (3)-(11). An stability criterint must be that tor a plasma with $\beta>1$, nowever. lience, the kidontsev condition 30 obtains, that is

$$
-\frac{r}{p} \frac{d p}{d r}\left(\frac{b+5 B}{20}\right)<1
$$

which, if satisfied, insures stability, where $B$ is the local $\mathrm{n} \mathrm{kT} /(1 / 2$ $\mathrm{H}$ B). Because $-\Delta \mathrm{p} / \mathrm{p} \ll 1$, in our plasma, stability is provided by the 
wall confinement from the external gas pressure. As a precondition for the sausage instability still to develop, we expect $-\Delta p / p$ to become large when the local gas pressure $p$ apfroaches some small limit, as a result of overcooling by the turbulent removal oi heat. 


\section{CHAPTER 12}

TURBULENCE

(mks or esu)

The Navier-Stokes equation reduces to a form of the continuity equation if the axial variation of the drift velocity's radial component $\partial v_{r} / \partial z=0$. Thiel verified experimentally for a double-jet configuration typical for the electrodes of an air blast breaker that this condition is practically satisfied. Therefore, $\left|v_{z}\right| \propto z / L$ for $|z|<L$, and $\left|v_{z}\right|$ attains the speed of sound at $z=L$. According to Thiel's work, the turbulence in the discussed configuration is isotropic, i.e., $\int d \Omega \rho \vec{k}=0$, and, furthermore, that $\int_{0}^{\infty} d t \rho \vec{w}=0$, if $\vec{w}$ is defined as th. turbulent and $\vec{v}$ the drift component in the velocity. It follows that the mean enthalpy flow reduces to

$$
\overrightarrow{\mathrm{q}}_{\mathrm{T}}(\mathbf{r})=-\left(\rho \mathrm{w} \frac{\ell_{\mathrm{T}}}{3} \frac{\partial \mathrm{h}}{\partial \mathrm{T}}\right) \nabla \mathrm{T},
$$

where $\ell_{T}$ is the mixing distance over which turbulence transfers momentum in the radial direction. Thiel defines an equivalent turbulence-induced thermal conductivity $\kappa_{T}$ in the form of the expression in parentheses in Eq. (1) to obtain

$$
\overrightarrow{\mathrm{q}}_{\mathrm{T}}=\kappa_{\mathrm{T}} \nabla \mathrm{T}
$$

Radiative and turbulent energy transport generate a pronounced temperature gradient in the radial direction at the outer fringes of the arc, thus providing a flat temperatue profile in the interior. One can therefore write $\mathrm{Eq}$. (2) in the form 


$$
q_{T}=\frac{K_{T}}{\ell_{T}}\left(T-T_{e x t}\right)
$$

where $\mathrm{T}_{\text {ext }}$ is the external cooling medium's temperature. For the application in our equations, $\mathrm{k}_{\mathrm{T}} / \ell_{\mathrm{T}}$ is a single parameter to $\mathrm{I}$ it observation. 


\section{CHAPTER 13}

CLOSURE AND STUDY OF THE STEADY STATE

(mks units)

Considering the loss mechanisms discussed in Chaps. 10-12, we infer opaque body radiation and turbulence to be the most significant loss mechanisms. The heat flow per unit area at the arc surface, in this approximation, is given by

$$
P_{o}+P_{e}=\frac{2}{r}\left(\alpha T^{4}+\gamma^{T}\right)
$$

in the notations of Chaps. 10 and 12 . By substituting $\mathrm{J}=\mathrm{I} / \pi \mathrm{r}^{2}$ and Eq. (1), in Eq. $(8-1)$ we get

$$
r^{3}=\left(m_{e} / \pi^{2} e^{2}\right) \cdot \nu_{e i} I^{2} /\left[n\left(\alpha T_{e}^{4}+\gamma T_{o}\right)\right],
$$

where I is the current through the external circuit, and $v_{e i}=v_{e i}\left(k_{e}\right)$. $\mathrm{T}_{\mathrm{e}}$ and $\mathrm{T}_{\mathrm{o}}$ are determined by the relationships discussed in Chap. 9 and the text immediateiy following.

By substituting in Eq. (9-12) the Eqs. (13-1), (13-2), (5-4), and (5-5), one obtains a relationship for the minimum condition of the field free energy, corresponding to the minima in the plots for $L^{F}$ in Fig. 2, i.e.,

$$
\left(a_{12}+a_{21}\right)\left(\frac{1}{\ln \Lambda}-3\right) \frac{k T}{n}+2 \frac{1+4 \frac{\alpha}{\gamma} T^{3}}{a+\frac{\alpha}{\gamma} T^{3}}-\frac{3}{\ln \Lambda}=0 .
$$


We solve fig. (13-3) numerically to find the stationary temperature. Shorve that $\mathrm{T}$ in Fq. $(13-3)$ is a dependent of the transfer coefficient $y$ for the turbulent heat transport, discussed in chap. 12. Our system of equations is thus closed and we can calculate

- the are radius, irull Er. (13-2);

- the voltage gradient, trom Fq. (8-2); and

- the current density, from ta. $(y-1)$.

The two former quantities are plotted ws $y$ in Eib. 3, fur an oparue body absorption coefficient, $\Gamma=0.12$ (cf. Chap. 10).

In Fig. 4, we compare our results with the observations repurced by W. Hernann et al. 23 our basis for the comparison is:

a) The set of statir: pressure points, pstat, reported by Hermann ot ale, shown in Fi $\xi^{\prime} \cdot 4 a$;

b) Jur assumed linear increase in curbulent heat dissipation, increasing from 0.2 to $0.8 \mathrm{MW} / \mathrm{K} \mathrm{m}^{2}$ over the arc length $z=0 . \ldots 10 \mathrm{ctn}$;

c) $\Gamma=0.5$, which we infer Erom a paper of Thiel. 28

We obtain the values of 'I, $r$, and $E$ ploteed in Figs. 4b, $c$, and $d$, where the agreement between calculation and observation is best for the temperature, the difference between the two being less than $12 \%$. Observation and calculation disagree by a factor of 3 in arc radius and voltage gradient. This disagreement is caused mainly by our choice of $Y(\Gamma)$ and, to a lesser degree, by that of $\Gamma$. In using $\Gamma$ as a fitting parameter and calculating $Y$, with Thiel's formula given below, from Hermann et al.'s "outer cold gas velocity," $v$, and pressure, $p$, profiles, we obtain the agreement within a factor of less than 2 shown in Fig. 5, where $\Gamma=0.017$. Thiel's formula is given by

$$
Y=\frac{v}{c_{S}(T)} G(T)
$$

where $\mathrm{C}_{\mathrm{S}}(\mathrm{T})$ is the adiabatic speed of sound, and $\mathrm{G}(\mathrm{T})$ the turbulent heat flow potential plotted in Thiel'sl Fig. 32, p. 107. 

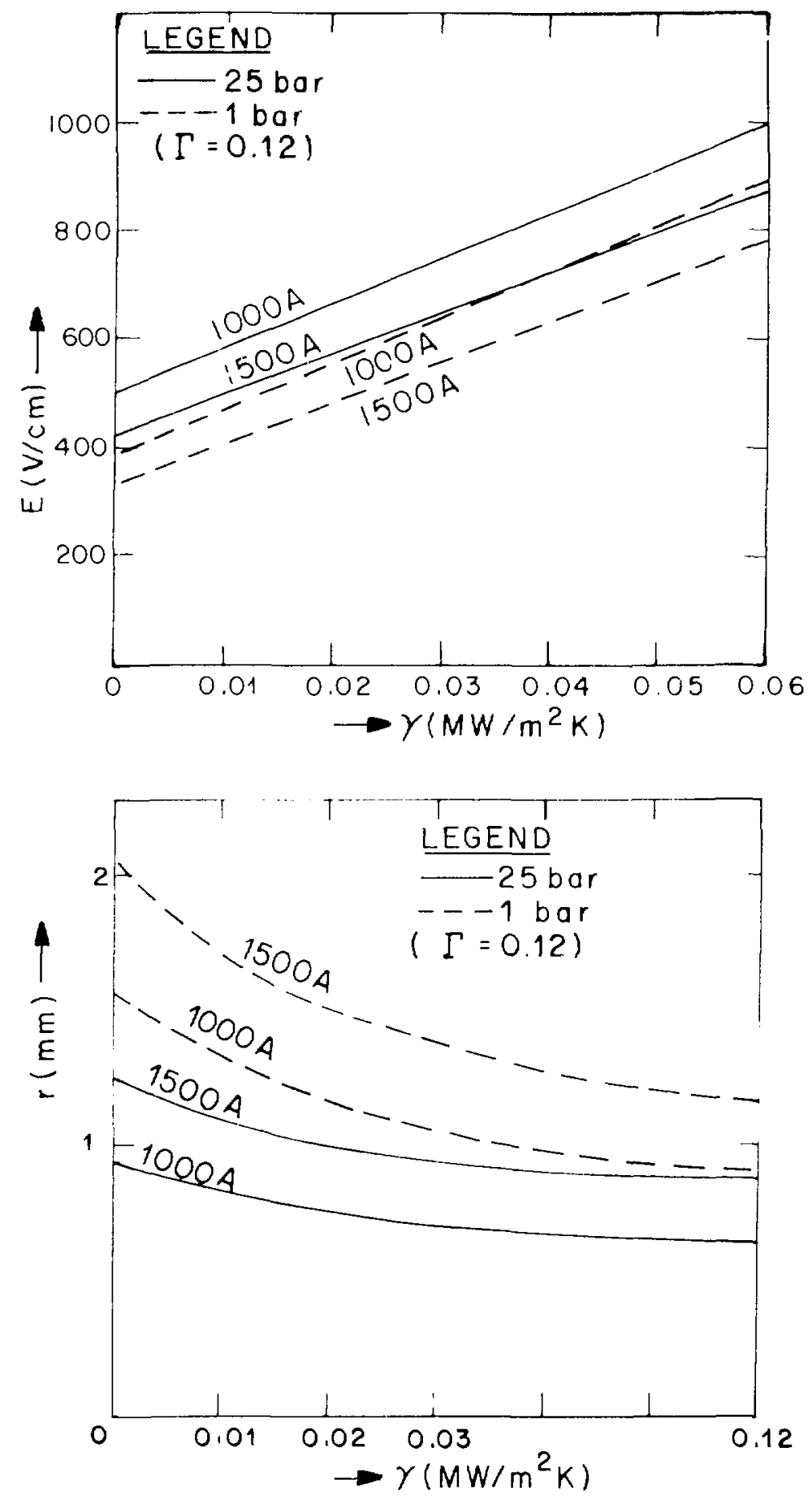

Fig. 3 Arc radius and voltage gradient vs turbulent teat transfer coefficient for ${ }^{r}=0.12$. 


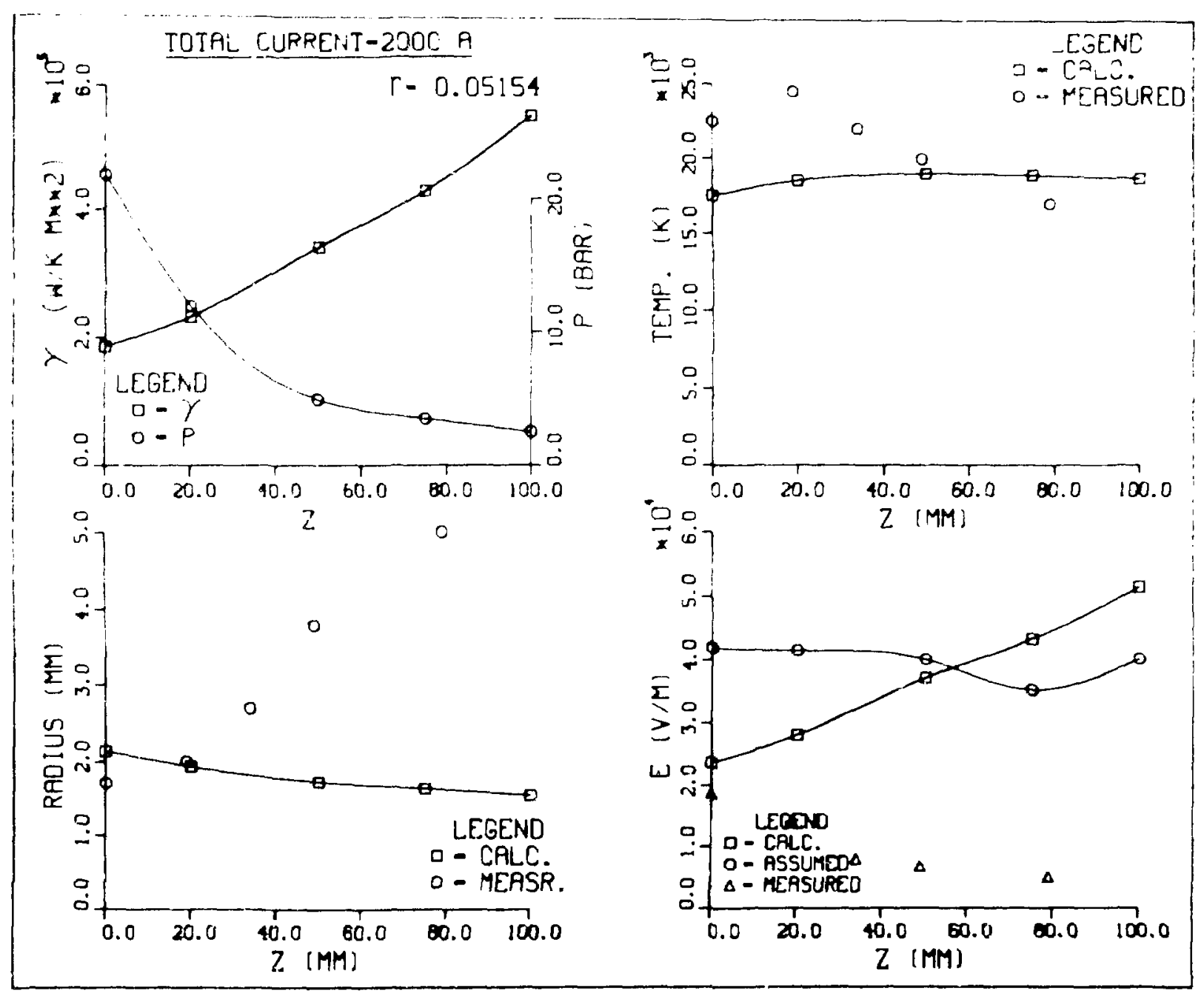

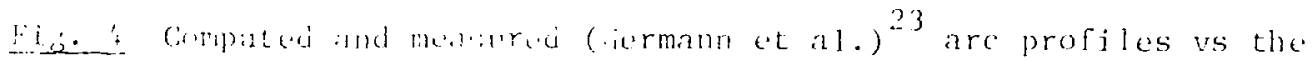
axial coordinate. The turbulent heat transfer coefficient, $\gamma$ is assumed to be a linear function of the culd gas velocity which, togeth*r with the prossure, P, profile, is that reported by Hermann et a 1. ; "dicjty, ", as reported by thiel. 28 


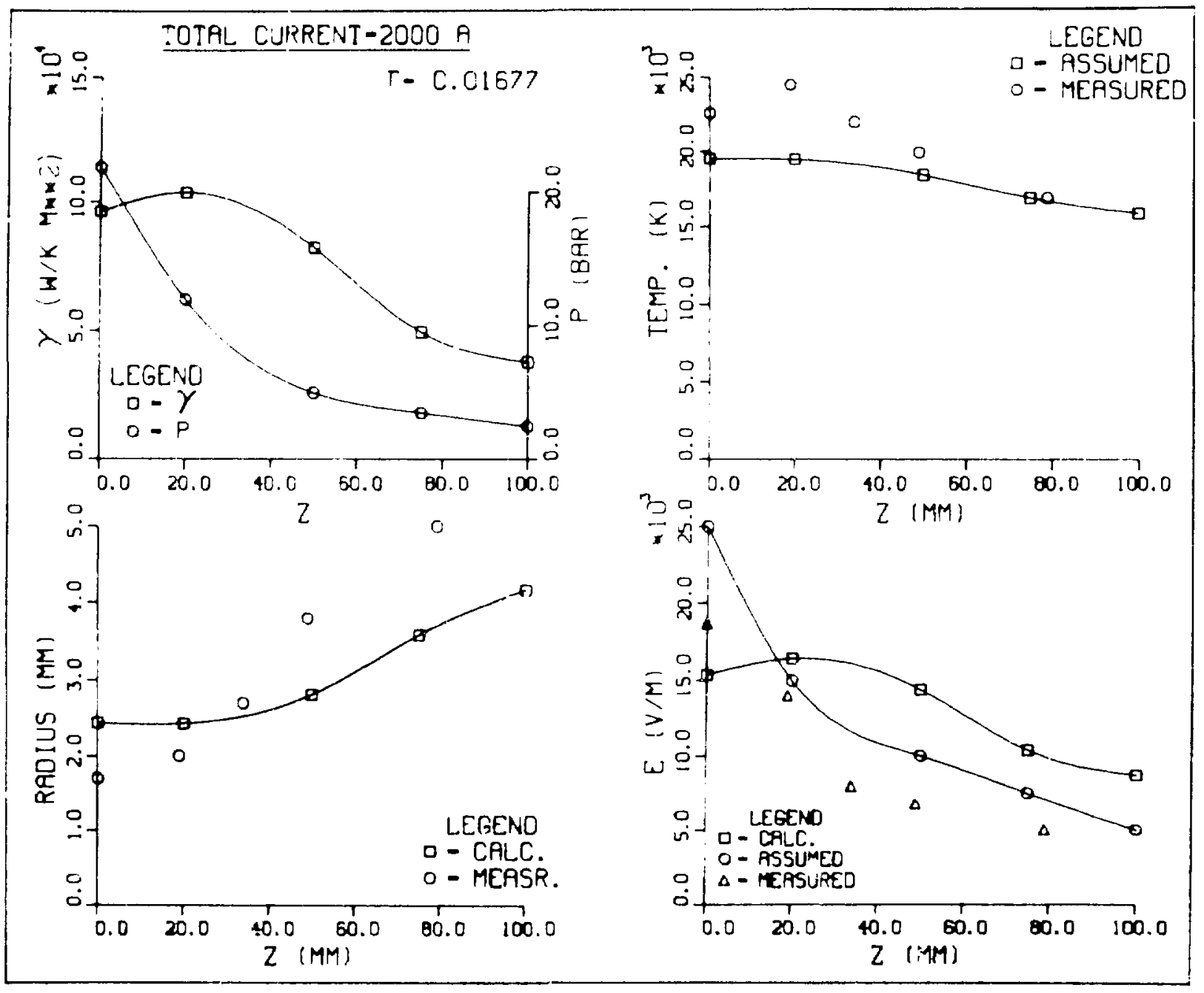

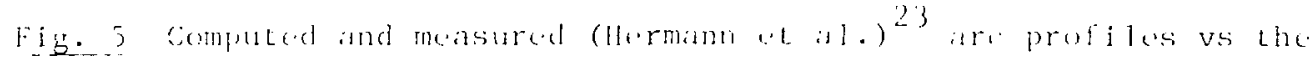

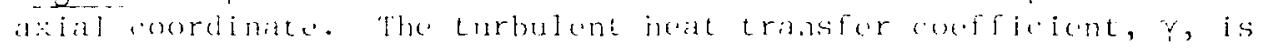
calculated from Hermann et al a s presisure and cold gals velocily pro-

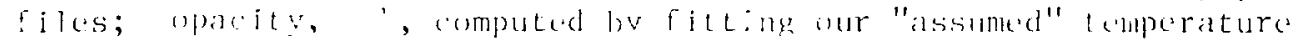
profile. 


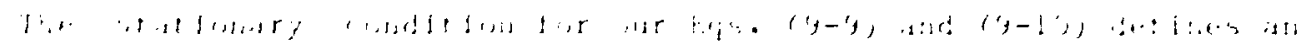

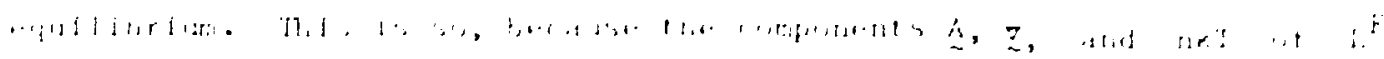

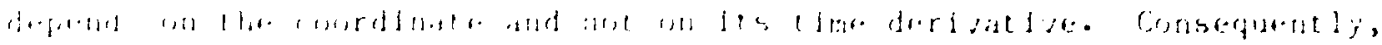

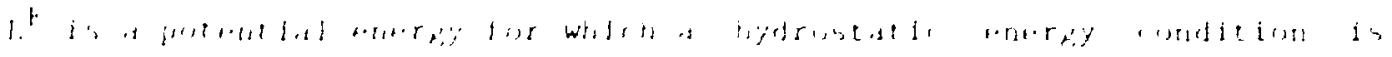

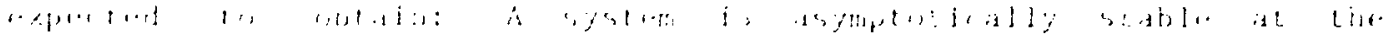

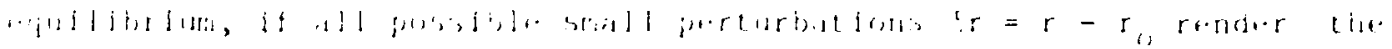

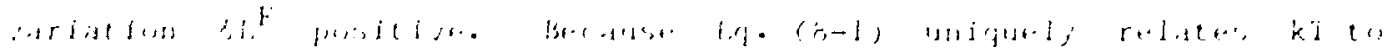

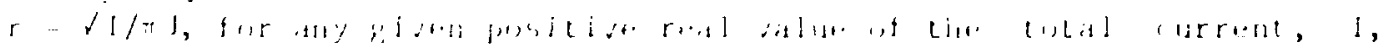

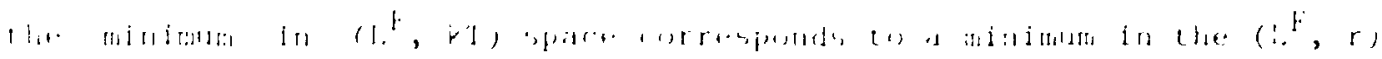

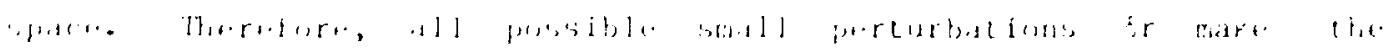

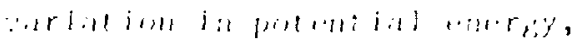

$$
\therefore 1 .=\frac{d w}{d r} ; r,
$$

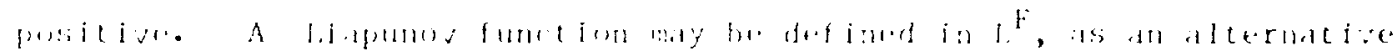
pront of the lisymptetr stability. 


$$
\begin{aligned}
& \text { Ghilititi.. }
\end{aligned}
$$

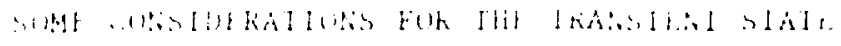

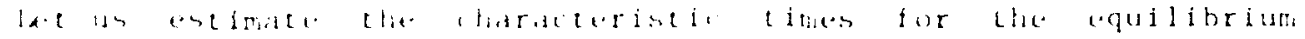

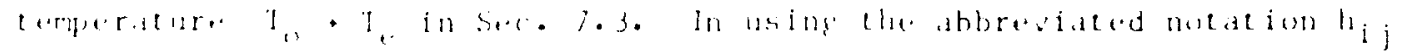

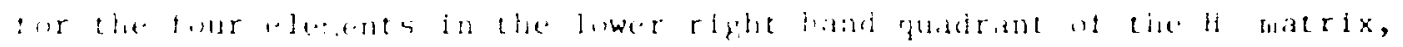

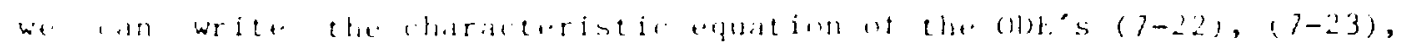

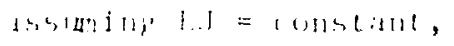

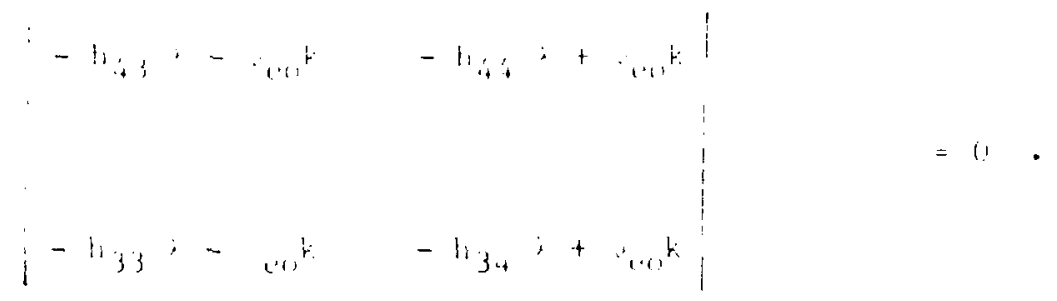

$$
\begin{aligned}
& \text { Henc., } \vdots 1=0 \text {, and } \\
& x_{2}=-\frac{h_{33}+n_{34}+h_{43}+h_{44}}{n_{34} h_{43}-h_{33} h_{44}}
\end{aligned}
$$

For the numbers in Table l, the raction part in $\mathrm{kg}$. (1) is one, within an order of magnitude. Hence, $i_{2} \sim-v_{e r}$, and the internal equilibrium is attained within the short times mat inned in Sec. 7.3.

During the transient state, $|\dot{I}|$ may be large, resulting in significant changes ot $r$ and $E$ over a short period of time. Consequently, one must take the buundary conditions into account.

The entropy and $\exp (-H)$, where $H$ is Boltzmann's $H-f u n c t i o n$, are additional Liapunov functions, which might, perhaps, contribute additional useful relationships when one considers the transient state. 
CHAPTLR 15

CUNCLUISLUNS

By the agreement between our calculated numbers and Herman et al.'s corresponding experimental data 23 of the arc axis temperature, we are led to infer a fairly flat temperature profile in the arc, most of the measured values being a few percent freater than our computed ones. In other words, if the calculated resslts predicted the correct average values (in the ari cruss section), and the measurements were a correct indication of the temperature on the axis, then our premise of the temperature gradient being small would be confirmed. It is the premise for simplifying the equations inmediately following $\mathrm{Eg} \cdot(7-19)$, as mentioned in the text supervening that equation. We have identified magnetohydrodynamir turbulence as the mechanism for generating the flat temperature protile.

From the cylindrical arc envelope function developed in Chap. 6, we infer a constant current density. Conversely, a fairly flat current profile is implied when tho deviations of the are from a right cylindrical genetry are as small as observed by Hermann et al. 23 as well as by others. The observed arc shape was approximately that of a truncated cone with a half angle of 5 to $6 \%$.

Historically, Steenbeck's intuitively stated minimum piinciple 25 has been discussed for several decades. It postulates an arc envelope described by a function $r=r(z)$ such that the arc voltage $\int t d z$ be minimized. Some observations have been known to confirm, other ones to disagree with this postulate. In departure, for the more fundamental reason to introduce an energy principle, we minimize the Helmholtz free energy on the microscopic and the macroscopic scales, and use the same functions to close the system of hydrodynamic equations.

The system of hydrodynamic equations $(7-20)-(7-23)$ is derived in Chaps. 3, 7, and Apps. B-C. Our formalism is based on the Enskog-Chapman approximation to derive the hydrodynamic equations from the Boltzmann equation. The method is presented explicitly in basically simple mathematics, not exceeding vector and tensor calculus. We have 
thus been able to clarify two Ealse assumptions found in the literature. One concerns the collision frequency, and the other is the frequently encountered assertion that the external $\beta=1$, which, if true, would be inconsistent with theory and observation.

Using the pressure and air velocity vs the axial coordinate data observed by Hermann et a1. ta test our equations, we calculate numbers for the voltage gradient, arc radius, and temperature, which seen to agree with the same investigators' experimental data, where we use two fitting parameters. These are the heat flow parameter for the turbulent transport, and the opacity. The former is a constant correlation factor between the air speed at the arc surface and thr turtulenc heat conductivity. Our numbers seem to indicate that these two are the dominant heat transport mechanisms.

Regarding the microscopic basis for our formalism, we have shown in Chap. 2 that the Boltzmann approximation is applicable to provide us

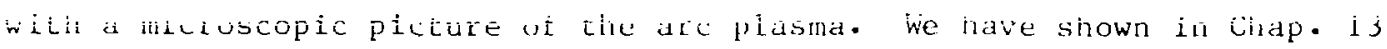
that the stationary condition is asymptotically stable, with characteistic times of typically less than a few tons of nanoseconds.

We have hus defined the complete set of equations that obtain in the arc's length element. The radius and axial voltage gradient are given as functions of the pressure in the adjoining gas, the heat dissipation coetficients $\Gamma$ and $\gamma$, and the total current. Because the inductance is a inction of the are radius $r$ and length $\ell$, the lat: derermined by the boundary conditions. In reiering to Fig. I I inductance element is given by

$$
d L=\mu_{0} \frac{d z}{4 \pi}\left(1+2 \ln \frac{R}{r}\right),
$$

where $r$ is given in $\mathrm{Eq} .(8-1)$ as function of $\gamma(z)$ and pressure $p=p(z)$. Hence, the total voltage over the arc length beccmes 


$$
v=\int_{0}^{l} E[i, p(z), Y(z)] d z+\frac{d i}{d t} \int_{0}^{l} d L+i \int_{0}^{l} \frac{d L}{d t}
$$

where the first term on the r.h.s. is the line integral of Eq. (8-2). For the simple configuration in Fig. Id, it is straightforward to use Eq. (2) for closing the set of external circuit equations. Usually, the first term is the only significant one on the right, in Eq. (2). In computing that term, the solving of Eq. (13-3) for $T$ is the most expensive part. Using Newton's method, our experience has been excellent if we provide the analytical derivatives, neglecting the terms with $\partial^{2} X / \partial T^{2}$ for which we compensate by scaling the Newton correction step, $\Delta \mathrm{T}$, to $1.3 \Delta \mathrm{T}$. The accounting time is thus aproximately $5 \mathrm{~ms}$ per evaluation of Eq. (13-8), on a CDC-7600 corputer.

we discuss, in Sec. 11.3, the $m=0$ magnetohydrodynamic instability, probably caused by piessure reduction from turbulent cooling of the arc surtace. That instability exhibits a characteristic time of approximately $0.5 \mu$ s and forces the radial convection of the heat dissipated by the turbulent gas. 


\section{CHAPTER 16}

\section{SUMMARY}

We show in Chap. 2 that the enormous electron and ion densities in an electric arc in air, at 10 to 50 atmospheres, imply a highly ionized plasma; and that the Boltzmann equation is the proper kinetic equation to be used. Our results agree with observation, even though we limit ourselves to considering a plasma with only three components, i.e., electrons, singly charged nitrogen ions, and nitrogen atoms. In Chap. 3, we derive, from the Boltzmann equation, the hydrodynamic equations for the three-component plasma, the equations right side being treated Eormally by simple functions of the collision frequencies among the species. The gas kinetic pressure exceeds the magnetic pressure by one to two orders of magnitnde, the magnetic pressure gradient, therefore, being negligible. In App. B, the collision frequency and thermal conductivity of the fully ionized plasma are derived by integrating the right side of the Boltzmann equation, by the normal method of Enskog and Chapman. Our results are compared with Spitzer's corresponding equations quoted in Chap. 5. The equations agree, except for the numerical coefficients, in which we differ by $6 \%$ in the collision frequency $\lambda_{e i}$, and by a factor 2.6 in the thermal conductivity $\lambda_{\text {ei }}$

Like all hydrodynamic equations, our set of equations is not closed and additional relationships are needed. Much of our work is devoted to obtaining closure. A few relationships are provided by the conditions of a minimum Helmholtz free energy, asymptotic stability of the equilibrium in each case provided. As the first of these asymptotically stable equilibria in our three-component plasma, we derive the Saha equation in Chap. 4, the gas kinetic total pressure being the invariant of the ionization process. In Chap. 9, we derive the free energy minimum in the Debye space (Sec. 9.1, q.v.) and show that minimum condition to be an instable equilibrium not useful for closure. In Sec. 9.2, we derive the free energy minimum for the particle-external field interactions, for which we can show asymptotic stability. Hence, 
the Saha equation and the equation defining the locus of the minimum particle-external field free energy are available for closure. From these, we get 15 to $23 \mathrm{kk}$, typically, for the arc temperature, and 0.2 to 0.6 for the degree of ionization. We find the temperatures of the three species to be nearly identical, thus implying the arc to be stationary. In Chaps. 7 and 8 , we show that the characteristic time for establishing the stationary condition is in a subnanosecond regime.

Additionit relationships for closure are provided by the boundary conditions. The first one of these is derived in Chap. 6, where we show that, for a given total current, the Lagrangian of a set of moving charges, in the magnetic and electric collective self-fields, is stationary, if the arc envelope function is related to the current density profile. As a useful example, a constant current density profile, i.c., one independent of the radial coordinate, requires a cylindrical are envelope. This is expected from observation also. In

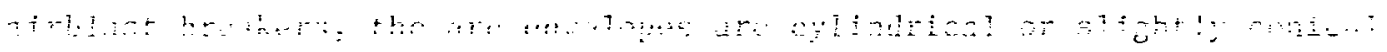
and the current density is nearly constant across the arc radius. A second relationship is provided by the Komtsev stability criterion at the arc surface, discussed in Sec. 11.3. The Komtsev condition indicates local instability for a low pressure or large pressure gradient. Because the pressure gradients are predominantly electromagnetic and small, compared with the $p / r$ ratio of the gas kinetic pressure over the arc radius, magnetohydrodynamic stability is provided by the gas pressure. Thus, we have wall confinement, except when the pressure is being reduced locally by overcooling from the forced removal of heat in the turbulent interaction between the high velocity gas and the arc surface. Overcooling may thus provide the starting condition for the $m=0$ sausage instability. We show, in Sec. 11.3, the sausage instability to evolve with a characteristic time of 0.5 us. Hence, because of mixing from the instability, a uniform radial distribution of current density and temperature can be expected, when averaged over a time long compared with the characteristic time of the magnetohydrodynamic instability. 
By the energy and boundary conditions, our set of hydrodynamic equations has thus been closed, for given thermal transport functions at the arc surface. As to the surface transport functions, we determine, in Chaps. 10 and 12, radiation and turbulence to be the predominant mechanisms. We introduce, in Chap. 10, the opacity $\Gamma$ as the characteristic coefficient for the radiative transport, and, in Chap. 12, the surface coefficient $\gamma=\kappa_{T} / l_{T}$ of the turbulent heat transfer, where $\ell_{T}$ is the mixing length, or turbulent layer thickness, over which the heat transfer is effecued, $\kappa_{T}$ being the turbulent thermal conductivity. We use $\Gamma$ and $\gamma$ as fitting parameters in Chap. 13, where we compute the axial profiles of the arc radius $r$, voltage gradient $E$, and temperature $\mathrm{T}$, assuming the axial profile of the gas pressure $\mathrm{p}$, and the two fitting parameters, $\Gamma$ and $\gamma$. We ubtain fair agreement with Hermann et al.'s reported data, as discu:sed in Chap. 13. We obtain agreement within a factor two, with Hermann it al.'s observed $E, r$, and

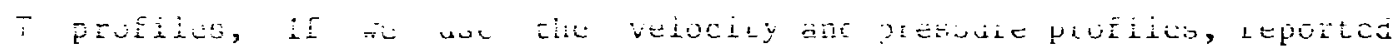
likewise in their paper, for calculating the profile of the turbulent heat transfer, $\gamma$, with a formula reported by Thiel, yielding $\gamma$ as $a$ function of the gas velocity and plasma surface temperature. We can thus show, in Chap. 13, that the accuracy in calculating the electric arc characteristics is limited by the available accuracy of the pressure and gas velocity profiles.

We have an additional relationship from the stationary condition of the Lagrangian, discussed in the text just preceeding, and in Chap. 6, relating the arc length to the arc radius, in Fqs. $(6,17)$ and $(6-18)$. These equations may be useful for closing the equation of the external circuit, in addition to our discussed variables $E$, $r$, and $T$. The usefulness of that additional relationship derives from the numerical insignificance of the are inductance in its interaction with the external circuit. We have not explored that global interaction between the arc and external circuit. 


\section{APPENDIX A}

CONVERSION FROM CGS TO MKS UNITS

Definitions

$$
F_{1}=k_{1} \frac{\mathrm{qq}^{\circ}}{\mathrm{r}^{2}} ; \quad E=k_{1} \frac{\mathrm{q}}{\mathrm{r}^{2}} ;
$$

Ampère:

$$
\begin{array}{r}
\frac{d F_{2}}{d l}=2 k_{2} \frac{I I^{\prime}}{d^{2}} ; \quad k_{3} B=k_{1}^{2} k_{2} \frac{I}{d} \\
\Delta \times E=-k_{3} \frac{\partial B}{\partial t} ; \quad \frac{1}{k_{2}}=c^{2}
\end{array}
$$

\begin{tabular}{|c|c|}
\hline Gaussian & for mks, replace Gauss' by \\
\hline$(E, \phi, V)$ & ${\sqrt{4 \pi \varepsilon_{0}}}(E, \phi, V)$ \\
\hline$(\rho, q, J, I, P)$ & $(\rho, q, J, I, P) / \sqrt{4 \pi \varepsilon_{O}}$ \\
\hline$(\mathrm{R}, \mathrm{L}, \mathrm{l} / \mathrm{C})$ & $4 \pi \varepsilon_{0}(R, L, 1 / C)$ \\
\hline$(\mathrm{H})$ & $\sqrt{4 \pi \mu_{O}}(H)$ \\
\hline (B) & $\sqrt{4 \pi / \mu_{0}}$ (B) \\
\hline (iv) & $\sqrt{4 \pi / \varepsilon_{0}}$ (D) \\
\hline c & $\left(\mu_{0} \varepsilon_{0} j^{-1 / 2}\right.$ \\
\hline
\end{tabular}

Faraday:

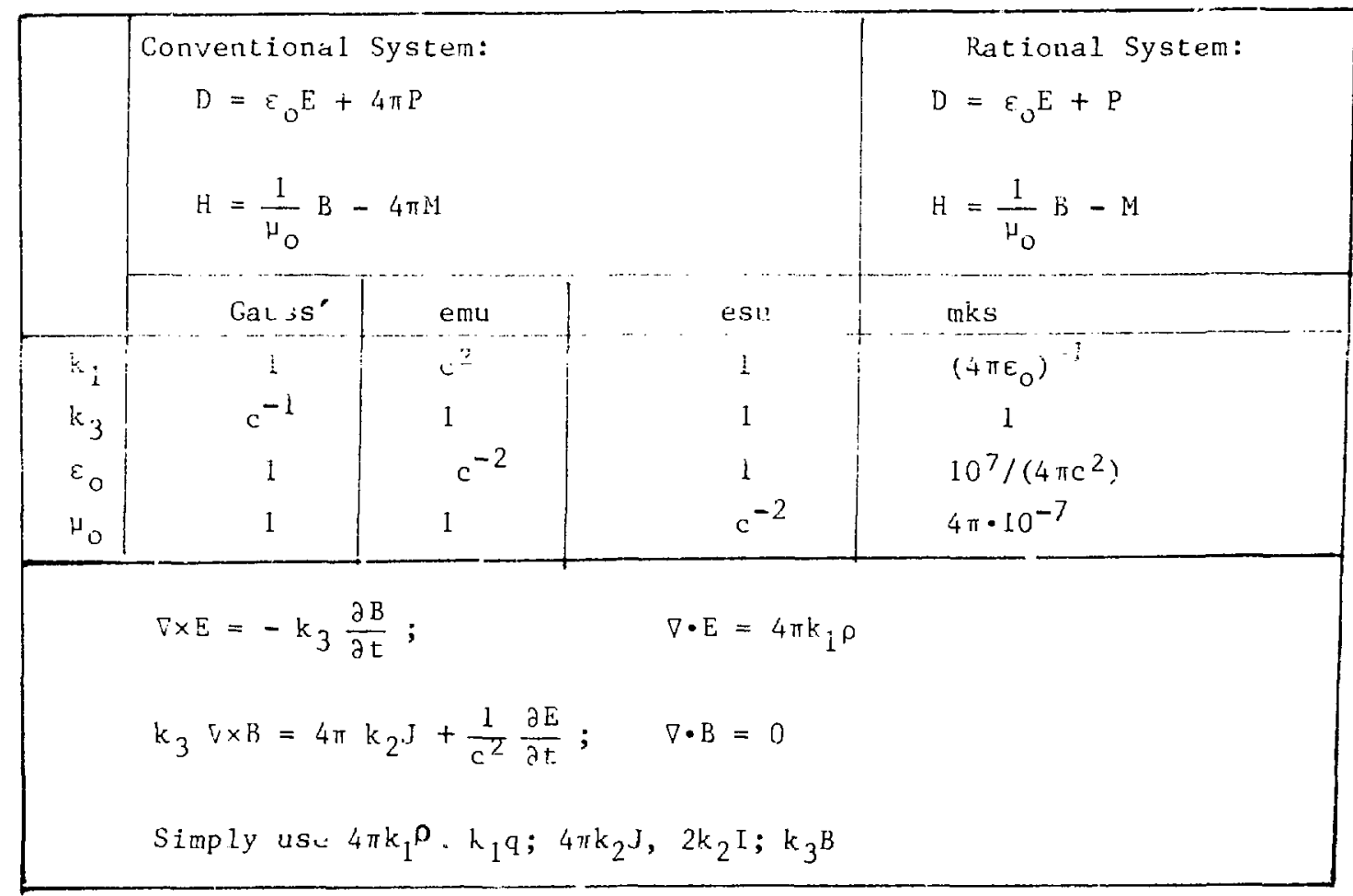

\begin{tabular}{|c|}
\hline cgs dimensions \\
\hline$E, D, E, B:$ \\
$g^{1 / 2 / \mathrm{cm}^{1 / 2} \mathrm{~s}}$ \\
\hline $\mathrm{I}: \mathrm{g}^{\mathrm{l} / 2} \mathrm{~cm}^{3 / 2} / \mathrm{s}^{2}$ \\
\hline $\mathrm{L}, \mathrm{C}: \mathrm{cm}$ \\
\hline $\mathrm{W}:$ erg $\equiv \mathrm{gcm}^{2 / \mathrm{s}^{2}}$ \\
\hline
\end{tabular}




\section{APPENDIX B}

ENSKOG-CHAPMAN METHOD FOR SOLVING

THE BOLTZMANN AND MAXWELI, TRANSPORT EQUATIONS

1. OUTLINE OF THE METHOD

It is sometimes convenient to write the Boltzmann equation in the simple notations of the streaming operator and the collision operator I, i.e.

$$
f=I(f f) \equiv \int d v_{\sim} \int d \operatorname{dog}\left[f^{\prime} f_{1}^{\prime}-f t_{1}\right] .
$$

where

$$
\mathscr{L}:=\frac{\partial f}{\partial t}+\underline{V} \cdot V_{r} f+\underset{\sim}{F} \cdot{\underset{\sim}{V}}_{\sim}^{f}
$$

If the system is not far from thermodyna.uic equilibrium, one may solve $\mathrm{Eq}$ - (1) by the method of successive approviacions in which the distribution function $f$ is expanded in powers of the parameter $\varepsilon$, i.e.

$$
f=f(0)+\varepsilon^{f(1)}+\varepsilon^{2} f(2)+\cdots,
$$

and where $\varepsilon$ measures the departure in density, from equilibrium. We simply redefine the velocity distribution by wricing the boltzmann equation (1) in the new form

$$
\delta f=\frac{I}{\varepsilon} I(f f) \text {. }
$$


Equation (4) has the appearance of the Boltzmann equation (1) for a velocity distribution of the form $f_{\text {formal }}=f / \varepsilon$. We proceed, therefore, by inserting the expanded veiocity distribution function (3) in Eq. (4) and equating the coefficients of the like powers of $\varepsilon$ :

$$
\begin{aligned}
& \varepsilon^{0}: 0=I\left[f^{(0)} f^{(0)}\right] ; \\
& E^{1}: \quad f^{(0)}=I\left[f^{(0)} f^{(1)}\right]+I\left[f^{(1)} f^{(0)}\right] ; \\
& \varepsilon^{2}: \quad f^{(1)}=I\left[f^{(1)} f^{(1)}\right]+I\left[f^{(0)} f^{(2)}\right]+I\left[f^{(2)} f^{(0)}\right] ; \quad(B-7)
\end{aligned}
$$

and so forth. The solution of $\mathrm{Eq} \cdot(5)$ is given by the equilibrium distribution

$$
\mathrm{f}^{(0)}(\underline{v})=\mathrm{n}^{(0)}\left(\frac{\mathrm{m}}{2 \pi \mathrm{kT}}\right)^{2 / 3} \mathrm{e}^{-\mathrm{mV^{2 }} / 2 \mathrm{kT}}
$$

Wher the system is close to equilibrium, we define $n=\int$ fdy; $\mathrm{n} \bar{v}=\int \underset{\sim}{v} \mathrm{f} d \underline{\sim} ; \quad \mathrm{n} \frac{3}{2} \mathrm{kT}=\int \frac{\mathrm{m}}{2} \mathrm{v}^{2} \mathrm{f}$ dv; which are slow.y varying functions of the arguments $(\underset{\sim}{r}, t)$. The following important postulate, which is prerequisite to the resolving procedure we are discussing, was contributed by Enskog. "Time does not enter expıicitly among the argivents of $\tilde{f}$ and $\partial \phi / \partial t$, but unly implicitly through $\bar{\Phi}$ and its spatial derivatives, where $\phi=\int \phi f d \underline{\text {. }}$ " When the system is not far from local

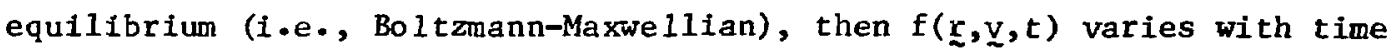
only through the functional dependence on $n(\underline{\underline{r}}, t), \underline{v},(\underline{\sim}, \underline{t}), T(\underline{r}, \underline{t})$ postulated by Enskog, i.e., $f(r, \underset{\sim}{v} \mid \mathrm{n}, \mathrm{v}, \mathrm{T})$; similarly for $f(\mathrm{~m})$ of all orders.* The procedure is, therefore, to insert the expansion $f(0)+$

\footnotetext{
* The physics reason for this is the fact that $n, y$, and $T$ are the privileged quantities that are invariant under binary collisions. A few collision intervals after the initial instant of the encounter, the time evolution is determined by our reduced set of variables.
} 
$\varepsilon f(1)+\ldots$ into the Boltzmann equation, the hydrodynamic equations, and into the relationships defining the thermal velocity (i.e., Enskog's "peculiar velocity") $\underline{V}=\underline{\mathrm{V}}-\overline{\mathrm{V}}$, the momentum flow tensor $\mathrm{R}=\mathrm{m} \int \underline{\mathrm{V}} \underset{\sim}{\mathrm{V}}$ $\mathrm{dv}$, and the heat $f l u x$ vector $\mathrm{g}=\int \mathrm{m} / 2 \mathrm{v}^{2} \underset{\mathrm{v}}{\mathrm{f}} \mathrm{dy}$. Solving the Boltzmann equation to the order $\varepsilon^{0}$, yields $f^{(0)}$. With this zeroth order distribution function, we calculate $\underset{\sim}{ }, \mathrm{R}$, and $\mathrm{g}$ for the zeroth order, i.e., $v^{(0)}, p^{(0)}, q^{(0)}$, and, insert them into the hydrodynamic equations for $n, \underline{v}$, and $T .$, With the latter quantities, we solve the Boltzmann equation to the order $\varepsilon^{1}$, yielding $f^{(1)}$. In principle, this procedure may be continued to orders of arbitrary degree. Thus, we follow Enskog in defining

$$
\begin{aligned}
& \underline{E}(\underline{r}, \underline{v}, t)=E\left(\underline{r}, \underline{v} \mid \Phi, \nabla_{r} \Phi, \ldots\right) \\
& \frac{\partial}{\partial t} \Phi(\underset{\sim}{r}, t)=\Psi\left(\underline{r}, \Phi, \nabla_{r}, \Phi, \ldots\right),
\end{aligned}
$$

where $\phi$ is any of the privileged dynamical (i.e., velocity-dependent) functions averaged over the velocity space (i.e., $n, \overrightarrow{m \underset{v}{v}, T}$ ). Since a power series has been adopted for $f$, the moments of $f, i . e ., \Phi$ and consequently $\Psi=\dot{\Phi}$, should now be written as a power series, as well:

$$
\begin{aligned}
& \Phi=\Phi^{(0)}+E \Phi^{(1)}+\ldots \\
& \Phi=\Psi^{(0)}+\varepsilon \Psi^{(1)}+\ldots
\end{aligned}
$$

The time derivative of the distribution function is obtained, in tensor formalism, by operating with the chain rule on Eq. (9), i•e.,

$$
\frac{\partial f}{\partial t}=\Psi \cdot \nabla_{\Phi^{f}}+\left(\nabla_{\mathrm{r}} \underset{\Psi}{ }\right):\left(\nabla_{\nabla_{\mathrm{r}} \Phi^{\mathrm{f}}}\right)+\cdots,
$$


where the higher order spatial derivatives are implied by the continuation dots in Eq. (9). The order of the tensors on both sides of the multiplication operators in Eq. (13) increases term by term; so must the multiplication operators, to be consistent with the chain rule. The following linear differential operator may thus be defined:

$$
\frac{\partial i}{\partial t}=\Psi(1) \cdot \nabla_{\Phi}+\left(\nabla_{r}{ }^{\Psi}{ }^{(i)}\right): \nabla_{\nabla_{r} \Phi}+\cdots
$$

Following Enskog's procedure, we substitute Eqs. (3) and (12) in Eq - (13) and write the resulting expansion in terms of the differential operator (14), i.e.,

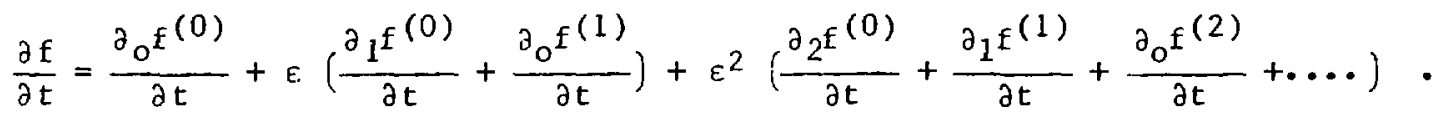

In this way, we have developed an expansion of the streaming term, $\Varangle^{\swarrow}$, of the Boltzmann equation (4) that may be written in the simple symbolic form

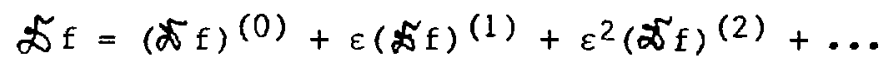

Hence, by comparing Eqs. (2) and (15) to Eq. (16), the identity

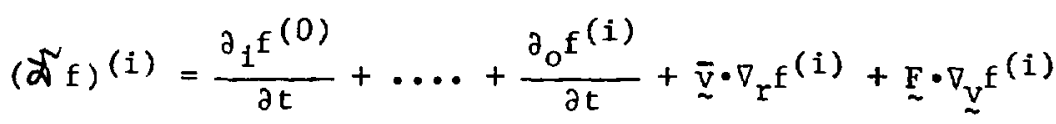


emerges for $i=0,1,2, \ldots$. Similarly, from Eqs. (5)-(6), we obtain for the collision integral, $I(f f)=\int d v_{1} \int d \Omega \sigma g\left[f^{\prime} f_{1}^{\prime}-f_{1}\right]$, the expansion

$$
I(f f)=I\left[f^{(0)_{f}}(0)\right]+\varepsilon\left[I\left(f(0)_{f}(1)\right]+I\left[f(1)_{f}(0)\right]+\ldots .\right.
$$

With both sides of the Boltzmann equation thus being expanded in powers of $\varepsilon$, we may write in detail the two lowest order integrals of $\mathrm{Eq} \cdot(18)$ by using the forms $f=f(0)\left[1+\varepsilon \Phi+\varepsilon^{2} \Psi+\ldots\right] ; \quad f(1)=f(0)$

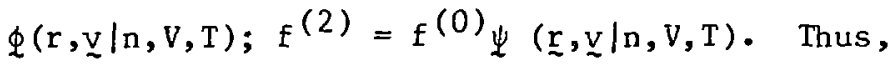

$$
I\left[f^{(0)_{f}(0)}\right] \equiv I(0)(1)=\iint d \underline{\sim}_{1} d \Omega \sigma g\left[f^{(0)}\left(\underline{v}^{\prime}\right) f^{(0)}\left(\underline{v}_{1}^{\prime}\right)-f^{(0)}(\underline{v}) f(0)\left(\underline{v}_{1}\right)\right]
$$

is the zeroth order integral, which vanishes as it should, agreeing with the result we obtained by comparing the cocfficients [cf. Eq. (5)] - The first order $\left(\varepsilon^{1}\right)$ integral becomes

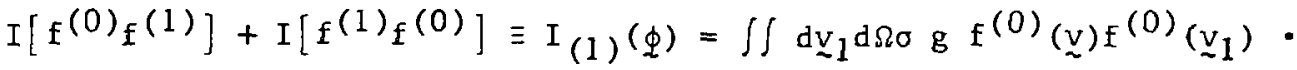

$$
\begin{aligned}
& \cdot\left[\phi\left(\underline{v}^{\prime}\right)+\phi(\underline{v})-\phi(\underline{v})-\phi\left(\left(\underline{v}_{1}\right)\right], \quad(B-20)\right.
\end{aligned}
$$

which is an integral over the unknown function $\phi$. Thus, we may use a general notation, such as $\Phi^{(r)}$, for the quantities $1, \Phi, \Psi, \ldots$; and denote the integral (2) by $I(r)\left[\phi^{(r)}\right]$. It will have been noticed that $r$ is used here to denote the order, not radius vector.

To determine the resolving method for our integral equation ( 1 ), one must first determine the type of the integral equation. Hilbert has shown for the case of a gas of rigid elastic spherical molecules, and Enskog for the general case, that $I(\phi)$ is an integral operator with a 
symmetrical kernel. To see this, we write the collision integral in terms of the collision cross section $\sigma$ and the angle $x$ enclosed by the trajectories of the colliding particles, i.e.,

$$
I(\phi)=\frac{1}{n^{2}} \iint f_{1} E\left(\phi+\phi_{1}-\phi^{\prime}-\phi_{1}^{\prime}\right)\left|\underset{\sim}{\underline{v}-\underline{v}_{1}}\right| \sigma \sin \chi d x d{\underset{\sim}{i}}_{1}
$$

The first term in parentheses, $\phi$, is independent of the variables of integration and the integral over it is written:

$$
K_{0}(v) \phi(\underline{v}),
$$

where $K_{0}(\underline{v})=n^{2}(m / 2 \pi k T)^{3} \iint d x d \underline{v}_{1} e^{-(m / 2 \pi k T)\left(v^{2}+v_{1}^{2}\right)} \cdot\left|\underline{v}-\underline{v}_{1}\right| \sigma$ sinx . The part of the integral (21) over the second term, $\phi_{1}$, may be expressed as

$$
\int K_{1}\left(\underline{v}_{\underline{v}}\right) \phi\left(\underline{v}_{1}\right) d \underline{\sim}_{1},
$$

and so forth, for the terms $\phi^{\prime}$ and $\phi_{l}^{\prime}$, leading to the Einal result

$$
n^{2} I_{(r)}\lfloor\phi(r)]=K_{0}(\underline{v})-\int K\left(\underline{v}_{\sim}, \underline{\sim}_{1}\right) \phi(r)\left(\underline{v}_{1}\right) d \underline{v}_{1},
$$

where $K\left(\underset{\sim}{v} \underline{v}_{1}\right)$ is a symmetrical function of its arguments, i.e., $K\left(v_{\sim}, \underline{v}_{1}\right)=k\left(\underline{v}_{1}, \underline{v}\right) . \quad A$ Fredholm equation is thus suggested, which we will discuss briefly below.

Let us first proceed with the topic of the successive approximations. To this end, it may be useful to summarize from Eqs. (4), (16), and (18) the formalism we developed thus far: 


$$
\begin{aligned}
& \left.\varepsilon^{0}: \quad \quad \mathrm{L}\left[\mathrm{f}^{0}\right)_{i}(0)\right]=0
\end{aligned}
$$

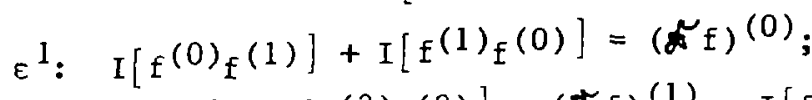

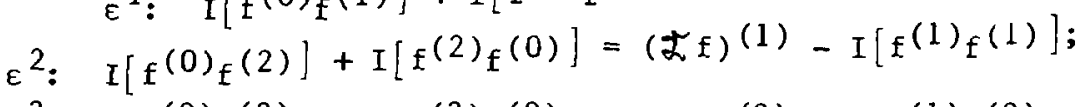

$$
\begin{aligned}
& \varepsilon^{3}: \quad\left[\left[\mathrm{f}^{(0)} \mathrm{f}^{(3)}\right]+\mathrm{I}\left[\mathrm{f}^{(3)} \mathrm{f}^{(0)}\right]=\left(\delta_{\mathrm{f}}\right)^{(2)}-\mathrm{I}\left[\mathrm{f}^{(1)_{\mathrm{f}}(2)}\right]-I\left[\tilde{f}^{(2)} \mathrm{f}^{(1)}\right]\right. \text {. }
\end{aligned}
$$

For every power of $\varepsilon$, the right side involves functions that are known from the previous equations of lower order. The unknown function appears only on the left and appears there linearly. It follows, that the solution of the set of integral equations (23), (24), .. is such that the macroscopic variables are always determined from $f(0)$ alone, i.e.,

$$
\begin{array}{ll}
\int \psi f(0) d \underline{y}=\int \psi f d \underline{v}=\bar{\psi}(\underline{r}, t) & \\
\int \psi f(r) d \underline{y}=0 & r=1,2, \ldots
\end{array}
$$

The unknowns are the functions $\Phi^{(r)} \equiv(1, \Phi, \psi, \ldots)$ in the integrals (22) which should be substituted in the 1.h.s. of Eqs. (23), (24), ... Inscead of rewriting Eqs. (23) and (24) again, let it suffice to say that they are inhomogeneous Fredholm integral equations of the second kind, whose general form is $\phi(z)+\lambda \int k\left(z, z_{0}\right) \psi\left(z_{0}\right) d z_{0}=\psi(z)$. Theory predicts a solution if and only if the right member is orthogonal to all solutions of the homogeneous equation. In attempting to solve the set of integral equations (23), (24), ... successively, we must satisfy, in each step, the orthogonality relationship

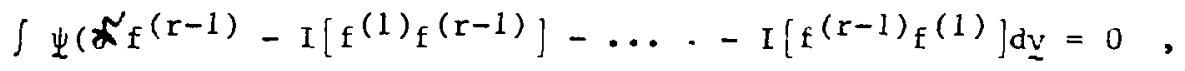

where $\psi$ is one of the summational invariants; these being the dynamical 
functions that are the solutions of the right members of Eqs. (23), (24), ... It follows that we have

$$
\int \Psi \mathcal{L F}^{(r-1)} d \underline{\underline{v}}=0
$$

rhich is the set of general conservation equations ( $\psi$ being the vector of the privileged functions), with the distribution function replaced by $f(r)$ of the $r-t h$ order. We may summarize by saying that the only nontrivial solutions admitted by the integral equations (23), and (24), ... are the dynamic functions $\Phi(v)=1, \underline{v}, v^{2}$ on considerations of conservation of momentum and energy in binary collisions, leading us to the conservation equations (27) ior $r=1,2,3, \ldots$.

\section{THE ZEROTH ORDER APPROXIMATION}

We find for $E^{\circ}$ :

$$
\begin{aligned}
& I_{(o)}=0 \text {; }
\end{aligned}
$$

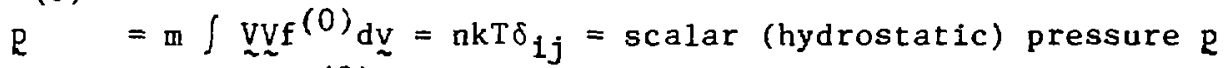

$$
\begin{aligned}
& \mathrm{q} \quad=\frac{\mathrm{m}}{2} \int \mathrm{v}^{2} \underset{\sim}{\mathrm{Vf}}(0)_{\mathrm{d}}=0 \text {. }
\end{aligned}
$$

Similarly, for the conservation equations*

* The general conservation equations are the equation of continuity for $\psi=m$; the equation of motion for $\psi=m \underline{v}$; and the equation of energy for $\psi=\mathrm{m} / 2 \mathrm{v}^{2}$ of a fluid, i.e.,

$$
\begin{aligned}
& \frac{1}{\rho} \frac{\mathrm{d} \rho}{\mathrm{dt}}=-\nabla_{r} \cdot \bar{v} \\
& \frac{1}{\rho} \frac{d \bar{v}}{d t}=\sum_{i} \rho_{i \sim i} F_{i}-\nabla_{r} \cdot R \\
& \rho \frac{d V^{2}}{d t}=-\nabla_{r} \cdot g-g: \nabla_{r} \bar{v}+\sum_{i} \rho_{i} F_{i} \cdot V_{i}
\end{aligned}
$$




$$
\begin{aligned}
& \psi=1: \quad \frac{\mathrm{dn}}{\mathrm{dt}}=-\mathrm{n} \nabla \underline{\mathrm{v}} \\
& \Psi=\mathrm{mV}: \quad \frac{\mathrm{d} \underset{v}{\mathrm{v}}}{\mathrm{dt}}=\underset{\sim}{\mathrm{F}}-\frac{1}{\rho} \nabla_{\mathrm{r}} \mathrm{P} \\
& \psi=\frac{m}{2} v^{2} \quad \frac{d T}{d t}=-\frac{2}{3} T \nabla_{r} \bar{v} .
\end{aligned}
$$

Equations (30)-(31) are Euler's hyc'rodynamic equations which are reversible in time, with adiabatic temperature changes, and with an equation of state which is the ideal gas law. The reversibility results simply from $I_{(0)}=0$.

3. TH: FIRST ORDER CORRECTION

If we substitute $f^{(1)}=f^{(0)} \phi$, as before, in Eq. (23); we get

$$
\mathrm{n}^{2} \mathrm{I}\left[\phi^{(1)}\right]=(\mathrm{f})^{(0)}
$$

Because $f^{(0)}$ is given as a function of $\underline{v}$, not $\underline{v}$, wr write the zeroth order streaming term

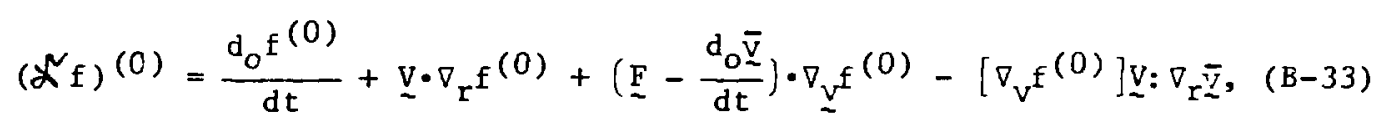

where $\underline{V}$ is regarded now as independent of $r$; and where

$$
\frac{d_{0}}{d t}=\frac{\partial}{\partial t}+\bar{v} \cdot \nabla_{r}
$$


By writing Eq. (33) in terms of $\ln f^{(0)}$ instead of $f(0)$, and substituting [from Eq. (8)], i.e.,

$$
\operatorname{lnf}(0)=\ln n-\frac{3}{2} \ln T-\mathrm{mV}^{2} / 2 \mathrm{kT}+\text { const, }
$$

we obtain

$$
\frac{{ }_{0} \ell n f}{d t}=\frac{1}{n} \frac{d_{o} n}{d t}+\left(\frac{m^{2}}{2 k T}-\frac{3}{2}\right) \frac{1}{T} \frac{d_{o} T}{d t} .
$$

By substituting into $\mathrm{Eq} \cdot(36)$ for $d_{0} \mathrm{n} / \mathrm{dt}$ and $\mathrm{d}_{\mathrm{O}} \mathrm{T} / \mathrm{dt}$ the corresponding right hand terms from the zeroth order conservation equations, we obtain

$$
\frac{d_{o} \ell n F(0)}{d t}=-\frac{m V^{2}}{2 k T} \nabla \cdot \bar{v} .
$$

Regarding the spatial and velority derivatives, we proceed in a similar fashion and obtain

$$
\begin{aligned}
& \nabla_{r} \operatorname{lnf}(0)=V_{r} \ln n+\left(\frac{\pi V^{2}}{2 k T}-\frac{3}{2}\right) \nabla \ell n T ; \\
& v_{v} \operatorname{lnf}(0)=-\frac{m}{k T} ! .
\end{aligned}
$$

With the substitutions defined by Eqs. (37)-(39), the first order Boltzmann equation becomes: 


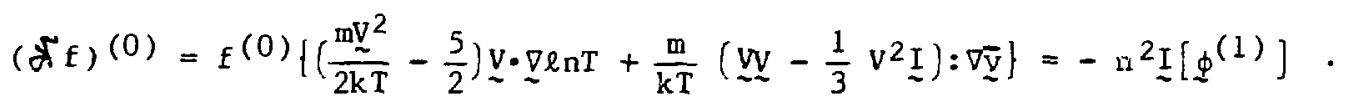

Equation (40) is one of our inhomogeneous Fredholm equations of the second kind for which the constraint $\int \psi f(0) d v=0$ provides uniqueness of the solution, as already discussed. This orthogonality condition is guaranteed exactly by the three zeroth order conservation equations. A unique solution exists therefore, and it appears from Eq ( 40 ) that it is a linear combination of the terms $\nabla \ell n T, \nabla \bar{Z}$, and a scalar function of $I\left(\phi^{(1)}\right)=0$. We can thus postulate $₹$ solution function, substitute it in the original Eq . (40), and solve for the parameter functions. Let

$$
\phi(1)=-\frac{1}{n} \stackrel{A}{\sim} \cdot \nabla \ell n T-\frac{1}{n} \underset{\sim}{B}: \nabla \overline{\underline{V}}+\underline{a}^{(1)} \cdot \Psi,
$$

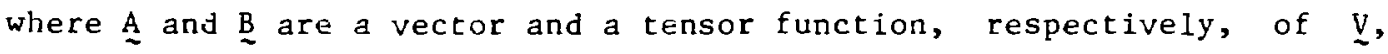
and $\underline{\alpha}^{(1)}$ is a constant vector. The components $\nabla \ell n T$ and $\nabla \underline{v}$ are linearly independent. By substituting Eq. (41) in (40), using the form (21) for the collision integral, we can identify the functions $\underset{\sim}{A}$ and $\underset{B}{-}$ by the following integral equations.

$$
\begin{aligned}
& n I(\underset{\sim}{A})=f^{(0)}\left(\frac{m V^{2}}{2 k T}-\frac{5}{2}\right) \underline{v} \\
& n I(\underset{\sim}{B})=\frac{m}{k T} f(0)\left(\underline{V}-\frac{1}{3} V^{2} I\right) .
\end{aligned}
$$

The pertinent orthogonality conditions may be written at once: 


$$
\begin{aligned}
& \int \Psi \mathrm{F}^{(0)}\left(\frac{\mathrm{mV^{2 }}}{2 \mathrm{kT}}-\frac{5}{2}\right) \underset{\sim}{\mathrm{V} d \underline{\mathrm{V}}=0} ; \\
& \int \Psi \mathrm{f}^{(0)}\left(\underset{\sim}{\mathrm{V}}-\frac{1}{3} \mathrm{~V}^{2} I\right) d \underline{\sim}=0 .
\end{aligned}
$$

Straightforward integration by parts shows that the integrals in Eqs. (44) and (45) indeed vanish identically, orthogonality thus being assured.

The vector constant $\alpha$ in Eq. (41) is fixed by the requirement (26) that

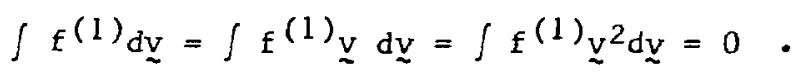

Because $\alpha$ is a vector, the equalities (46) imply $\underline{\alpha} \cdot \Psi \equiv c_{1}+c_{2} \underline{V}+c_{3} V^{2}=0$; consequently, $c_{1}=c_{2}=c_{3}=\underline{\alpha}=0$. By the same argument used in deriving Eq. (41) as a solution of Eq. (4), we can postulate the forms that $\underset{\sim}{A}$ and $\underset{\sim}{B}$ will have, to be solutions of the linear integral equations (42) and (43), i.e.,

$$
\begin{aligned}
& \stackrel{A}{A}=A(\underline{V}) \underline{V} ; \\
& \underline{B}=B(\underline{V})\left(\underline{V} \underline{V}-\frac{1}{3} V^{2} \underline{I}\right),
\end{aligned}
$$

where $A$ and $B$ are scalar functions of $n, T$, and $\underline{\mathrm{V}}$.

In pursuing our procedure of successive approximations, we will determine now the first order corrections to the momentum flow and heat flow tensors:

$$
\mathrm{e}^{(1)}=\int \mathbb{\mathrm { BVV }} \underline{\mathrm{f}}^{(1)} \mathrm{dy} \text {; }
$$




$$
q^{(1)}=\int \frac{\mathrm{m}}{2} \mathrm{v}^{2} \underset{\sim}{ }(1) \mathrm{d} \underset{\sim}{ } ;
$$

where $f^{(1)}=-1 / \mathrm{n} \mathrm{f}^{(0)}(\underset{\sim}{\mathrm{A}} \cdot \nabla \ln T+\underset{\sim}{\mathrm{B}}: \nabla \overline{\mathrm{V}})$ results froin $E q \cdot(41)$ with $\alpha=0$. Since integrals over odd powers of $\underset{v}{v}$ are zero, when integrated over the velocity space, the part in Eq. (49) contributed by A vanishes and we are left with

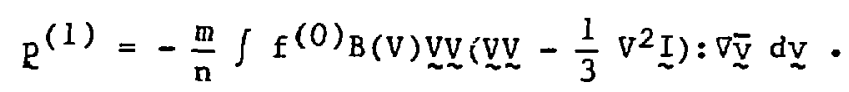

Since $\nabla \overline{\underline{V}}$ is a second rank tensor independent of $\underset{\sim}{\mathrm{V}}$, we may use the integral identity*

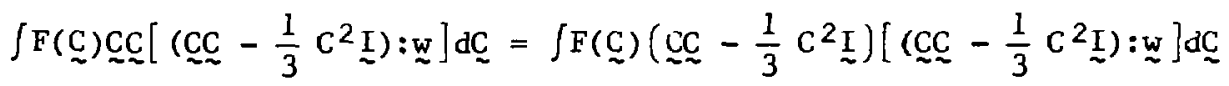

$$
\begin{aligned}
& =\frac{1}{5} \int F(\underset{\sim}{C})\left(\underset{\sim}{C}-\frac{1}{3} C^{2} I\right):\left(\underset{\sim}{C C}-\frac{1}{3} C^{2} \underset{\sim}{I}\right) d \underset{\sim}{S},
\end{aligned}
$$

where $\underset{\sim}{S}$ is the symmetric traceless (i.e., nondivergent symmetrical) part of $\underset{\sim}{w},\left(\underset{w}{w} \partial / \partial \underset{\sim}{\underline{r}} \underline{c}_{o}\right.$ in Chapman and Cowling's notation), i.e.,

$$
\underline{\sim}=\frac{1}{2}\left(\underset{\sim}{W}+{\underset{\sim}{w}}^{T}\right)-\frac{1}{3}(\underset{\sim}{w}: \underset{\sim}{I}) \underset{\sim}{I} .
$$

Thus,

\footnotetext{
${ }^{*}$ Chapman and Cowling Eq. $(1.421-i, i i, i v), 9$ where CC $-1 / 3 \mathrm{C}^{2} \mathrm{I}$ is the nondivergent tensor denoted (C C $)^{\circ}$.
} 


$$
R^{(1)}=-\frac{m}{5 n} \int f(0) E(\underline{V})\left(\underline{V}-\frac{1}{3} V^{2} \underset{\sim}{I}\right):\left(\underline{V}-\frac{1}{3} V^{2} \underline{I}\right) d \underline{\sim} \underset{S}{S},
$$

where

$$
S_{\alpha \beta}=\left(\frac{\partial \bar{v}_{\beta}}{\partial x_{\alpha}}+\frac{\partial \bar{v}_{\alpha}}{\partial x_{\beta}}\right)-\frac{1}{3} \nabla \cdot \bar{v} \delta_{\alpha \beta},
$$

which is the rate of shear tensor. ${ }^{*}$ The first order correction, $R^{(1)}$, to the pressure tensor is thus proportional to the rate of shear tensor. We may now substitute the original integral equation in Eq. (52) to obtain the simple expression

$$
\mathrm{P}^{(1)}=-\frac{1}{5} \mathrm{kT} \int \underset{\sim}{\mathrm{B}}: \underline{\sim}(\underset{\sim}{\mathrm{B}}) \mathrm{d} \underset{\sim}{ },
$$

which is a bracket integral $[\underset{\sim}{F}, G]=\int \underset{\sim}{G} \underset{\sim}{I}(F) d y$ and may be written in bracket form, i.e.,

$$
\mathrm{R}^{(1)}=-\frac{1}{5} \mathrm{kT}[\underset{\sim}{\mathrm{B}}, \stackrel{-}{\mathrm{B}}] \mathrm{S}^{* *} .
$$

Since Newton's law defines the pressure tensor

*cf. tensor $1 \cdot 33,2$ in Chapman and Cowling $\mathrm{p} \cdot 18$, also called "rate of
strain tensor". **In Ferziger and Kaper, $10, S$ has erroneously been omitted, cf. Eq. 5.4-35, p. 130 . 


$$
P=p \underset{\sim}{I}-2 n \underline{s}
$$

the coefficient of viscosity in the first crder Enskog theory simply follows from Eqs. (55) and (56), i.e.,

$$
\eta=\frac{1}{10} \mathrm{kT}[\underset{\sim}{\mathrm{B}}, \underset{\sim}{\mathrm{B}}]
$$

We use a similar procedure for calculating the heat flux vector whose definition is given by

$$
\mathrm{q}^{(1)}=\int \frac{m}{2} \mathrm{~V}^{2} \underline{\mathrm{V}} \mathrm{f}^{(1)} \mathrm{dV}
$$

We use, again, the first order correction to the distribution function given by $E q \cdot(41)$, with the constant $\underline{\alpha}^{(1)}=0$, which we just determined. Hence

$$
\mathrm{q}^{(1)}=-\frac{\mathrm{m}}{2 \mathrm{n}} \int \mathrm{f}(0) \mathrm{V}^{2} \underset{\sim}{\mathrm{V}}[\underset{\sim}{\mathrm{A}} \underset{\sim}{\mathrm{V} \ell \mathrm{n} T}+\underset{\sim}{\mathrm{B}}: \nabla \underset{\mathrm{V}}{\overline{\mathrm{V}}}] \mathrm{d} \underset{\sim}{\mathrm{V}} .
$$

Because $\underset{\sim}{\mathrm{B}}$ is a function of an even power of $\underset{\sim}{\mathrm{V}}$, which is multiplied by $\mathrm{V}^{2} \underset{\sim}{\mathrm{B}}$ does not contribute to the integral (59). We substitute $\mathrm{A}$ from $\mathrm{Eq}$ - (47) into Eq. (59) and make use of the integral identity

$$
\int F\left(\underset{\sim}{C)} \underset{\sim}{\mathrm{C}} \mathrm{dC}=\frac{1}{3} \int \mathrm{F}(\mathrm{C}) \mathrm{C}^{2} \mathrm{dC} \underset{\sim}{\mathrm{I}}\right. \text {, }
$$

by which Eq. (58) becomes 


$$
g^{(1)}=-\frac{m}{6 n} \int f^{(0)} V^{4} A(V) d v \nabla \ell n T
$$

Now, we add to Eq $\cdot(60)$ a zero-valued function of the expression - 5/2 $\mathrm{V}^{2}$, only to reduce Eq. (59) into one of the standard forms of the bracket integral. Such a zero function is the integral

$$
f(0) A(V) V^{2} d \underset{\sim}{v}=0,
$$

for reasons shown in the footnote. * Hence, we replace $\mathrm{V}^{4}$ in Eq. (60) by the expression $(2 \mathrm{kT} / \mathrm{m}) \mathrm{V}^{2}\left(\mathrm{~m} \mathrm{~V}^{2} / 2 \mathrm{kT}-5 / 2\right)$ to get

$$
g^{(1)}=-\frac{k T}{3 n} \int f^{(0)} V^{2} A(V)\left(\frac{m V^{2}}{2 k T}-\frac{5}{2}\right) d \underline{V} \nabla \ell n T .
$$

The scalar expression $V^{2} A(V)\left(\mathrm{m} \mathrm{V}^{2} / 2 \mathrm{kT}-5 / 2\right)$ in $E q \cdot(60)$ may be

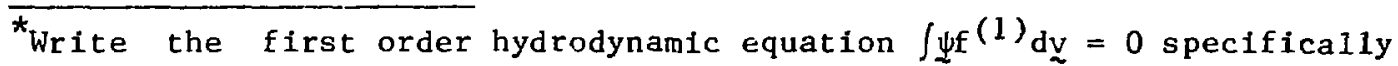
for the case $\Psi=\mathrm{mV}$ and with the general solution (distribution function $f^{(1)}=f^{(0)_{\phi}(1)}$ ) given in Eq. (4I):

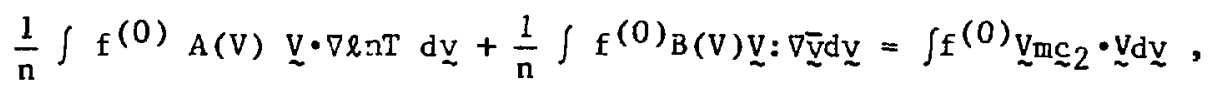

where $A(V)$ and $B(V)$ are given in Eqs. (47) and (48). The second term on the left is zero, because

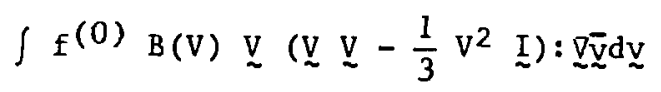

is a velocity integral over the velocity to an odd power. We are thus left with the following expression for the constant

$$
c_{2}=\frac{\frac{1}{3 n} \int f^{(0)} A(V) V^{2} d \underline{v}}{m \int f^{(0)} V^{2} d \underline{V}} \nabla \ell n T .
$$

It was pointed out in the comments on $E g_{(0)}(46)$ that $c_{2}=0$; hence, because $\int \mathrm{f}^{(0)} \mathrm{V}^{2} \mathrm{dv}=\mathrm{n} \mathrm{kT} j \mathrm{~m} \neq 0$, we have $\int \mathrm{f}(\dot{0}) \mathrm{A}(\mathrm{V}) \mathrm{V}^{2} \mathrm{dq} \stackrel{2}{=} 0$. 
replaced by $\underline{V} \cdot \underline{V}(\underline{V})\left(m V^{2} / 2 \mathrm{kT}-5 / 2\right)$ which, with Eqs. (47) and (42) becomes $A \cdot n I(A)$. It follows that $E q$. (61) may be written in the form

$$
q^{(1)}=-\frac{k T}{3} \underset{\sim}{ } \operatorname{nT}[\underset{\sim}{A}: \underset{\sim}{A}],
$$

(cf. Eq. (7.4.1, Chapman and Cowling).9 Thus, by Fourier's definition $q=-\lambda \underset{\sim}{\nabla} \mathrm{T}$, the thermal conductivity is given by

$$
\lambda=-\frac{k}{3}[\underset{\sim}{A}, \underset{\sim}{A}] .
$$

We have thus identffied $\mathrm{g}=-\lambda \nabla \mathrm{T}$, which is the heat flow vector in the first order Enskog Chapman theory.

The first order fluid dynamic equations are the Navier-Stokes equations of hydrodynamics, which are obtained by substituting into the general conservation equations the first order expressions for the pressure tensor and the heat flux vector:

$$
\begin{aligned}
& \frac{1}{\rho} \frac{d \rho}{d t}=-\underline{\nabla} \cdot \underline{V}, \\
& \rho \frac{d \bar{v}}{d t}=\rho \underline{F}-\nabla R+2 \eta \underline{\nabla} \cdot \underline{S}, \\
& \rho \frac{d T}{d t}=-\frac{2 m}{3 k}(-\underline{\nabla} \cdot \lambda \underline{\nabla T}+p \underline{\nabla} \cdot \underline{\bar{v}}-2 \eta \underline{S}: \underline{\nabla} \underline{\bar{v}}),
\end{aligned}
$$

where the rate of shear tensor is given by Eq • (53), and its gradient $\nabla \underset{\sim}{S}$ 1s, in vector notation, 


$$
2 n \underset{\sim}{\nabla} \cdot \underline{S} \equiv n \Delta \underset{\sim}{\bar{v}}+\left(\tau+\frac{1}{3} n\right) \nabla^{2} \overline{\underline{v}}
$$

or, in component notation

$$
(2 n \underset{\sim}{\nabla} \cdot \underset{\sim}{S})_{i}=\frac{\partial}{\partial x_{k}}\left[n\left(\frac{\partial \bar{v}_{i}}{\partial x_{k}}+\frac{\partial \bar{v}_{k}}{\partial x_{i}}-\frac{2}{3} \delta_{i k} \frac{\partial \bar{v}_{\ell}}{\partial x_{\ell}}\right)\right]+\frac{\partial}{\partial x_{i}}\left(\zeta \frac{\partial \bar{v}_{\ell}}{\partial x_{\ell}}\right),
$$

where the constants $n$ and $\zeta$ are called the coefficients of viscosity. For the incompressible fluid (i.e., for $\nabla_{\underline{v}}=0$ ), the last term vanishes. Recalling Boltzmann's $H$ theorem, the Navier-Stokes equations are irreversible, because $I_{(1)} \neq 0$.

To summarize, the problem of solving the Boltzmann equation has been reduced to solving the bracket integrals $[\underset{\sim}{A}, \underset{\sim}{A}]$, and $[\underset{\sim}{B}, \underset{\sim}{B}]$, which will now be discussed.

4. CALCULATION OF THE QUANTITIES A AND B

We demonstrated that the properties of the gas in the first order approximation are defined by the vector $\underset{\sim}{A}$ and tensor $\underset{\sim}{*}$ These quantities are determined by expressing them in the terms of Sonine polynomials $\mathrm{s}_{\mathrm{m}}^{(\mathbf{r})}$ ( $\mathrm{x}$; defined by the expression

$$
\left.(1-s)^{-m-1} e^{-x s /} \rightarrow\right)=\sum_{n=0}^{\infty} S^{n} s_{m}^{(n)}(x)
$$

N. J. Sonine (1880) defined these originally. They are related to the generajized Laguerre polynomials used by Schrödinger, both being orthonormal spherical functions. The Sonine polynomials are used in the kinetic theory of gases because of their rapid convergence for interaction potential functions of the type $1 / r^{5}$. In the special case of the $U(r) \propto r^{-5}$ potential function used by Maxwe11, the Sonine polynomials are eigenfunctions of the collision operator $I\left[\phi^{(1)}\right]$ and 
hence the exact solution for $\underset{\sim}{A}$ and $\underset{\sim}{B}$. The expansion (69) may be written explicitly for $s_{m}^{(r)}$ :

$$
S_{m}^{(n)}(x)=\sum_{p=0}^{n} \frac{\Gamma(m+n+1)}{(n-p) ! p ! \Gamma(m+p)}(-x)^{p} .
$$

Following are relationships which allow evaluating the bracket integrals.

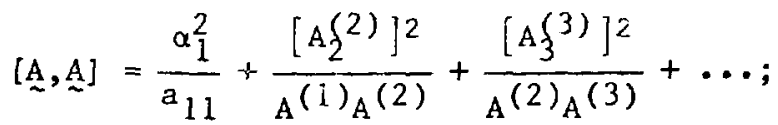

$$
\begin{aligned}
& {[\underset{\sim}{B}, \underset{\sim}{B}]=\frac{B_{1}^{2}}{b_{11}}+\frac{\left[B_{2}^{(2)}\right]^{2}}{B^{(1)_{B}(2)}}+\frac{\left[B_{3}^{(3)}\right]^{2}}{B^{(2)_{B}(3)}}}
\end{aligned}
$$

(cf. Eq. 7.51.12, Chapman and Cowling) ${ }^{9}$

$$
\begin{aligned}
& \alpha_{r}=-\frac{1}{n} \int f(0)\left(V^{2}-\frac{5}{2}\right) V \cdot s \frac{r}{3} / 2 V_{d} V_{;} \\
& B_{r}=-\frac{1}{n} \int f(0)(U V)^{0}:(V V)^{0} s_{5 / 2}^{(r-1)} d v ; \\
& \alpha_{1}=\frac{15}{4} ; \alpha_{2}=\alpha_{3}=\alpha_{4}=\ldots . .=0 ; \quad \beta_{1}=-5 ; \quad \beta_{2}=\beta_{3}=B_{4}=\ldots . .=0
\end{aligned}
$$

(cf. Eqs. (7.5i.5 and 6); 7.51.12, Chapman and Cowling) ${ }^{9}$

$$
\begin{aligned}
& a_{r s}=\left[s_{3 / 2}^{(r)}\left(V^{2}\right), s_{3 / 2}^{(s)}\left(V^{2}\right)\right] ; \\
& b_{r s}=\left[s_{5 / 2}^{(r-1)}\left(V^{2}\right)\left(V^{\prime}\right)^{\circ}, s_{5 / 2}^{(s-1)}\left(V^{2}\right)\left(V V^{\circ}\right]\right.
\end{aligned}
$$


(cf. Eq. 7.51.2 and 3, and Sect. 9.7, Chapman and Cowling)

$$
\begin{array}{ll}
A_{2}^{(2)}=-\alpha_{1} a_{21} & B_{2}(2)=-B_{1} b_{21} \\
A_{3}^{(2)}=-\alpha_{1}\left(a_{21} a_{32}-a_{31} a_{22}\right) & B(2)=-B_{1}\left(b_{21} b_{32}-b_{31} b_{22}\right. \\
A(1)=a_{11} \equiv \operatorname{det}^{(1)}\left(a_{i j}\right) & B(1)=b_{11} \equiv \operatorname{det}^{(1)}\left(b_{i j}\right) \\
A(2)=a_{11} a_{22}-a_{12}^{2} \equiv \operatorname{det}^{(2)}\left(a_{1 j}\right) & B(2)=\operatorname{det}^{(2)}\left(b_{i j}\right) \\
A(3)=\operatorname{det}^{(3)}\left(a_{i j}\right) & B(3)=\operatorname{det}^{(3)}\left(b_{i j}\right)
\end{array}
$$

(cf. Sects. 7.51 and 9.7, Chapman and Cowling)

$$
\begin{aligned}
& a_{11} \simeq 50 \Omega(1,1)-40 \Omega^{(1,2)}+8 \Omega^{(1,3)} ; \quad b_{00} \simeq 8 \Omega^{(2,2)}
\end{aligned}
$$

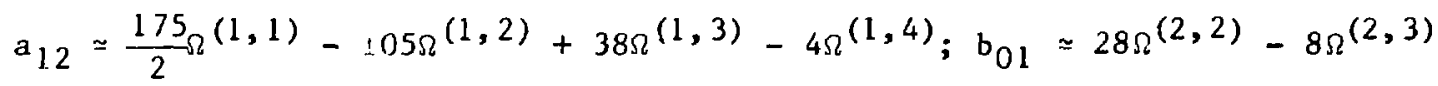

$$
\begin{aligned}
& a_{22} \simeq \frac{1225}{8}(1,1)-245 \Omega(1,2)+133 \Omega(1,3)- \\
& -28 \Omega^{(1,4)}+2 \Omega^{(1,5)} ; \quad b_{11} \simeq 98 \Omega^{(2,2)}-56 \Omega^{(2,3)}+8 \Omega^{(2,4)}
\end{aligned}
$$

(cf. Ferziger and Kaper: derived from Tables $7.3-7.5$ for $\mathrm{m}_{\mathrm{e}} / \mathrm{m}_{\mathbf{i}} \ll 1$; $\mu_{i}=m_{i} /\left(m_{i}+m_{j}\right) \simeq 0 ; \mu_{j}=m_{j} /\left(m_{i}+m_{j}\right) \simeq 1 ; i . e$. , for two-component gas mixture, Sect. 7.2 Ferziger a.Kaper) 10

$$
\Omega(\ell, \rho)=\frac{\pi^{1 / 2} A_{\ell}(s)(2 k T)^{\frac{s-5}{2(s-1)}} K_{12}^{2 /(s-1)} \Gamma\left(\rho+2-\frac{2}{s-1}\right)}{2 m^{1 / 2}}
$$


(cf. Eq. 10.3.10, Chapman and Cowling, 9 Eq. IV-102 (corrtzted)* Jance1 and Kahan, 11 where $A_{\ell}(s)=\int\left(1-\cos ^{\ell} x\right) u_{0} d u_{0}--c f$. Eq. 10.3.9, Chapman Cowling] 9 We have defined the interparticle force $F_{12}=K_{12} / r^{s}$. $A_{\ell}(s)$ is a number, and $x$ is the scattering angle, $x=2 \operatorname{arc} \sin \left[\left(1+u_{0}^{2}\right)^{-1 / 2}\right]$, where $u_{0}$ is the dimensionless number $u_{0} \equiv b\left(M_{r} g^{2} / K_{12}\right) l /(1-s)$, which is defined in binary collisions, where $b$ is the impact parameter, $g$ the relative speed, and $M_{r}$ the reduced mass (cf. Sect.9.1, Ferziger and Kaper, 10 Chap. 7, Jancel and Kahan). 11

For the inverse square (i.e., Coulomb interaction) law, $s=2$, we get $a_{12} / a_{11}=1.327, \quad a_{22} / a_{11}=2.082$, and $a_{11}=26.0 \pi^{1 / 2} A_{1}(2)$ $(2 \mathrm{kT})^{-3 / 2} \mathrm{e}^{4 / 2 \mathrm{~m}^{1 / 2}}$, and therefore,

$$
\lambda=\frac{2 k^{2} \mathrm{I}}{3 \mathrm{~m}}[\mathrm{~A}, \mathrm{~A}]=7.46 \frac{\mathrm{k}(\mathrm{kT})^{5 / 2}}{\mathrm{~m}^{1 / 2} \mathrm{e}^{4} \mathrm{~A}_{1}(2)} .
$$

For the collision integral, we get

$$
A_{1}(2)=\int_{1}^{b_{0}}(1-\cos x) u_{0} d u_{0}=\ln \left[1+\left(\frac{b_{0} g^{2} M_{r}}{e^{2}}\right)^{2}\right],
$$

where $M_{r} g^{2}=3 \mathrm{kT}$, and $b_{0}=\left(k T / 4 \pi n e^{2}\right)^{1 / 2}$ is the Debye length. Consequently, $A_{1}(2)=\ell n\left\{l+\left[3 / 2(k T)^{3 / 2 /(\pi n) 1 / 2} e^{3}\right]\right\}$, where we may neglect 1 by comparison to the expression in parentheses, thus reducing the expression to

$$
A_{1}(2) \simeq 2 \ln \left[\frac{3}{2} \frac{(k T)^{3 / 2}}{(\pi n)^{1 / 2} e^{3}}\right]=2 \ln \Lambda
$$

${ }^{*}(2 \mathrm{~m})^{1 / 2}$ should be $2 \mathrm{~m}^{1 / 2}$ as $\mathrm{gi}:$ en above. 
$\Lambda$ is the Coulomb cutoff parameter in Spitzer's definition.

To summarize chis, we obtain

$$
\left.\lambda=\frac{7.46}{2} k(k T)^{5 / 2 /(m 1 / 2} e^{4} \ln \Lambda\right),
$$

which is an expression in agreement witt. Spitzer's formula, except for the numerical factor, which is $20(2 / \pi)^{\hat{j} / 2} \equiv 10.16$ in Spitzer's formula, and 3.73 in our's.

To obtain an estimate of the coefficient of viscosity, i.e., $\ell=s=2$, we compute the bracket expression $[\underset{\sim}{B}, \underset{\sim}{B}]$ for a typical electron density $n$. Evidently, the integral $A_{2}(2)=\int_{0}^{\infty}\left(1-\cos ^{2} x\right) u_{0} d u_{0}$ is straightforward to evaluate if we replace, again, the lower limit by the hard-core cutoff parameter $b_{0 o}=e^{2 / 3} \mathrm{kI}$ given in Chap. 2 , and the upper limit by the Debye distance $b_{0}=\left(k T / 4 \pi n e^{2}\right)^{1 / 2}$. We define the dimensionless number $u_{0}=\operatorname{cotan}(x / 2)=b\left(M_{r_{g}}{ }^{2} / e^{2}\right)$, where $b$ is the impact parameter, with $b_{o o}<b=0$, and $\mathrm{M}_{\mathrm{r}^{2}}{ }^{2}=3 \mathrm{kT}$. For electrons with $\mathrm{n}=2.6 \cdot 10^{19} \mathrm{~cm}^{-3}$, we obtain $A_{2}(2)=5.4$. by evaluating only the term $B_{1}^{2} / b_{11}$ of the lowest order in the expansion for the bracket expression, we obtain $[\underset{\sim}{B}, \underset{\sim}{B}]=1.6 \cdot 10^{6} \mathrm{~s} / \mathrm{cm}^{3}$. We conclude that the viscosity term in Newton's law is negligitle. One may see this when Eq. (56) is written in the dimensionless form

$$
\frac{\mathrm{p}}{\mathrm{p}}=I+\frac{[\underset{\sim}{\mathrm{B}}, \underset{\sim}{\mathrm{B}}]}{5 \pi^{2} \mathrm{e}}\left(\frac{\partial \mathrm{J}_{\alpha}}{\partial \mathrm{x}_{B}}+\frac{\partial \mathrm{J}_{B}}{\partial \mathrm{x}_{\alpha}}\right) \text {, }
$$

where the second term on the r.h.s., with our numbers, becomes $7.5 \cdot 10^{-25}$ $\left(\partial J_{\alpha} / \partial x_{B}+\partial J_{B} / \partial x_{\alpha}\right)$, thus justifying our conclusion that we may equate the momentum flow tensor with the pressure tensor as follows 


$$
\mathrm{R}=\underline{I} \mathrm{P}=\underline{\mathrm{I}} \mathrm{n} \mathrm{kT} \text {. }
$$

5. SCATTERING CROSS SECTION

It is useful to relate the integral (71)--i.e., the quantity $A_{\ell}(s)$ introduced by Maxwe11--to the trajectory equations, because one may thus obtain the collision frequency in the first order theory. Suppose the polar angle $\theta$ be measured from the center of the stationary scatterer, as shown $i n \mathrm{Fig}$. $6 \mathrm{a}$. Let $\mathrm{M}_{r}, g$, and b denote, again, the reduced mass, relative velocity, and impact parameter, respectively. Conservation of angular momentum is then expressed by the relationship

$$
\mu \mathrm{r}^{2} \dot{\theta}=\mu \mathrm{gb} .
$$

If $U(r)$ denotes the interaction potential, then the conservation of energy in the binary collision process is expressed by

$$
\frac{1}{2} M_{r}\left(\dot{r}^{2}+r^{2} \dot{\theta}^{2}\right)+U(r)=\frac{1}{2} \mu g^{2}
$$

Autonomy from time is easily obtained, to form an equation for the $23 t h$, ie.,

$$
\frac{1}{2} M_{r}\left(\frac{g b}{r^{2}}\right)^{2}\left[\left(\frac{d r}{d \theta}\right)^{2}+r^{2}\right]=\frac{1}{2} M_{r} g^{2}-v(r) \text {. }
$$

Hence, we have the path integral

113 


$$
\theta=\int_{r}^{\infty} r^{-1}\left[\left(\frac{r}{b}\right)^{2}-\frac{2}{M_{r}} U(r)\left(\frac{r}{g b}\right)^{2}-1\right]^{-1 / 2} d r
$$

The angle $\theta=\theta_{m}$ for the point $r=r_{m}$ of closest approach is evidently obtained from the condition $\mathrm{dr} / \mathrm{d} \theta=0$, $1 . e$.

$$
\frac{d r}{d \theta}=0=r_{m}\left[\left(\frac{r_{m}}{b}\right)^{2}-\frac{2 U\left(r_{m}\right)}{M_{r}}\left(\frac{r_{m}}{g b}\right)^{2}-1\right]^{1 / 2},
$$

or,

$$
0=1-\frac{2 U\left(r_{m}\right)}{M_{r} g^{2}}-\left(\frac{b}{r_{m}}\right)^{2}
$$

It follows that the scattering angle $x=\pi-20_{m}$, shown in Fig. 6a, is given by

$$
x=\pi-2 \int_{r_{m}}^{\infty} r^{-1}\left[\left(\frac{r}{b}\right)^{2}-\frac{2 U(r)}{M_{r}}\left(\frac{r}{g b}\right)^{2}-1\right]^{-1 / 2} d r
$$

For an attractive Coulomb potential, one has

$$
\mathrm{U}(\mathrm{r})=-\frac{\mathrm{e}_{1} \mathrm{e}_{2}}{\mathrm{r}} \text {, }
$$

for which Eq. (72) becomes 

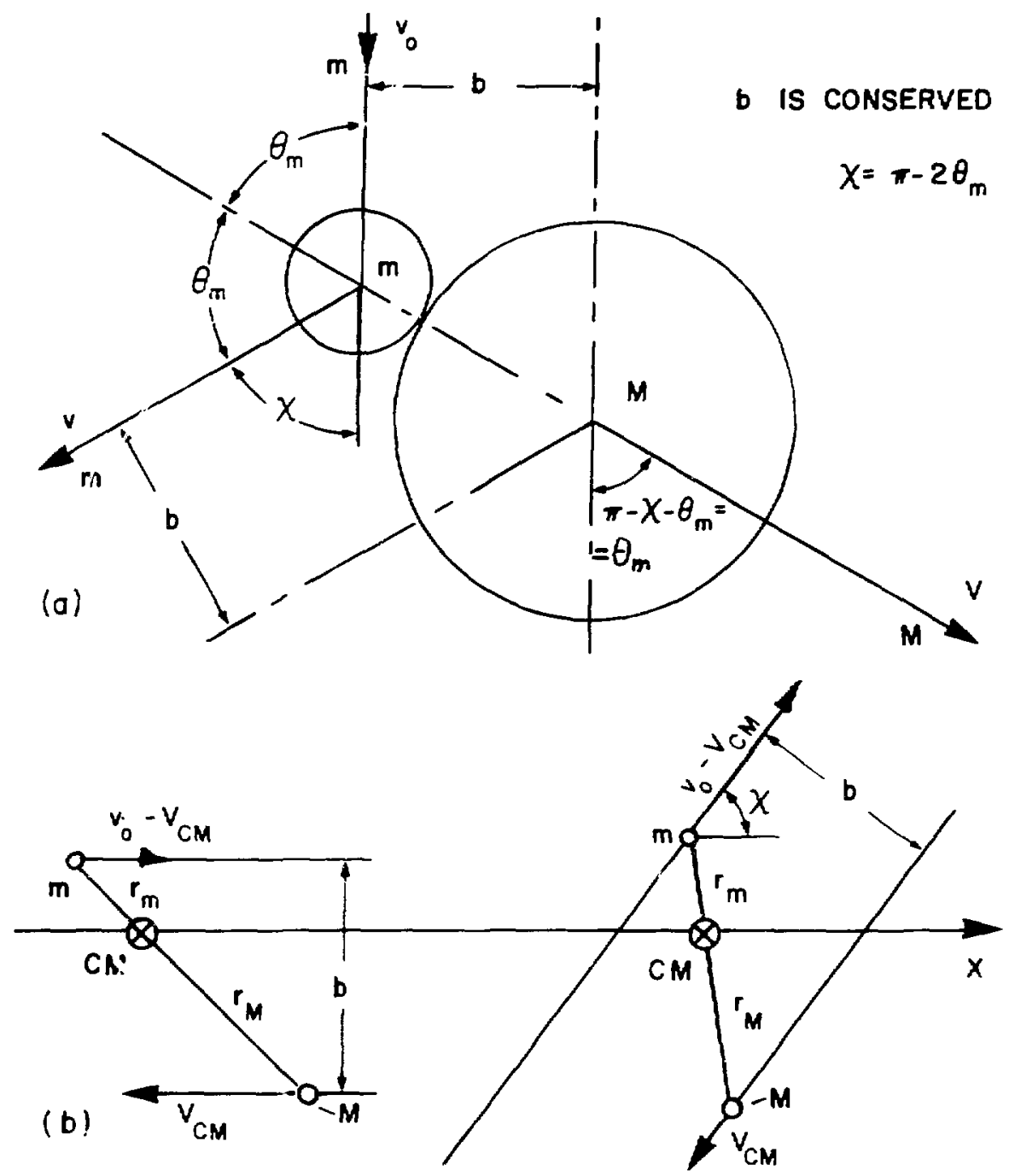

Fig. 6

(a) View of an elastic coliision in the laboratory, $M$ at rest initially

(b) Asymptotic views of an elastic collision in the Oi system:

$$
\mathrm{m}\left(\mathrm{v}_{\mathrm{o}}-\mathrm{v}_{\mathrm{cm}}\right)=\mathrm{MV}_{\mathrm{cm}}
$$

Loss of $x$-directed momentum component of $\mathrm{m}=\mathrm{m}\left(v_{\mathrm{o}}-\mathrm{v}_{\mathrm{cm}}\right)(1-\cos \lambda)$ 


$$
1-u_{m}^{2}-2\left(\frac{u_{m}}{u_{0}}\right)=0 \text {, }
$$

where

$$
u_{m} \equiv \frac{b}{r_{m}}, \quad u_{0} \equiv-b\left(\frac{M_{r} g^{2}}{e_{1} e_{2}}\right),
$$

and

$$
x=\pi-2 \int_{0}^{u_{m}}\left[1-u^{2}-2\left(\frac{u}{u_{0}}\right)\right]^{-1 / 2} d u ;
$$

thus yielding

$$
\cos x-\frac{u_{0}^{2}-1}{u_{0}^{2}+1}
$$

With the solid angle element being defined as $d \Omega=2 \pi \sin x d x$, we obtain an implicil relationship for the differential cross section, in the form of

$$
\mathrm{dn}=2 \pi \mathrm{Nb} d b \equiv 2 \pi N \sigma(x) \sin x d x
$$

From Eq. (74) follows directly, with Eq. (73), the Rutherford formula, i.e., 


$$
\sigma(x)=\frac{b}{\sin x} \frac{d b}{d x}=\frac{e^{2} e^{2}}{4 M^{2} g^{4} \sin ^{4} \frac{x}{2}}
$$

One may infer from Fig. $6 \mathrm{~b}$ that the momentum transfer cross section is given by

$$
Q^{(1)}=\frac{d n}{N}=2 \pi \int_{0}^{\pi} \sigma(x)(1-\cos x) \sin x d x=2 \pi \int_{0}^{\infty}(1-\cos x) b d b, \quad(B-75)
$$

because the quantity $M_{r} g \cdot(1-\cos x)$ represents the momentum loss in the initial direction of motion of a particle with the reduced mass $M_{r}$ and velocity $v=g \cdot *$ The cross sections, $\phi_{12}^{(\ell)}$, appearing in Chapman and Cowling's solution of the Boltzmann equation, are related as follows to our quantities $Q^{(1)}$ and $Q^{(2)}$ :

$$
\phi_{12}^{(\ell)}=\frac{g}{2 \pi} Q^{(\ell)},
$$

where the subscripts refer to the particles of type 1 and 2 . Hence, we have

* The v-directed momentum loss with respect to the center of mass velocity $\underline{v}_{c m}$ is obviously given by $m\left(\underline{v}-\underline{V}_{c m}\right)(1-\cos x)$. It is permissible to assume the particle $j$ with tine mass $M$ to be stationary-i.e., $\square=0$. If it is agreed that $\mathrm{v}$ be the velocity, $\delta_{i j}$, of the moving particle w.r.t. the stationary one, then the followling relationship obtains, for the momenta in the binary collision, $M_{r} g=M_{r} \underline{V}_{c m}=m g=m \underline{m}=m \underline{V}^{*}+\mathrm{MV}^{\prime}$, where post-collisional velocities are primed, and the precollisional velocities unprimed. It follows that $m\left(v-V=c m(1-\cos x)=M_{r} g(1-\cos x)\right.$. 


$$
\begin{aligned}
& \phi(\ell)=\int_{0}^{\infty}\left(1-\cos ^{\ell} x\right) g b d b=\left(\frac{e_{1} e_{2}}{M_{r}}\right)^{2} g^{3} \int_{0}^{\infty}\left(1-\cos ^{\ell} x\right) u_{o} d u_{o} \\
& \equiv\left(\frac{e_{1} e_{2}}{M_{r}}\right)^{2} g^{3} A_{\ell}(2),
\end{aligned}
$$

with the integral $A_{\ell}(s) \equiv \int_{0}^{\infty}\left(1-\cos ^{\ell} x\right) u_{0} d u_{0}$ corresponding to Eq. (71); and $s$ denoting the inverse power in the potential law.

The collision frequencles are obtained from the definition

$$
\nu=g n \sigma,
$$

if one substitutes in it the proper expressions for the cross section, $\sigma=Q^{(1)}$, and the flux of test particles, $g \mathrm{n}$. In considering $s=2$ (i.e., the Coulomb interaction) ard using the Debye length $b_{0}$ to define the upper limit, $\mathrm{u}_{\mathrm{Ol}}=\mathrm{b}_{\mathrm{o}} \mathrm{g}^{2} \cdot\left(\mathrm{M}_{\mathrm{r}} / \mathrm{e}_{1} \mathrm{e}_{2}\right)$ for the collision integral, one obtains the quantity*

$$
A_{1}(2) \simeq 2 \ln \left|\frac{3}{2} \frac{(k T)^{3 / 2}}{(\pi n)^{1 / 2} e^{3}}\right|
$$

Hence,

$$
v_{e i}=\frac{2 \pi n e^{4} \ell n \Lambda}{m_{e}^{1 / 2}\left(3 k T_{e}\right)^{3 / 2}},
$$

* The approximation is defined in the text after Eq. (71). Evident 1y, because the approximation is poor for high densities and low temperatures, such values correspond to blanks in Spitzer's 26 Table 5.1, p. 128. Perhaps our equation given in the text after our Eq. (71) might be used. 
where we have substituted $3 \mathrm{kT} T_{e}=M_{r} g^{2}$, and $m_{e} \simeq m_{e} m_{i} /\left(m_{e}+m_{i}\right)=M_{r}$ for the electron-ion encounter. Spitzer's formula (Eq. 5-j1, p. 135 in his book) ${ }^{26}$ agrees with our $\mathrm{Eq} \cdot(76)$, except for the numerical factor, which is $2 \pi$ in our case and $8 / 3(2 \pi)^{1 / 2}$ in spitzer's; so the two results compare like 1:1.06, respectively. Rose and Clarke's collision frequency $[\mathrm{Eq} \cdot(8.20), \mathrm{p} .165$ as corrected by the factor 2 mentioned in the text on p. $\left.166\left(q \cdot v_{\cdot}\right)\right]^{27}$ is the same as our Eq. (76). 


\section{APPEND $1 X C$}

\section{LIOUVILIE'S EQUATION}

The fluid dynamic equations for a system with external forces excrted by the EM fleld represent the fundamental set of balances that obtain in the electric arc. We derive them in a form in which they can be used in the arc model. The Liouville equation expresses the principle of conservation of phase space as an ensemble of particles evolies in time:

$$
\frac{\partial}{\partial t} F(q, p ; t)=\left[\sum_{n=1}^{N}\left[\frac{\partial H}{\partial q_{n}} \frac{\partial}{\partial p_{n}}-\frac{\partial H}{\partial p_{n}} \frac{\partial}{\partial q_{n}}\right]\right] F,
$$

where $F$ is a dynamical function, $q_{n}, p_{n}$ are the canonical coordinates of phase space, and $H$ is the Hamiltonian. The summation is taken over the $N$ degrees of freedom of the system. The r.h.s. in Eq. (1) is the Liouvilitian of F, LF; where L is the operator sum of three components, $\mathrm{L}=\mathrm{L}^{\mathrm{O}}+\mathrm{L}^{\prime}+\mathrm{L}^{\mathrm{F}}$, corresponding to $\mathrm{H}=\mathrm{H}^{\mathrm{O}}+\mathrm{H}^{\circ}+\mathrm{H}^{\mathrm{F}}$ for the free particle, the particle-field interaction, and the field.

For the free particle Hamiltonian $\mathrm{H}^{\mathrm{O}}=\mathrm{p}^{2} / 2 \mathrm{~m}$, we substitute Hamilon"s equations of motion

$$
\frac{\partial H}{\partial q_{n}}=-\dot{p}_{n} ; \quad \frac{\partial H}{\partial p_{n}}=\dot{q}_{n}
$$

In the Poisson Bracket of Jiouville's Eq. (1). To obtain the kinetic equations we simply move the Liouvillian [1.e., the r.h.s. in Eq. (1)] over to the l.h.s. and equate it with source-sink terms, i.e.,

$$
\frac{\partial f}{\partial t}+\bar{v} \nabla f+\stackrel{\nabla}{p} \nabla_{p} f=G-L
$$


We assume constant mass, i.e., $\delta \vec{p}=m \delta \underset{\sim}{\vec{V}}$ and obtain for the laboratory ( $i \cdot e \cdot$, Euler's reference frame)

$$
\frac{\partial f}{\partial t}+\vec{v} \frac{\partial f}{\partial \vec{f}}+\frac{\vec{F}}{m} \frac{\partial f}{\partial \vec{v}}=r \cdot h \cdot s \cdot
$$

where $\vec{F} / m=\vec{v}$. The conservation equations are obtained from Eq. (3) by multiplying it with some velocity function $\vec{p}(\vec{v})$ and integrating the equation over velocity space $\int \mathrm{d} \xi$. It is necessary to do the integration, because a PDE with respect to time and space and not w.r.t. velocity is desired. We thus define the mean values

$$
\begin{array}{lr}
\text { average nurıer density } & n(\vec{r} ; t)=\int d \vec{v} f(\vec{r}, \vec{v} ; t) \\
\text { average dynamical function } & \vec{B}(\vec{r} ; t)=\frac{1}{n} \int d \vec{v} B f(\vec{r}, \vec{v} ; t),
\end{array}
$$

where $f$ is the reduced one-particle distribution function. It is reduced because the system consists of $\mathrm{N}$ distribution functions for $N$ Identical particles which may, for symmetry reasons, be described by the distribution function of just one, arbftrarily selected, particle. Because we have a mixture of species, we need a kinetic equation for each species. The equations are correlated by the r.h.s., where the correlation functions are the collision integrals.

Because no tensors appear in this appendix, we have used the diacritical mark + to denote the vectors. 
I am grateful to H. H. Woodson, Chairman of the electrical engineering department, W. F. Weldon, Director of the Center for Electromechanics at Austin; as well as to H. Dreicer, Director of the magnetic confinement fuston division, and $J$. D. Rogers, Group leader of the energy storage group at Los Alamos, for making this work possible as doctoral research during my sabbatical year at Austin. I benefitted from many discussions with the members of my Supervising Committee, W. C. Duesterhoeft, Jr., Lothar Frommold, E. J. Powers, Jr., R. M. Walser, P. Wildi, H. H. Woodson at Austin, and J. Marshall at Los Alamos. I derlved particular benefit from Frommold's reviews and his, as well as Marshall's, intuic n on the important aspects of this work. I wish to thank Harvey A. Rose and I. R. Lindemuth at Los Alamos: Following a discussion with Rose, I rewrote Chap. 3, in changing it to a more appropriate resolving method for the kinetic equation. Lindemuth offered a review of his two-dimensfonal calculations on the evolution of magnetohydrodynamic turbulence, which enhances thermal conduction in the plasma. The need for such an enhancement mechanism is implied by our cwn results.

I thank the U. S. Department of Energy and the Texas Atomic Energy Research Foundation for the funding of this project.

\section{H. F. V.}

The University of Texas at Austin

Austin, Texas

April 1980. 


\section{REFERENCES}

1. H. G. Thiel, "Zusammensetzung und Transportkoeffizienten von Plasmen mit unterschiedlicher Elektronen- und Gastemperatur und deren Anwendung bei der Berechng. turbulenzbestimmter Hochleistungboegen," Dissertation Marburg (1970).

2. Eugene P. Gross, "Boulder Lectures on Kinetic Theory," Wesley E. Brittin, "Lectures in Theoretical Physics," Vol. 9C (Gordon and Breach 1967), pp. 171-230.

3. Kurt Juchy, "Neue Methoden in der kinetischen Theorie verduennter Gase," in S. Fluegge, F. Trendelenburg, "Ergebnisse der exakten Naturwiss.," (1964) Vol. 35, pp. 103-294.

4. R. Balescu, "Equilibrium and Nonequilibrium Statistical Mechanics," (Wiley, 1975), Chap. 11.5.

5. R. G. Giovanelli, Phil. Mag. (7), 40, 206 (1949).

6. H. Dreicer, Proc. 2nd Internat. Conf. Peaceful Uses of Atomic Energy, Geneva, 1958, 31, 57.

7. H. Hermann, E. Schade, "Transportfunktionen von Stickstoff bis $26000 \mathrm{~K}, " \mathrm{Z}$. Physik 233, 333-350 (1970).

8. Ta-You Wu, "Kinetic Equatlons of Gases and Plasmas," (Addison Wesley, 1966).

9. Sydney Chapman and T. G. Cowling, "The Mathematical Theory of Nonuniform Gases," 3rd Ed. (Cambridge 1970).

10. J. H. Ferziger and H. G. Kaper, "Mathematical Theory of Transport Processes in Gases"' (North Holland, 1972).

11. R. Jance1, Th. Kahan, "Electrodynamics of Plasmas," Wiley, 1966.

12. Harold Grad, "Principles of the Kinetic Theory of Gases," in S. Fluegge, Handb. d. Physik (Springer 1958), Vol. 12, pp 205-294.

13. Joseph 0. Hirschfelder, Charles F. Curtiss, R. Bryon Bird, "Mc lecular Theory of Gases and Liquids," corrected ed. with notes added (Wiley, 1964).

14. Arnold Sommerfeld, "Thermodynamics and Statistical Mechanics," Lectures on Theoretical Physics (Academic Press, 1956), Vol. 5, Sec. $13 c$. 
15. 0 . Theimer, Z. Naturforsch. 12a, 518 (1957).

16. I. Prigogine, "Extension de léquation de Saha au plasma non-isotherme," Bul1. Classe des Sciences, Académie Royale de Belgique, 5th Ser. 16 53-63 (1940).

17. G. Herzberg, "Molecular Spectra and Molecular Structure," Vol. 1, "Spectra of Diatomic Molecules" (Van Nostrand, 1950).

18. A. G. Engelhardt, A. V. Phelps, C. G. Risk, "Determination of Momentum Transfer and Inelastic Collision Cross Sections for Electrons in Nitrogen, Using Transport Coefficients," Phys. Rev. 135, 6A, 1566-1574 (1964).

19. S. Glasstone, R. Lovberg, "Controlled Thermonuclear Reactions," (D. Van Nostrand, 1960), p. 92 .

20. W. Finkelnburg, H. Maecker, "Elektrische Lichtboegen u. Thermisches Plasma," Handb. d. Physik (Springer 1956), Vol. 22, p. 347.

21. Conrad L. Longmire, "Elementary Plasma Physics," (Interscience, 1963).

22. W. Hermann, E. Schade, "Radiative Energy Balance in Cylindrical Nitrogen Arcs," J. Quant. Spectrosc. Radiat. Transfer 12, 1257-1282 (1972).

23. W. Hermann, U. Kogelschatz, K. Ragaller, E. Schade, "Investigation of a Cylindrical, Axially Blown High Pressure Arc," J. Phys. D. Appl. Phys。 7, 607,619 (1974).

24. Wolfgang Hahn, "Theory and Application of Liapunov's Direct Method," (Prentice-Hall, 1963).

25. M. Jteenbeck, Phys. Zs. 33, 809, ff (1932).

26. L. Spitzer, Jr., "Physics of Fully Ionized Gases," 2nd. Ed. (Interscience, 1967 ).

27. D. J. Rose, M. Clark, Jr., "Plasmas and Controlled Fusion," (MIT Press and J. Wi ley, I 961 ).

28. H. Franke, H. G. Thie1, "Theoretische and diagnostische Untersuchung gasbeblasener, turbulenzbestimmter Schaltlichtboegan," Elektrotechn. Z. ETZ-A 92, 282-302. Sec. 6 (1971).

29. George Schmidl, Physics of High Temperature Plasmas, Eq-(5-104), p. 134 (Academic Press, 1966 ). 
30. I. R. Lindemuth, J. S. Pettibone, J. ¿. Stevens, R. C. Harding, D. M. Kraybi11, L. J. Suter, "Unstable 3ehavior of Hot, Magnetized Plasma in Contact with a Cold wal1," Phys. Fluids 21, 1723-1734 $(1978)$. 